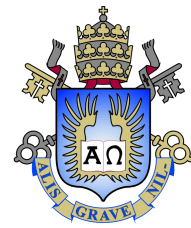

Zhichao Shao

Signal Processing Techniques for Large-scale Multiple-antenna Systems with 1-bit ADCs

Tese de Doutorado

Thesis presented to the Programa de Pós-Graduação em Engenharia Elétrica of PUC-Rio in partial fulfillment of the requirements for the degree of Doutor em Engenharia Elétrica.

Advisor : Prof. Rodrigo Caiado de Lamare

Co-advisor: $\quad$ Prof. Lukas T. N. Landau 


\section{Signal Processing Techniques for Large-scale Multiple-antenna Systems with 1-bit ADCs}

Thesis presented to the Programa de Pós-Graduação em Engenharia Elétrica of PUC-Rio in partial fulfillment of the requirements for the degree of Doutor em Engenharia Elétrica. Approved by the Examination Committee.

Prof. Rodrigo Caiado de Lamare

Advisor

Centro de Estudos em Telecomunicações - PUC-Rio

Prof. Lukas T. N. Landau

Co-advisor

Centro de Estudos em Telecomunicações - PUC-Rio

Prof. Alan Kubrusly

Centro de Estudos em Telecomunicações - PUC-Rio

Prof. Tadeu Ferreira

Universidade Federal Fluminense - UFF

Prof. Vítor Nascimento

Universidade de São Paulo - USP

Prof. Yuriy Zakharov

University of York - York

Prof. João Terêncio Dias

Centro Federal de Educação Tecnológica Celso Suckow da

Fonseca - CEFET/RJ

Rio de Janeiro, June the 24th, 2020 
All rights reserved.

\section{Zhichao Shao}

received his B.Sc. degree in information engineering from Xidian University, China, in 2012, and the Dipl.-Ing. or M.Sc. degree in electrical engineering from Technische Universität Dresden, Germany, in 2016. He works on communications and signal processing.

Bibliographic data

Shao, Zhichao

Signal Processing Techniques for Large-scale Multipleantenna Systems with 1-bit ADCs / Zhichao Shao; advisor: Rodrigo Caiado de Lamare; co-advisor: Lukas T. N. Landau. - Rio de janeiro: PUC-Rio, Departamento de Engenharia Elétrica, 2020.

v., 111 f: il. color. ; $30 \mathrm{~cm}$

Tese (doutorado) - Pontifícia Universidade Católica do Rio de Janeiro, Departamento de Engenharia Elétrica.

Inclui bibliografia

1. Engenharia Elétrica - Teses. 2. Sistemas de múltiplas antenas de larga escala;. 3. CADs de 1 bit;. 4. estimação de canal;. 5. detecção de sinais;. 6. sobreamostragem.. I. Lamare, Rodrigo Caiado de. II. Landau, Lukas T. N.. III. Pontifícia Universidade Católica do Rio de Janeiro. Departamento de Engenharia Elétrica. IV. Título. 


\section{Acknowledgments}

First of all, I would like to thank my supervisor Prof. Rodrigo C. de Lamare and co-supervisor Prof. Lukas T. N. Landau for their encouragement, patience, and guidance during my time as their student. This work would not have been possible without them.

I would like to thank Conselho Nacional de Desenvolvimento Científico e Tecnológico (CNPQ) for funding my research. This study was financed in part by the Coordenação de Aperfeiçoamento de Pessoal de Nível Superior - Brasil (CAPES) Finance Code 001.

I wish to thank my officemates over the years for their friendship and interesting discussions.

Last but not least, I would like to thank my parents for all of their love, encouragements, and on-going support for any of my insane decisions throughout the years. It is my honor to be their son.

This work has been finalized in a period of pandemic of coronavirus in Brazil. Thanks to the professors, my friends, and also the Chinese Consulate continuously caring about my health. I wish you all the best in this hard time. 


\section{Abstract}

Shao, Zhichao; Lamare, Rodrigo Caiado de (Advisor); Landau, Lukas T. N. (Co-Advisor). Signal Processing Techniques for Large-scale Multiple-antenna Systems with 1-bit ADCs. Rio de Janeiro, 2020. 111p. Tese de doutorado - Departamento de Engenharia Elétrica, Pontifícia Universidade Católica do Rio de Janeiro.

Large-scale multiple-antenna systems are a key technique for future wireless communications, which will serve tens of users per base station (BS). In this scenario, one problem faced is the large energy consumption as the number of receive antennas scales up. Recently, low-resolution analogto-digital converters (ADCs) have attracted much attention. Specifically, 1-bit ADCs in the front-end are suitable for such systems due to their low cost and low energy consumption. In this thesis, 1-bit ADCs are applied in three different system designs, which operate at the Nyquist rate and faster than Nyquist rates along with uniform and dynamic strategies.

In the Nyquist-sampling system, low-resolution-aware channel estimation algorithms and a novel iterative detection and decoding scheme are proposed, where short block length low-density parity-check codes are considered for avoiding high latency.

In the faster than Nyquist rates with uniform oversampling system, lowresolution-aware channel estimation and sliding window based detection algorithms are proposed due to their low computational cost and high detection accuracy. Particularly, analytical expressions associated with the Bayesian Cramér-Rao bounds for the oversampled systems are presented. Numerical results are provided to illustrate the performance of the proposed channel estimation algorithms and the derived theoretical bounds.

In the dynamic-oversampling system, two different system designs are devised, namely, sum rate and mean square error based. Three different dimension reduction algorithms are presented and thoroughly investigated. Simulation results show that the systems with the proposed dynamic oversampling outperform the uniformly oversampled system in terms of the computational cost, achievable sum rate and symbol error rate performance.

\section{Keywords}

Large-scale MU-MIMO systems; 1-bit ADCs; channel estimation; signal detection; oversampling. 


\section{Resumo}

Shao, Zhichao; Lamare, Rodrigo Caiado de; Landau, Lukas T. N.. Técnicas de Processamento de Sinais para Sistemas de Múltiplas Antenas de Larga Escala com ADCs de 1-bit. Rio de Janeiro, 2020. 111p. Tese de Doutorado - Departamento de Engenharia Elétrica, Pontifícia Universidade Católica do Rio de Janeiro.

Sistemas de múltiplas antenas de larga escala são técnicas fundamentais para sistemas de comunicação sem fio do futuro, que deverão servir dezenas de usuários por estação rádio-base. Neste contexto, um problema chave é o aumento do consumo de energia à medida que o número de antenas cresce. Recentemente, CADs de baixa resolução têm atraído grande interesse de pesquisa. Em particular, CADs de 1 bit são adequados para sistemas de larga escala devido ao seu baixo custo e consumo de energia. Nesta tese, CADs de 1 bit são usados em três diferentes abordagens de projeto, que operam a taxa de Nyquist e a taxas superiores a taxa de Nyquist com estratégias de amostragem uniforme e dinâmica. Nos sistemas operando a taxa de Nyquist, algoritmos de estimação de canal que exploram o conhecimento da baixa resolução e um novo esquema de detecção e decodificação iterativas são propostos, em que códigos low-density paritycheck de bloco curto são considerados para evitar alta latência.

Nos sistemas operando a taxas superiores a taxa de Nyquist com sobreamostragem uniforme, algoritmos eficientes de estimação de canal e de detecção com janela deslizante com exploração da baixa resolução são propostos. Além disso, são deduzidas expressões analíticas associadas aos limitantes de Cramér-Rao para os sistemas com sobreamostragem. Resultados numéricos ilustram o desempenho dos algoritmos de estimação de canal propostos e existentes e os limitantes teóricos deduzidos.

Nos sistemas operando com sobreamostragem dinâmica, duas abordagens de projeto são desenvolvidas: uma técnica baseada na maximização da soma das taxas e uma técnica baseada na minimização do erro médio quadrático. Em seguida, três algoritmos de redução de dimensão são apresentados e investigados. Resultados de simulações mostram que os sistemas com sobreamostragem dinâmica têm melhor desempenho do que os sistemas com sobreamostragem uniforme em termos de soma das taxas alcançáveis e de taxa de erro de símbolos, enquanto o custo computacional das técnicas examinadas é comparável.

\section{Palavras-chave}

Sistemas de múltiplas antenas de larga escala; CADs de 1 bit; estimação de canal; detecção de sinais; sobreamostragem. 


\section{Table of contents}

$\begin{array}{lll}1 & \text { Introduction } & 16\end{array}$

$\begin{array}{ll}1.1 & \text { Motivation and Prior Works } \\ 1.2 & \text { Contributions }\end{array}$

$\begin{array}{ll}1.2 \text { Contributions } & 17\end{array}$

$\begin{array}{lll}1.3 \text { Outline } & 18\end{array}$

$\begin{array}{lll}1.4 & \text { Notation } & 18\end{array}$

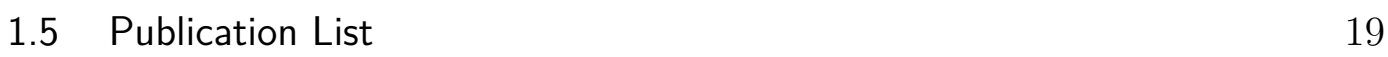

$\begin{array}{ll}\text { 1.5.1 List of Publications } & 19\end{array}$

$\begin{array}{ll}\text { 1.5.2 List of Additional Publications } & 20\end{array}$

2 Technical Background $\quad 21$

2.1 Basics of Large-scale MU-MIMO 21

$\begin{array}{ll}2.1 .1 & \text { MU-MIMO } \\ 2.2 & 21\end{array}$

2.1.2 Sum Rate Capacity 23

2.1.3 Pilot-based Channel Estimation 23

2.1.4 Multiuser Detection 24

2.1.4.1 Optimal Detectors 24

2.1.4.2 Linear Detectors $\quad 25$

2.1.4.3 Nonlinear Detectors 26

$\begin{array}{ll}2.1 .5 & \text { Precoding } \\ 2.1 .5 .1 & 27\end{array}$

$\begin{array}{ll}2.1 .5 .1 & \text { Linear Precoders } \\ 2.1 .5 .2 & 27\end{array}$

2.1.5.2 Nonlinear Precoders 28

2.2 Low-resolution Signal Processing 29

2.2.1 Analog-to-Digital Conversion 29

2.2.2 Oversampling $\quad 32$

2.2.3 Power Consumption 33

2.3 Summary 33

3 Symbol-rate based System Design $\quad 35$

3.1 System model $\quad 35$

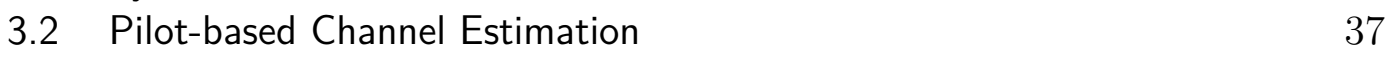

3.2.1 LRA-LMS Channel Estimator 38

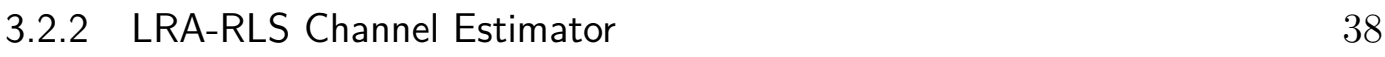

3.2.3 Complexity Analysis 39

3.2.4 Numerical Results $\quad 40$

3.3 Signal Detection $\quad 42$

3.3.1 Proposed LRA-LMMSE Detector 42

3.3.2 Proposed Soft Information Processing and Decoding 44

3.3.2.1 Iterative Decoder 44

3.3.2.2 Quasi-uniform Quantizer 45

3.3.2.3 Adaptive Scaling Factors 45

3.3.3 Numerical Results 46

$\begin{array}{lll}3.4 & \text { Summary } & 47\end{array}$

4 Uniform-oversampling-rate based System Design 49 
4.1 System Model 50

4.2 Noise covariance matrix $\mathbf{C}_{\mathbf{n}} \quad 51$

4.3 Pilot-based Channel Estimation $\quad 52$

4.3.1 Standard LS Channel Estimator 53

4.3.2 LRA-LS Channel Estimator $\quad 54$

4.3.3 LRA-LMMSE Channel Estimator 54

4.3.4 LRA-LMS Channel Estimator 54

4.3.5 Complexity Analysis $\quad 57$

4.3.6 Estimation of $\mathbf{R}_{\mathbf{h}^{\prime}}$

$\begin{array}{lll}\text { 4.3.7 Cramér-Rao Bounds } & 59\end{array}$

4.3.7.1 Bayesian Cramér-Rao Bounds $\quad 59$

4.3.7.2 General Cramér-Rao Bounds $\quad 62$

$\begin{array}{lll}\text { 4.3.8 Numerical Results } & 63\end{array}$

4.3.8.1 $\mathbf{R}_{\mathbf{h}^{\prime}}$ is known at the receiver 64

4.3.8.2 $\mathbf{R}_{\mathbf{h}^{\prime}}$ is unknown at the receiver 66

4.3.8.3 1-bit or b-bit systems 68

$\begin{array}{lll}4.4 & \text { Signal Detection } & 69\end{array}$

4.4.1 Proposed Sliding Window Based Receiver 69

$\begin{array}{lll}\text { 4.4.2 LRA-LMMSE Receive Filter } & 71\end{array}$

4.4.3 LRA-ZF Receive Filter $\quad 72$

$\begin{array}{ll}\text { 4.4.4 Numerical Results } & 72\end{array}$

$\begin{array}{lll}4.5 & \text { Summary } & 73\end{array}$

5 Dynamic-oversampling-rate based System Design $\quad 76$

$\begin{array}{lll}5.1 & \text { System Model } & 77\end{array}$

5.2 System Design with Dynamic Oversampling 79

5.2.1 Sum Rate based System Design 79

5.2.2 Mean Square Error based System Design $\quad 80$

5.3 The Design of the Reduction Matrix $\quad 81$

5.3.1 Generalized Eigenvalue Decomposition 82

5.3.2 Submatrix-level Feature Selection 82

5.3.2.1 Backward Feature Selection 83

5.3.2.2 Restricted Greedy Search $\quad 83$

5.4 Analysis of Proposed Scheme 85

5.4.1 Convergence of the SL-RGS Algorithm 86

5.4.2 Computational Complexity $\quad 87$

5.4.2.1 GEVD vs SL-FS 87

$\begin{array}{ll}\text { 5.4.2.2 Uniform vs Dynamic Oversampling } & 89\end{array}$

$\begin{array}{ll}5.5 \text { Numerical Results } & 90\end{array}$

$\begin{array}{lll}\text { 5.5.1 Sum Rate based System } & 91\end{array}$

5.5.2 MSE based System 91

5.6 Summary 96

6 Conclusions and Future Works $\quad 97$

$\begin{array}{lr}\text { Bibliography } & 99\end{array}$

A Proof of the linear transformation in (3-3) 108 
B Proof of (4-22) 109

$\begin{array}{ll}C \text { Proof of (4-28) } & 110\end{array}$

D Proof of Lemma $1 \quad 111$ 


\section{List of figures}

Figure 2.1 Multiuser MIMO system setup. 22

Figure 2.2 One block of transmission stream at each user 23

Figure 2.3 ADC survey [30] 30

Figure 2.4 Analog to digital conversion process. 30

Figure 2.5 Probability density function of quantization error. 31

Figure 2.6 1-bit analog to digital conversion process. 32

Figure 2.7 1-bit analog to digital conversion with 2-fold oversampling. 33

Figure 2.8 Receiver power consumption as a function of the quantization bits $b$. 34

Figure 3.1 System model of a multiuser multiple-antenna system. 36

Figure 3.2 $N_{t}=4$. Computational complexity comparison between different channel estimators with $\tau=40$ pilot symbols.

Figure 3.3 Normalized MSE comparison between the LRA-LMS channel estimator $(\mu=0.52)$ and other existing estimators.

Figure 3.4 Normalized MSE comparison between the LRA-RLS channel estimator $(\lambda=0.94)$ and other existing estimators.

Figure 3.5 $N_{t}=12$ and $N_{r}=32$. BER performance of IDD schemes under perfect CSI.

Figure 3.6 $N_{t}=9$ and $N_{r}=32$. BER performance of IDD schemes using BLMMSE channel estimation with $\tau=70$ pilot symbols.

Figure 4.1 System model of 1-bit MIMO system with oversampling at the receiver

Figure 4.2 Matrix representation of $\mathbf{G G}^{H}$ with roll-off factor 0.1

Figure 4.3 Matrix representation of $\mathbf{G G}^{H}$ with roll-off factor $0.9 \quad 52$

Figure 4.4 Illustration of the sliding window at each receive antenna when $l_{\text {win }}=3$ and $M=2$, where $l_{\text {win }}$ is the window length representing the number of non-oversampled symbols.

Figure 4.5 Convergence of the LRA-LMS channel estimator with $N_{t}=8$ and $N_{r}=64$ at $\mathrm{SNR}=20 \mathrm{~dB}$.

Figure 4.6 Computational complexity comparison among different channel estimators in an oversampled system $M=3$ with $\tau=20, l_{\text {win }}=3$ and $N_{t}=8$.

Figure 4.7 Normalized MSE comparisons of different channel estimators with known $\mathbf{R}_{\mathbf{h}^{\prime}}$ and $|\rho|=0$

Figure 4.8 Normalized MSE comparisons of different channel estimators with known $\mathbf{R}_{\mathbf{h}^{\prime}}$ and $|\rho|=0.75$

Figure 4.9 SER comparisons of different oversampling factors for the LRA-LMMSE channel estimator with known $\mathbf{R}_{\mathbf{h}^{\prime}}$.

Figure 4.10 Normalized MSE comparisons between LRA-LMMSE and simplified LMMSE [67].

Figure 4.11 Normalized MSE comparisons of different channel estimators with adaptively estimated $\hat{\mathbf{R}}_{\mathbf{h}^{\prime}}$. 
Figure 4.12 Normalized MSE comparisons of different oversampling factors for the LRA-LS channel estimator with estimated $\hat{\mathbf{R}}_{\mathbf{h}^{\prime}}$.

Figure 4.13 Normalized MSE comparisons of LMMSE channel estimator with known $\mathbf{R}_{\mathbf{h}^{\prime}}$ under uncorrelated channel $(|\rho|=0)$.

Figure 4.14 Illustration of one block of oversampled signal at the receiver.

Figure 4.15 Illustration of the sliding window at each receive antenna excluding the first and the last window when $l_{\text {win }}=3$ and $M=2.70$

Figure $4.16 N_{t}=12$ and $N_{r}=64$. SER performance comparison with LRA-ZF receiver, where the black line represents the nonoversampled full-resolution system with ZF receive filter.

Figure $4.17 N_{t}=12$ and $N_{r}=64$. SER performance comparison with LRA-LMMSE receiver, where the black line represents the nonoversampled full-resolution system with LMMSE receive filter.

Figure $4.18 N_{t}=12$ and $N_{r}=64$. SER performance comparison among different window lengths, $M=2$ and $\mathrm{SNR}=20 \mathrm{~dB}$.

Figure 5.1 MU-MIMO with 1-bit ADCs and dynamic oversampling at the receiver.

Figure 5.2 Convergence of the proposed SL-RGS to the unrestricted GS algorithm.

Figure 5.3 Comparison of computational complexities among illustrated matrix design algorithms.

Figure 5.4 Computational complexity comparisons between uniform $\left(M=M^{\prime}\right)$ and dynamic oversampling techniques $\left(M^{\prime}=2\right)$.

Figure 5.5 Comparison between uniform and GEVD based dynamic oversampling technique with $M^{\prime}=2$.

Figure 5.6 Comparison between uniform and SL-RGS based dynamic oversampling technique with $M^{\prime}=2$.

Figure 5.7 Comparison between simplified SL-BFS and simplified SL-RGS based dynamic oversampling technique with $M^{\prime}=2$.

Figure 5.8 Comparison between uniform and GEVD based dynamic oversampling technique with $M^{\prime}=2$.

Figure 5.9 Comparison between uniform and GEVD based dynamic oversampling technique with $M^{\prime}=2$.

Figure 5.10 Comparison between uniform and SL-RGS based dynamic oversampling technique with $M^{\prime}=2$.

Figure 5.11 Comparison between uniform and SL-RGS based dynamic oversampling technique with $M^{\prime}=2$.

Figure 5.12 Comparison between simplified SL-BFS and simplified SL-RGS based dynamic oversampling technique with $M^{\prime}=2$. 


\section{List of tables}

Table 3.1 Computational complexity of different channel estimators 39

Table 4.1 Computational complexity of different channel estimators 58

Table 5.1 Computational complexities of the illustrated matrix design algorithms

Table 5.2 Computational complexity by using different oversampling techniques in MSE based system 


\section{List of Abreviations}

ADC - Analog-to-digital converter

AMP - Approximate message passing

AGC - Automatic gain control

BC - Broadcast channel

BER - Bit error rate

BIM - Bayesian information matrix

BLMMSE - Bussgang linear minimum mean squared error

BS - Base station

$\mathrm{CN}$ - Check node

CRB - Cramér-Rao bounds

CSI - Channel state information

DSNR - Decreasing signal-to-noise ratio

DPC - Dirty paper coding

EM - Expectation-maximization

ENOB - Effective number of bits

FDD - Frequency division duplexing

FOM - Figure of merit

GEVD - Generalized eigenvalue decomposition

GSNR - Greatest signal-to-noise ratio

IC - Interference cancellation

IDD - Iterative detection and decoding

IHT - Iterative hard thresholding

IID - Independent and identically distributed

IMSE - Increasing mean-square error

ISI - Inter-symbol-interference

LDPC - Low-density parity-check

LLR - Log likelihood ratio

LMS - Least mean squares

LMSE - Least mean-square error

LNA - Low noise amplifier

LO - Local oscillator

LRA - Low-resolution aware

LS - Least squares 
LTE - Like long-term evolution

MAC - Multiple access channel

MAP - Maximum a posterior probability

MF - Matched filter

ML - Maximum likelihood

MLD - Maximum-likelihood-detection

mmWave - Millimeter-wave

MMSE - Minimum mean square error

$\mathrm{MRC}$ - Maximal ratio combining

MSE - Mean square error

MUI - Multiuser interference

MU-MIMO - Multiuser multiple-input multiple-output

OFDM - Orthogonal frequency-division multiplexing

PIC - Parallel interference cancellation

QPSK - Quadrature phase shift keying

$\mathrm{RF}$ - Radio-frequency

RLS - Recursive least squares

RRC - Root-raised-cosine

SER - Symbol error rate

SIC - Serial interference cancellation

SINR - Signal-to-interference noise ratio

SL-FS - Submatrix-level feature selection

SL-BFS - Submatrix-level backward feature selection

SL-RGS - Submatrix-level restricted greedy search

SNR - Signal-to-noise ratio

SPA - Sum product algorithm

SQNR - Signal-to-quantization-noise ratio

TDD - Time division duplexing

THP - Tomlinson-Harashima precoder

UE - User equipment

V-BLAST - Vertical-Bell laboratory layered space time

$\mathrm{VN}$ - Variable node

$\mathrm{VP}$ - Vector perturbation

ZF - Zero forcing 


\section{Introduction}

This chapter first presents the research background and the motivations of this thesis. The main contributions and the structure of this thesis are then provided to readers to access the current state of the art. Moreover, some basic notations used throughout the thesis are introduced. The last section makes a list of publications during the period of working on this thesis.

\section{1}

\section{Motivation and Prior Works}

Multiuser multiple-input multiple-output (MU-MIMO) is currently being used in many wireless communication systems like long-term evolution (LTE), which allows for a small number of antennas at the base station [1]. However, in the last decade wireless applications like mobile phones, laptops and wireless sensors, have experienced an explosive growth and current MU-MIMO systems cannot serve such a large number of users. With large antenna arrays at the base station (BS), large-scale (or massive) MIMO can significantly increase the spectral efficiency, mitigate the propagation loss caused by channel fading, reduce the inter-user-interference and have many other advantages as compared to current systems [2,3]. As such, large-scale MIMO is a key technique for future wireless communication systems [4]. However, many different configurations and deployments need to be reconsidered. For example, by using current high-resolution (8-12 bits) analog-to-digital converters (ADCs) for each element of the antenna arrays at the BS, the hardware cost and the energy consumption may become prohibitively high since the dissipated power scales exponentially with the number of bits [5].

The use of low-cost and low-resolution ADCs is then promoted for large-scale MIMO systems. As one extreme case, 1-bit ADCs can largely reduce the hardware cost and energy consumption of the receiver. Many recent works have studied this area. For instance, the works in [6]- [12] have studied massive MU-MIMO systems with coarsely quantized signals operating over frequency-flat, narrowband channels. The works in [6]- [7] have investigated the uplink sum rate capacity by MU-MIMO systems with 1-bit ADCs at the BS and [8]- [9] have analyzed different precoding techniques 
for the downlink. With regard to channel estimation, the studies in [10]- [11] have proposed the Bussgang linear minimum mean squared error (BLMMSE) and expectation-maximization (EM) based iterative hard thresholding (IHT) channel estimators, respectively. In the context of signal detection used in uplink 1-bit massive MU-MIMO systems, the work in [12] presents a lowcomplexity near maximum-likelihood-detection (near-MLD) algorithm called 1-bit sphere decoding.

Moreover, some prior works have investigated 1-bit ADCs used in wideband communication systems. The works in [13-16] have studied massive MU-MIMO systems with coarsely quantized signals that deploy orthogonal frequency-division multiplexing (OFDM) for wideband communications. Their results show that it is satisfactory to use 1-bit ADCs in wideband massive MU-MIMO-OFDM systems. Furthermore, the studies in [17-19] have discussed some key transceiver design challenges, including channel estimation, signal detection, achievable rates and precoding techniques, in millimeter-Wave (mmWave) massive MIMO systems, which are promising candidates for $5 \mathrm{G}$ cellular systems.

\section{2 \\ Contributions}

The contributions of this thesis are the development of channel estimation and signal detection algorithms used in the 1-bit systems sampling at both Nyquist and oversampling rate.

- In the Nyquist-sampling system, adaptive channel estimators are proposed for achieving low computational cost and high estimation accuracy. Iterative detection and decoding (IDD) schemes are developed for 1-bit quantized systems to mitigate the interference from the other users.

- In the uniform-oversampling system, various channel estimation algorithms are proposed and the corresponding computational complexities are analyzed. The fundamental estimation limits are examined by deriving a Bayesian framework and bounds on channel estimation. To reduce the computational complexity caused by the large number of samples due to oversampling, the sliding window based linear detectors are proposed.

- In the dynamic-oversampling system, two different design criteria are proposed and the dimension reduction algorithms are developed to achieve high accuracy and low complexity. 


\section{3}

\section{Outline}

The rest of the thesis is organized as follows:

- Chapter 2 gives some technical background on this thesis. In the first section, basic concepts of multiuser MIMO are presented, which includes sum rate capacity, channel estimation, multiuser detection and precoding technique. In section 2, some related information about low-resolution signal processing is presented, where the signal is processed after the low-resolution ADCs. The concept of oversampling, which increases the signal-to-quantization-noise ratio (SQNR) of the quantizer, and power consumption of the receiver equipped with low-resolution ADCs are also illustrated.

- Chapter 3 presents the pilot-based channel estimation and detection algorithms for 1-bit ADCs systems sampling at Nyquist rate. The comparison of computational complexity between different channel estimators is also described in this chapter. In order to improve signal detection, an IDD scheme is devised for 1-bit system.

- Chapter 4 presents the pilot-based channel estimation and detection algorithms for 1-bit ADCs systems sampling at oversampling rate. Moreover, the channel estimation limits, Bayesian and general Cramér-Rao bounds (CRB), are also derived depending on the availability of knowledge of channel statistics $\mathbf{R}_{\mathbf{h}^{\prime}}$. In order to perform signal detection, sliding window based detectors are illustrated.

- Chapter 5 gives two system designs for the asynchronous 1-bit MIMO system sampling at dynamic oversampling rate. Different dimension reduction algorithms are presented and the complexities are also compared.

- Chapter 6 presents the conclusions of this thesis and suggests directions in which further research could be carried out.

\section{4 \\ Notation}

The following notation is used throughout the thesis. Matrices are in bold capital letters while vectors in bold lowercase. $\mathbf{I}_{n}$ denotes the $n \times n$ identity matrix and $\mathbf{0}_{n}$ is the $n \times 1$ all-zero column vector. Additionally, $\operatorname{diag}(\mathbf{A})$ is a diagonal matrix only containing the diagonal elements of $\mathbf{A}$. The transpose, conjugate transpose and pseudoinverse of $\mathbf{A}$ are represented by $\mathbf{A}^{T}, \mathbf{A}^{H}$ and $\mathbf{A}^{+}$, respectively. $\mathbf{a}^{*}$ denotes the complex conjugate of $\mathbf{a}$ and $[\mathbf{a}]_{k}$ represents the $k$ th element of vector $\mathbf{a} .(\cdot)^{R}$ and $(\cdot)^{I}$ get the real and imaginary part from 
the corresponding vector or matrix, respectively. $\otimes$ is the Kronecker product. Finally, $\operatorname{vec}(\mathbf{A})$ is the vectorized form of $\mathbf{A}$ obtained by stacking its columns and $\operatorname{det}(\mathbf{A})$ is the determinant function. blkdiag $(\cdot)$ is a block matrix such that the main-diagonal blocks are matrices and all off-diagonal blocks are zero matrices. $\operatorname{Tr}(\cdot)$ is denoted by the operation of trace. $\mathbf{x} \sim \mathcal{C N}(\mathbf{a}, \mathbf{B})$ indicates that $\mathbf{x}$ is a complex Gaussian vector with mean $\mathbf{a}$ and covariance matrix $\mathbf{B}$. The expectation and covariance are denoted as $E\{\cdot\}$ and $\operatorname{Cov}\{\cdot\}$, respectively. $\lfloor a\rfloor$ gets the largest integer smaller or equal to a and $\bmod (a, b)$ returns the remainder after division of a by $\mathrm{b}$. While calculating the computational costs, $\mathcal{O}(\cdot)$ represents the big $\mathrm{O}$ notation indicating the number of flops.

\section{5}

\section{Publication List}

\subsection{1}

\section{List of Publications}

During the preparation of this thesis, several parts have been published (or are under review for publication) as articles in peer-reviewed journals or have been presented at international conferences:

Journal papers:

1. Z. Shao, R. C. de Lamare and L. T. N. Landau, Iterative Detection and Decoding for Large-Scale Multiple-Antenna Systems With 1-Bit ADCs, in IEEE Wireless Communications Letters, vol. 7, no. 3, pp. 476-479, June 2018.

2. Z. Shao, L. T. N. Landau and R. C. de Lamare, Channel Estimation for Large-Scale Multiple-Antenna Systems Using 1-Bit ADCs and Oversampling, in IEEE Access, vol. 8, pp. 85243-85256, 2020.

3. Z. Shao, L. T. N. Landau and R. C. de Lamare, Dynamic Oversampling for 1-Bit ADCs in Large-Scale Multiple-Antenna Systems, in IEEE Transactions on Signal Processing, 2020, under review.

Conference papers:

1. Z. Shao, L. T. N. Landau and R. C. de Lamare, Adaptive RLS Channel Estimation and SIC for Large-Scale Antenna Systems with 1-Bit ADCs, WSA 2018; 22nd International ITG Workshop on Smart Antennas, Bochum, Germany, 2018.

2. Z. Shao, L. T. N. Landau and R. C. de Lamare, Sliding Window Based Linear Signal Detection Using 1-Bit Quantization and Oversampling 
for Large-Scale Multiple-Antenna Systems, 2018 IEEE Statistical Signal Processing Workshop (SSP), Freiburg, 2018, pp. 183-187.

3. Z. Shao, R. C. de Lamare and L. T. N. Landau, Adaptive Channel Estimation and Interference Mitigation for Large-Scale MIMO Systems with 1-Bit ADCs, 2018 15th International Symposium on Wireless Communication Systems (ISWCS), Lisbon, 2018.

4. Z. Shao, L. T. N. Landau and R. C. de Lamare, Channel Estimation Using 1-Bit Quantization and Oversampling for Large-scale Multipleantenna Systems, ICASSP 2019 - 2019 IEEE International Conference on Acoustics, Speech and Signal Processing (ICASSP), Brighton, United Kingdom, 2019, pp. 4669-4673.

5. Z. Shao, L. T. N. Landau and R. C. de Lamare, Oversampling based Channel Estimation for 1-bit Large-Scale Multiple-antenna Systems, WSA 2019; 23nd International ITG Workshop on Smart Antennas, Vienna, Austria, 2019.

6. Z. Shao, L. T. N. Landau and R. C. de Lamare, Dynamic oversampling in 1-bit quantized asynchronous large-scale multiple-antenna systems for sustainable IoT networks, ICASSP 2020 - 2020 IEEE International Conference on Acoustics, Speech and Signal Processing (ICASSP), Barcelona, Spain, 2020.

\section{5 .2}

\section{List of Additional Publications}

Several articles which are not directly related to this thesis have been published or submitted while working on this thesis:

1. C. Healy, Z. Shao, R. M. Oliveira, R. C. d. Lamare and L. L. Mendes, Knowledge-aided informed dynamic scheduling for LDPC decoding of short blocks, in IET Communications, vol. 12, no. 9, pp. 1094-1101, 2018.

2. A. B. L. B. Fernandes, Z. Shao, L. Landau and R. C. de Lamare, Comparator Network Aided Detection for MIMO Receivers with 1-bit Quantization, in Asilomar, 2020, submitted. 


\section{2}

\section{Technical Background}

This chapter reviews some basic concepts related to this thesis, which are important for understanding the techniques used in the field and the remaining chapters. For advanced readers, this chapter can be skipped.

The first section briefly introduces topics related to the MU-MIMO systems including sum rate capacity, channel estimation, multiuser detection and precoding techniques. The second section gives some information about the ADCs, which involves quantization theorem, oversampling technique and power consumption.

\section{1}

\section{Basics of Large-scale MU-MIMO}

MIMO stands for multiple-input multiple-output, which is a method for allowing the transmission and reception of more than one data signal simultaneously over the same radio link. This technique has become an essential element of different wireless communication standards including WIFI, 3G and 4G. The standard MU-MIMO networks tend to use small number of antennas (e.g. below ten), whereas the large-scale MIMO, or massive MIMO, are systems that employ an especially high number of antenna arrays (e.g. a few hundred). They have the potential to increase the capacity without requiring more spectrum, mitigate the propagation loss caused by channel fading, be resistant to the interference and have many other advantages [20]. This section will describe a single-cell MU-MIMO system, which has been used for assessing the techniques developed in this thesis.

\subsection{1}

\section{MU-MIMO}

Generally, multiuser MIMO can be generalized into two categories, the MIMO multiple access channel (MAC) and the MIMO broadcast channel (BC). In cellular networks, the MAC, or uplink, models the channel from mobile devices to the base station, and the BC, or downlink, models the channel from the base station to mobile devices. The system model we consider in the following is outlined in Fig. 2.1, where a single BS with $N_{r}$ antenna elements 
communicates with $N_{t}$ single antenna users ${ }^{1}$. In large-scale MIMO systems, $N_{r}$ is much larger than $N_{t}$.

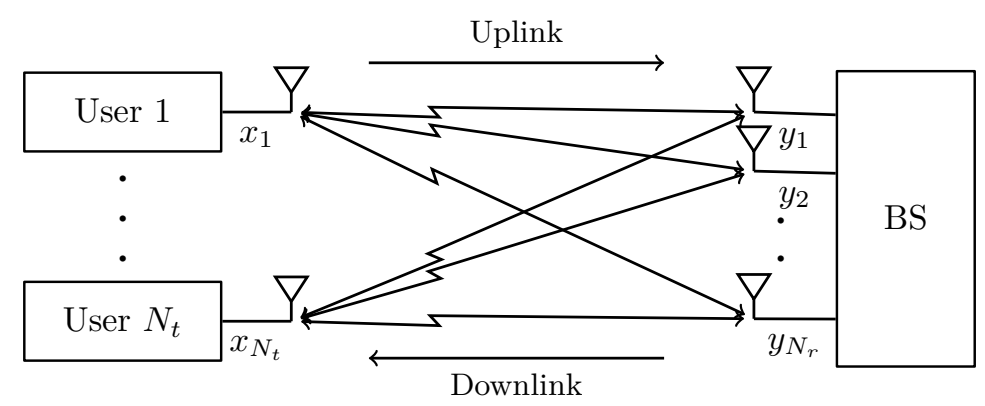

Figure 2.1: Multiuser MIMO system setup.

In the uplink transmission, if the channel between user $n_{t}$ and BS is denoted by $\mathbf{h}_{n_{t}}^{u p} \in \mathbb{C}^{N_{r} \times 1}$, the signal received at the BS is described by

$$
\mathbf{y}^{u p}=\sum_{n_{t}=1}^{N_{t}} \mathbf{h}_{n_{t}}^{u p} x_{n_{t}}+\mathbf{n}^{u p}=\mathbf{H}^{u p} \mathbf{X}+\mathbf{n}^{u p},
$$

where $\mathbf{x}=\left[x_{1}, x_{2}, \ldots, x_{N_{t}}\right]^{T}$. The vector $\mathbf{n}^{u p}$ is the thermal noise at the BS with the size of $N_{r} \times 1$ and $\mathbf{H}^{u p}=\left[\mathbf{h}_{1}^{u p}, \mathbf{h}_{2}^{u p}, \cdots, \mathbf{h}_{n_{t}}^{u p}\right]$ has the size of $N_{r} \times N_{t}$.

On the other hand, the signal received at the $n_{t}$ th user in the downlink is

$$
y_{n_{t}}^{\text {down }}=\mathbf{h}_{n_{t}}^{\text {down }} \mathbf{x}+n_{n_{t}}^{\text {down }}
$$

where $n_{n_{t}}^{\text {down }}$ is the thermal noise at the user $n_{t}$ and the total received signal at the users is

$$
\mathbf{y}^{\text {down }}=\mathbf{H}^{\text {down }} \mathbf{x}+\mathbf{n}^{\text {down }}
$$

where $\mathbf{H}^{\text {down }}=\left[\mathbf{h}_{1}^{\text {down }}, \mathbf{h}_{2}^{\text {down }}, \cdots, \mathbf{h}_{n_{t}}^{\text {down }}\right] \in \mathbb{C}^{N_{r} \times N_{t}}$.

Basically, large-scale MIMO systems can be operated into two modes, time division duplexing (TDD) and frequency division duplexing (FDD). In TDD mode, the channel state information (CSI) remains the same for the uplink and downlink channel, called channel reciprocity, while in FDD mode, the uplink and downlink transmission share the same time but differ in frequency spectrum [22]. In this thesis, TDD transmission and perfect reciprocity is assumed. The superscript in the notation is therefore dropped

$$
\begin{gathered}
\mathbf{h}_{n_{t}}^{u p}=\mathbf{h}_{n_{t}}^{\text {down }}=\mathbf{h}_{n_{t}} \\
\mathbf{H}^{u p}=\mathbf{H}^{\text {down }}=\mathbf{H} .
\end{gathered}
$$

${ }^{1}$ Potentially, each user can be equipped with multiple antennas. The single-antenna user is a simplification and common configuration of research in cellular wireless systems [21]. 


\subsection{2}

\section{Sum Rate Capacity}

The channel capacity of a point-to-point MIMO channel is the fundamental limit on reliable communication: the maximum rate of communication for which arbitrarily small error probability can be achieved [21].

For the MIMO MAC channel, each transmitter is assumed to have an independent message for the base station, and thus the capacity is the sum rate of all users. The upper bound of the sum rate is given by [23]

$$
\sum_{n_{t}=1}^{N_{t}} R_{n_{t}} \leq \log _{2} \operatorname{det}\left(\mathbf{I}_{N_{r}}+\mathbf{H C}_{\mathbf{x}} \mathbf{H}^{H} \mathbf{C}_{\mathbf{n}}^{-1}\right),
$$

where $R_{n_{t}}$ is the rate of user $n_{t}$. $\mathbf{C}_{\mathbf{x}}$ and $\mathbf{C}_{\mathbf{n}}$ represent autocorrelation of transmit and noise signal, respectively.

In the MIMO BC channel, the transmitter is assumed to have a different (and independent) message for each of the receivers. A key component in establishing capacity results for the MIMO BC is the duality relationship between the MIMO BC and the MIMO MAC [23]. The duality relationship refers to the fact that the dirty paper rate region of the MIMO $\mathrm{BC}$ is equal to the union of capacity regions of the dual MIMO MAC, where the union is taken over all individual power constraints and then summation is equal to the power constraint in MIMO BC [24].

\subsection{3}

\section{Pilot-based Channel Estimation}

In the TDD mode, the BS relies on good uplink channel knowledge to recover the data symbols transmitted from each user. A common technique for channel estimation is to let the users transmit a known sequence of symbols ( $\tau$ pilots) and evaluate the effect of the channel on these symbols at the BS, as shown in Fig. 2.2. The mathematical form can be illustrated as

$$
\mathbf{Y}_{p}=\mathbf{H X}_{p}+\mathbf{N}_{p}
$$

where $\mathbf{X}_{p}$ and $\mathbf{Y}_{p}$ has the size of $N_{t} \times \tau, N_{r} \times \tau$, respectively. Vectorizing (2-7) we obtain

$$
\mathbf{y}_{p}=\left(\mathbf{X}_{P}^{T} \otimes \mathbf{I}_{N_{r}}\right) \mathbf{h}+\mathbf{n}_{p}=\tilde{\mathbf{X}}_{P} \mathbf{h}+\mathbf{n}_{p}
$$

where $\mathbf{y}_{p}$ and $\mathbf{h}$ has the size of $N_{r} \tau \times 1$ and $N_{r} N_{t} \times 1$, respectively. In the following, two basic approaches for estimating the channel vector $\mathbf{h}$ are introduced.

\begin{tabular}{|c|c|}
\hline pilot & data \\
\hline
\end{tabular}

Figure 2.2: One block of transmission stream at each user 
The least squares (LS) approach seeks to minimize the squared error between the received pilot sequence and its noise-and-interference free version [25] as described by

$$
\begin{aligned}
\hat{\mathbf{h}}_{\mathrm{LS}} & =\underset{\overline{\mathbf{h}}}{\arg \min }\left\|\mathbf{y}_{p}-\tilde{\mathbf{X}}_{P} \overline{\mathbf{h}}\right\|^{2} \\
& =\left(\tilde{\mathbf{X}}_{p}^{H} \tilde{\mathbf{X}}_{p}\right)^{-1} \tilde{\mathbf{X}}_{p}^{H} \mathbf{y}_{p} .
\end{aligned}
$$

The linear minimum mean square error (MMSE) estimator exploits the prior knowledge of covariance matrices to improve the channel estimates, by amplifying the signal from spatial direction of desired terminal and attenuating the interferers [25]. The linear filter is

$$
\begin{aligned}
\mathbf{W}_{\mathrm{MMSE}} & =\underset{\mathbf{W}}{\arg \min } E\left\{\left\|\mathbf{h}-\mathbf{W}_{\mathbf{y}_{p}}\right\|^{2}\right\} \\
& =\mathbf{C}_{\mathbf{h y}_{p}} \mathbf{C}_{\mathbf{y}_{p}}^{-1},
\end{aligned}
$$

where $\mathbf{C}_{\mathbf{h y}_{p}}$ and $\mathbf{C}_{\mathbf{y}_{p}}$ are defined as $E\left\{\mathbf{h y} \mathbf{y}_{p}^{H}\right\}$ and $E\left\{\mathbf{y}_{p} \mathbf{y}_{p}^{H}\right\}$, respectively. The linear MMSE channel estimator is given by

$$
\hat{\mathbf{h}}_{\mathrm{MMSE}}=\mathbf{C}_{\mathbf{h y}_{p}} \mathbf{C}_{\mathbf{y}_{p}}^{-1} \mathbf{y}_{p} \text {. }
$$

Both estimators have their drawbacks. The MMSE estimator suffers from a high complexity, whereas the LS estimator has a higher MSE.

\subsection{4}

\section{Multiuser Detection}

Multiuser detection is based on the idea of detecting signals of users, and exploiting the detected signal to mitigate their effect on the desired user signal [26]. Generally, besides the optimum maximum likelihood (ML)/maximum a posterior probability (MAP) detectors the other suboptimum multiuser detectors can be characterized into two categories, linear and nonlinear detectors. The former class includes the matched filter (MF), zero-forcing (ZF) receiver and the minimum mean square error (MMSE) receiver; the latter includes interference cancellation (IC), decision feedback receivers and turbo receivers [27].

\subsubsection{1}

\section{Optimal Detectors}

In communication systems, the optimum rule of designing optimal detectors looks for minimizing the error probability based on the observed signals and a given set of hypotheses, which is called the maximum a posteriori (MAP) criterion. When considering the system model of (2-1), the MAP cri- 
terion based MIMO detector is formulated as

$$
\hat{\mathbf{x}}_{\mathrm{MAP}}=\arg \max \operatorname{Pr}(\mathbf{x} \mid \mathbf{y}) .
$$

Using Bayes' rule, the a posteriori probability (APP) can be expressed as

$$
\operatorname{Pr}(\mathbf{x} \mid \mathbf{y})=\frac{p(\mathbf{y} \mid \mathbf{x}) \operatorname{Pr}(\mathbf{x})}{p(\mathbf{y})}
$$

where $\operatorname{Pr}(\mathbf{x})$ is the a priori probability of $\mathbf{x}$, and $p(\mathbf{y} \mid \mathbf{x})$ is the conditional probability density function $(\mathrm{PDF})$ of the observed signal vector $\mathbf{y}$ given $\mathbf{x}$. The MAP criterion can be simplified when each transmitted signal in $\mathbf{x}$ has an identical a priori probability. Furthermore, considering the fact that $p(\mathbf{y})$ is independent of which particular signal is transmitted, the MAP detector becomes equivalent to the ML detector, which can be computed by

$$
\hat{\mathbf{x}}_{\mathrm{ML}}=\arg \max \operatorname{Pr}(\mathbf{y} \mid \mathbf{x})
$$

Therefore, the MAP criterion is usually used in iterative detection and decoding (IDD) aided receivers, where the a priori probabilities of the transmitted symbols, $\operatorname{Pr}(\mathbf{x})$, may be obtained with the aid of a backward-andforward oriented iterative information exchange between the signal detector and the channel decoder. By contrast, the ML criterion is usually used in uncoded systems, where the a priori probabilities of the transmitted symbols cannot be obtained [27].

\subsubsection{2 \\ Linear Detectors}

Similar to linear equalization for elimination of inter-symbol-interference (ISI), linear detectors can be considered as filtering using a matrix filter for elimination of interference. They have the advantage of low complexity, but suffer from a considerable performance loss in comparison to the optimum detector. More explicitly, the decision statistics of linear MIMO detectors may be expressed as

$$
\hat{\mathbf{x}}=\mathbf{T y}
$$

where $\mathbf{T}$ is the linear transformation (or filtering) matrix with the size of $N_{t} \times N_{r}$ to be designed using various criteria. In the literature, the most popular linear detectors are MF, ZF and MMSE detectors [27].

The ZF criterion based MIMO detector is designed to completely remove interuser interference, but does not take noise into account. If the matrix $\mathbf{H}$ satisfies $N_{r}>N_{t}$ and has a full column rank of $N_{t}$, the linear transformation matrix is given by

$$
\mathbf{T}_{Z F}=\left(\mathbf{H}^{H} \mathbf{H}\right)^{-1} \mathbf{H}^{H} .
$$


The linear transformation matrix $\mathbf{T}$ of (2-15) can be designed according to the MMSE criterion, which minimizes the MSE between the actual transmitted data and the detector's outputs after using the linear transformation matrix. To be more specific, $\mathbf{T}$ is obtained by solving the following optimization problem:

$$
\begin{aligned}
\mathbf{T}_{\mathrm{MMSE}} & =\arg \min _{\mathbf{T}} E\left\{\|\mathbf{x}-\mathbf{T} \mathbf{y}\|_{2}^{2}\right\} \\
& =\mathbf{C}_{\mathbf{x y}} \mathbf{C}_{\mathbf{y}}^{-1},
\end{aligned}
$$

where $\mathbf{C}_{\mathbf{x y}}$ and $\mathbf{C}_{\mathbf{y}}$ are defined as $E\left\{\mathbf{x y}{ }^{H}\right\}$ and $E\left\{\mathbf{y} \mathbf{y}^{H}\right\}$, respectively.

The linear ZF detector completely eliminates the interference amongst the multiple inputs, although the noise power has been enhanced. On the other hand, the linear MMSE detector considers both the multiuser interference (MUI) and the noise to jointly minimize the detection error. It achieves a better balance between the MUI elimination and noise enhancement. Hence, at low SNR the linear MMSE detector achieves a better performance.

\subsubsection{3}

\section{Nonlinear Detectors}

Another important class of suboptimum MIMO detectors are the interference cancellation based MIMO detectors, which are nonlinear and generally achieve a better performance than linear MIMO detectors but at the expense of a higher complexity. Some nonlinear MIMO detectors are listed as follows [27]:

- Successive interference cancellation (SIC): In the most popular SIC detectors, a single symbol $x_{n_{t}}$ is detected at a time. While making the detection of other symbol $x_{n_{t}^{\prime}}$, the interference imposed by $x_{n_{t}}$ on the other symbol $x_{n_{t}^{\prime}}$ will be recreated and subtracted as described by

$$
\mathbf{y}_{n_{t}^{\prime}}=\mathbf{y}-\mathbf{H}_{n_{t}} \hat{x}_{n_{t}},
$$

where $\hat{x}_{n_{t}}$ is the estimated symbol and $\mathbf{H}_{n_{t}} \hat{x}_{n_{t}}$ represents the reconstructed interference from the $n_{t}$ th users.

With this mechanism, the ordering criterion that which symbol is detected first is quite important in this scheme. Some ordering criteria include the decreasing signal-to-noise ratio (DSNR) [35], the greatest signal-to-noise ratio (GSNR) [37], the increasing mean-square error (IMSE) criterion [36], and the least mean-square error (LMSE) criterion [38]. Note that the optimal detection order can be achieved by the vertical-Bell laboratory layered space time (V-BLAST) [39] ordering, which is equivalent to the highest signal-to-interference noise ratio (SINR) ordering. However, when the dimension of transmitted symbol 
vector $\mathbf{x}$ is too high, the processing delay of SIC technique will increase largely.

- Parallel interference cancellation (PIC): In the PIC based detectors, all symbols are detected simultaneously. With initial estimate of all symbols, the interference can be regenerated and subtracted from the received signals. This process may be repeated for several iterations. Among them one iteration is described as

$$
\mathbf{y}_{n_{t}^{\prime}}=\mathbf{y}-\sum_{l=1, l \neq n_{t}^{\prime}}^{N_{t}} \mathbf{H}_{l} \hat{x}_{l},
$$

where $\mathbf{H}_{l} \hat{x}_{l}$ is the reconstructed interference from the $l$ th user.

Compared to SIC, PIC has much lower processing delay. In the context of multi-antenna MIMO systems, the PIC detector was studied mainly in $[40,41]$.

- Other common nonlinear detectors include tree-search based MIMO detectors [42], lattice-reduction aided detectors [43,44], probabilistic data association based detectors [45] and semidefinite programming relaxation based detectors [46].

\subsection{5 \\ Precoding}

In MU-MIMO systems, precoding is another important aspect. Before the transmission, the source signals are precoded in order to reduce the performance loss caused by MUI and channel fading. Normally, precoding is performed at the BS during the downlink transmission. The advantage of this method is that the detection procedure at the user equipment (UE) can be simplified, which can reduce the complexity and the power consumption of the UE.

\subsubsection{1}

\section{Linear Precoders}

The classical ways to deal with the interference are the linear processing approaches, which usually achieve reasonable performance with much lower complexity than the nonlinear precoders. The estimation of the transmitted signal $\hat{\mathbf{x}}$ after the receive filter $\mathbf{T}$ is given by

$$
\hat{\mathbf{x}}=\mathbf{T}(\mathbf{H G} \mathbf{x}+\mathbf{n}), \quad \text { s.t. } \quad E\left\{\|\mathbf{G} \mathbf{x}\|_{2}^{2}\right\}=E_{x},
$$

where $E_{x}$ is the transmit power and $\mathbf{G}$ is the linear precoder with the size of $N_{t} \times N_{t}$ to be designed based on different criteria. Similar to the linear 
detectors in subsection 2.1.4.2, linear precoding strategies also include MF, ZF and MMSE criterion [47].

In the ZF precoder, all interference are termed to zero. The precoded transmitted signal in (2-3) will be

$$
\mathbf{G}_{\mathrm{ZF}}=\beta_{Z F} \mathbf{H}^{H} \mathbf{T}^{H}\left(\mathbf{T H} \mathbf{H}^{H} \mathbf{T}^{H}\right)^{-1},
$$

where $\beta_{Z F}$ is a gain factor to make sure that the transmitted signal power after precoding will not be changed.

However, (2-21) has not considered the noise enhancement. In the MMSE precoder design the knowledge of the noise covariance matrix is necessary, which has the form as

$$
\mathbf{G}_{\mathrm{MMSE}}=\beta_{M M S E}\left(\mathbf{H}^{H} \mathbf{T}^{H} \mathbf{T} \mathbf{H}+\frac{\operatorname{Tr}\left(\mathbf{C}_{\mathbf{n}}\right)}{E_{x}} \mathbf{I}_{N_{t}}\right)^{-1} \mathbf{H}^{H} \mathbf{T}^{H},
$$

where $\beta_{M M S E}$ has the same function as $\beta_{Z F}$.

\subsubsection{2}

\section{Nonlinear Precoders}

Apart from the above-mentioned linear precoders, nonlinear precoders can provide the potential to improve the system performance. The design concept is the dirty paper coding (DPC), which shows that any known interference at the transmitter can be subtracted without the penalty of radio resources if the optimal precoding scheme can be applied on the transmit signal [48]. However, the DPC is a highly nonlinear technique and is too complex for practical implementation [23]. Suboptimal approximations of DPC include Tomlinson-Harashima precoder (THP) [49] and vector perturbation (VP) [50].

- THP: it is a pre-equalization technique proposed for channels with ISI. The basic idea is to use the LQ decomposition on the channel matrix $\mathbf{H}$ as

$$
\mathbf{H}=\mathbf{L Q},
$$

where $\mathbf{L}$ is a lower triangular matrix and $\mathbf{Q}$ is a unitary matrix. Basically there are two structures, decentralized and centralized. The only difference is the location of scaling matrix $\mathbf{G}$, whether it is at receivers or transmitters [51]. In decentralized THP the received signal is

$$
\mathbf{y}=\mathbf{G}_{\mathrm{THP}}(\mathbf{H F x}+\mathbf{n}),
$$

whereas in centralized THP the received signal is

$$
\mathbf{y}=\beta\left(\mathbf{H} \frac{1}{\beta} \mathbf{F G}_{\mathrm{THP}} \mathbf{x}+\mathbf{n}\right),
$$


where $\mathbf{F}=\mathbf{Q}^{H}$ and $\mathbf{G}_{\mathrm{THP}}$ is a diagonal matrix. Each diagonal element is the inverse of the diagonal element of the matrix $\mathbf{L}$. The factor $\beta$ is used to impose the power constraint.

- VP: this approach adds additional complex integer vector to the original data vector. With the perturbation, a near optimal performance is achieved. The perturbed data vector is

$$
\mathbf{x}^{\prime}=\mathbf{x}+\tau \ell
$$

where $\tau$ is a positive real number and $\ell$ is a complex integer vector that needs to be decided. Usually, $\tau$ is fixed according to the chosen constellation.

\section{2}

\section{Low-resolution Signal Processing}

In large-scale MU-MIMO systems, the BS is equipped with a large number of antenna arrays. Each receive antenna is connected to a radiofrequency (RF) chain, which mainly consists of two ADCs, low noise amplifier (LNA), mixers, automatic gain control (AGC) and some filters. Among all these components, the power consumption of ADCs dominates the total power of the whole RF chain. The deployment of today's high-speed and highresolution (more than 8 bits) ADCs is unaffordable for the practical use of large-scale MIMO $[28,29]$. The author in [30] has analyzed the affecting factors on the energy consumption of ADCs and proposed the figure of merit (FOM) formula, which is

$$
\mathrm{FOM}_{w}=\frac{\text { Power }}{2^{\text {ENOB }} f_{s}}
$$

where $E N O B$ represents the effective number of bits and $f_{s}$ is the sampling rate. Moreover, the ADC survey made by R. H. Walden is illustrated in Fig. 2.3 , where it can be seen that at high sampling rate (above $300 \mathrm{MHz}$ ) the energy consumption grows largely.

Low-resolution signal processing refers to techniques for processing coarsely quantized (1-3 bits) signals after the ADCs. The benefits of using such low-resolution ADCs are low cost and low energy consumption, which may be a suitable solution for large-scale MIMO systems.

\subsection{1}

\section{Analog-to-Digital Conversion}

In electronics, an ADC converts a continuous-time and continuousamplitude analog signal to a discrete-time and discrete-amplitude digital 


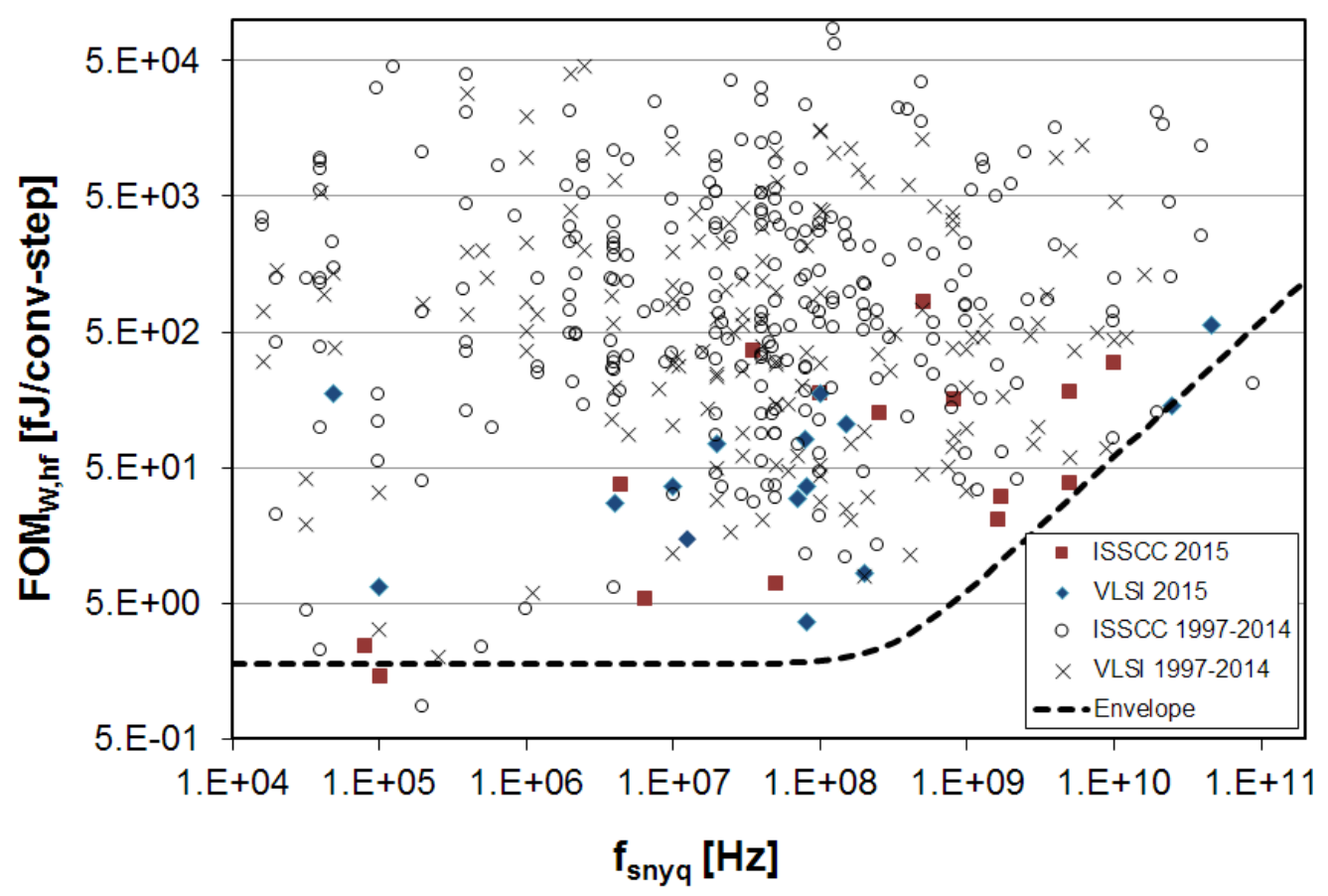

Figure 2.3: ADC survey [30]

signal. Under the control of an external clock, the output of an A/D converter can be stated as

$$
y_{Q}(n)=\mathcal{Q}\{y(n)\}=\mathcal{Q}\left\{y\left(\frac{n T}{M}\right)\right\}, n=1,2, \ldots
$$

where $\mathcal{Q}\{\cdot\}$ represents the quantization function to the corresponding quantization levels and $M$ is the oversampling factor. $T$ is the duration of one symbol. Generally, quantizers can be defined with either uniformly or nonuniformly spaced quantization levels [31]. As one example, Fig. 2.4 shows a typical uniform 3-bit quantizer characteristic, in which the sample values are rounded to the nearest quantization level.

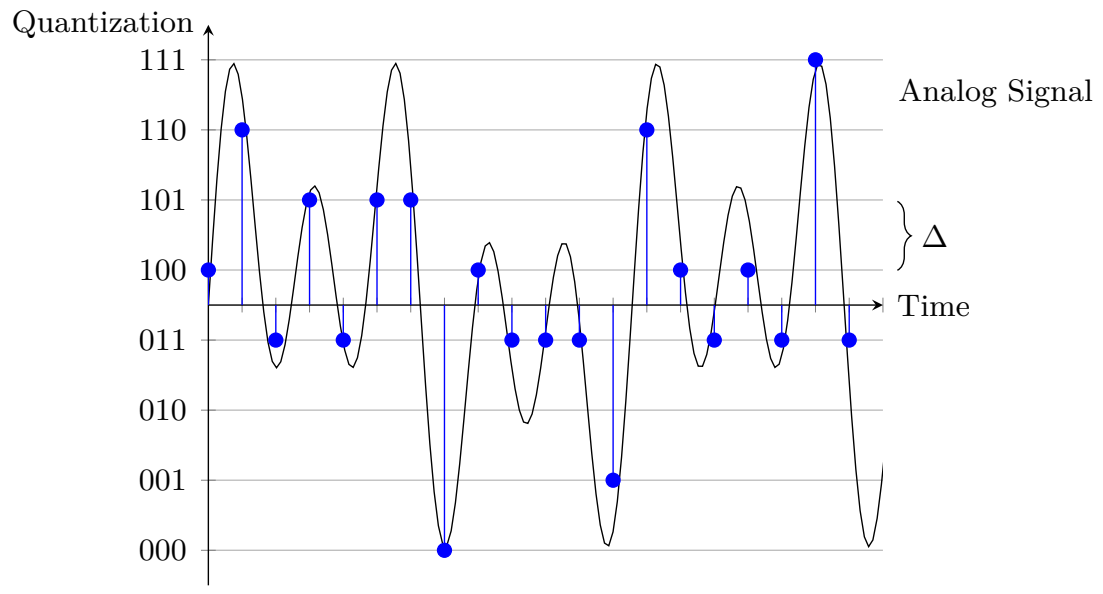

Figure 2.4: Analog to digital conversion process. 
The conversion involves quantization of the input, so it necessarily produces a quantization error, defined as

$$
e[n]=y[n]-y_{Q}[n]
$$

For the example showed in Fig. 2.4, the error is bounded as follows

$$
-\Delta / 2 \leq e[n]<\Delta / 2,
$$

where $\Delta$ is the step-size between two adjacent quantization levels. For small $\Delta$, it is reasonable to assume that $e[n]$ is a random variable uniformly distributed from $-\Delta / 2$ to $\Delta / 2$ [31]. Therefore, the probability density function assumed for the quantization noise is uniformly distributed as shown in Fig. 2.5, and its variance is

$$
\sigma_{e[n]}^{2}=\int_{-\Delta / 2}^{\Delta / 2} e[n]^{2} \frac{1}{\Delta} d e[n]=\frac{\Delta^{2}}{12} .
$$

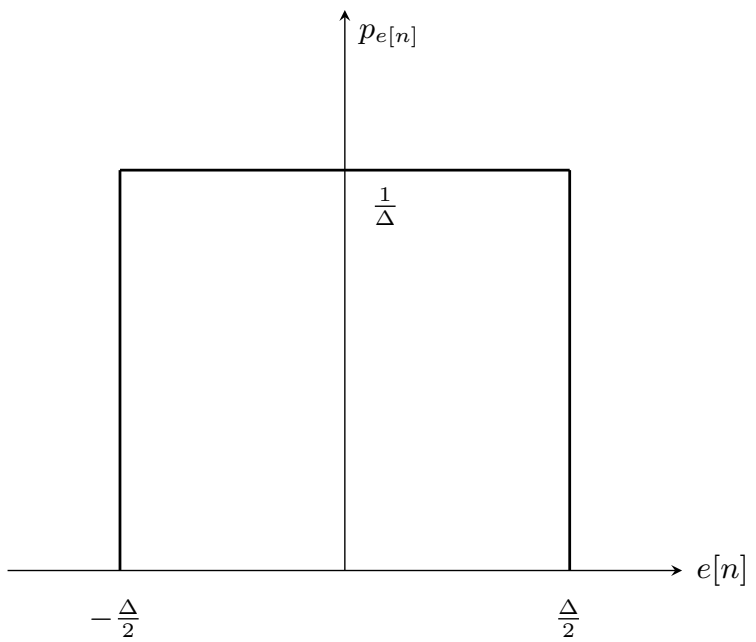

Figure 2.5: Probability density function of quantization error.

As one extreme case, 1-bit ADC has recently attracted much attention by researchers due to its low cost (only constituted by a simple comparator) and low power consumption (will be illustrated in subsection 2.2.3). This thesis mainly concentrates on investigating the signal processing with 1-bit ADCs. In particular, 1-bit ADC can also be termed as a hard-limiter, where the output has only two results, e.g. 1 or -1. Fig. 2.6 shows the 1-bit quantization outputs of the same input signal as in Fig. 2.4, where the quantization error $e[n]$ is extremely large.

Since 1-bit quantization strongly changes the properties of signals, some statistical properties of quantization for Gaussian input signals need to be discussed. For 1-bit quantization and Gaussian inputs, the cross-correlation between the unquantized signal $\mathbf{s}$ with covariance matrix $\mathbf{C}_{\mathbf{s}}$ and its 1-bit quantized signal $\mathbf{s}_{\mathcal{Q}}$ is described by [32] 


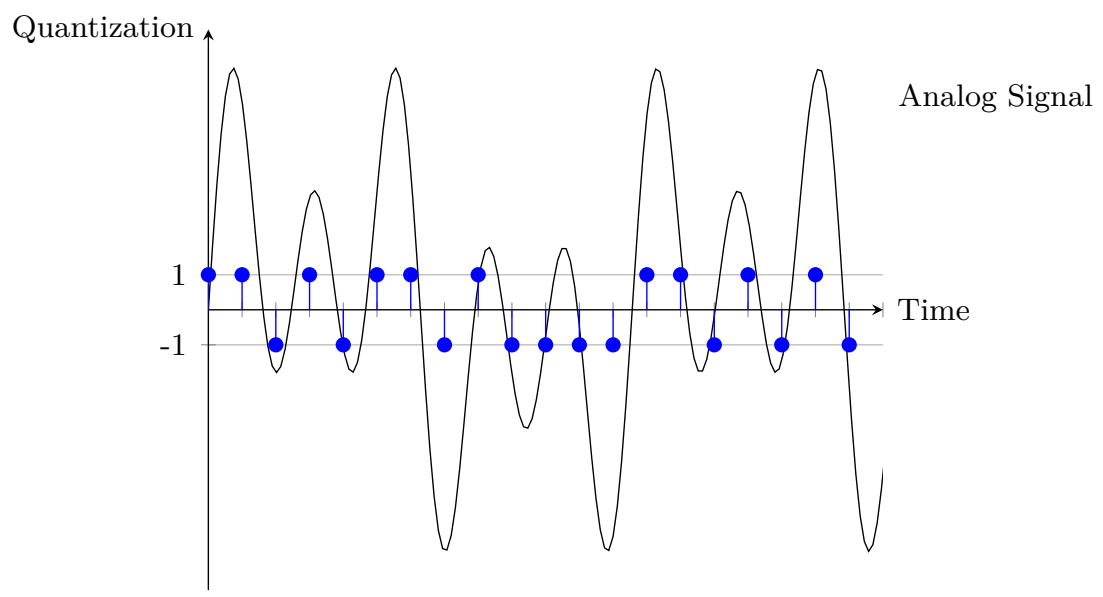

Figure 2.6: 1-bit analog to digital conversion process.

$$
\mathbf{C}_{\mathbf{s}_{\mathcal{Q}} \mathbf{s}}=\sqrt{\frac{2}{\pi}} \mathbf{K C}_{\mathbf{s}} \text {, where } \mathbf{K}=\operatorname{diag}\left(\mathbf{C}_{\mathbf{s}}\right)^{-\frac{1}{2}} .
$$

Furthermore, the covariance matrix of the 1-bit quantized signal $\mathbf{s}_{\mathcal{Q}}$ can be obtained through the arcsin law [33]

$$
\mathbf{C}_{\mathbf{s}_{\mathcal{Q}}}=\frac{2}{\pi}\left(\sin ^{-1}\left(\mathbf{K C}_{\mathbf{s}}^{R} \mathbf{K}\right)+j \sin ^{-1}\left(\mathbf{K C}_{\mathbf{s}}^{I} \mathbf{K}\right)\right) .
$$

\subsection{2}

\section{Oversampling}

In signal processing, oversampling is the process of sampling a signal at a frequency higher than the Nyquist rate, which is defined as twice the bandwidth of the transmission. Theoretically, a bandwidth-limited signal can be perfectly reconstructed if it is sampled at or above the Nyquist rate. Especially, for 1-bit quantization Fig. 2.7 shows an example with 2-fold oversampling of Fig. 2.6, where the red lines represent the samples sampling at the oversampling rate.

In $[31]^{2}$, the authors have illustrated that the noise power of the oversampled signal in the decimated output can be decreased by increasing the oversampling ratio $M$. Since the signal power is independent of $M$, increasing $M$ will increase the signal-to-quantization-noise ratio (SQNR). In other words, oversampling reduces the quantization error caused by the ADCs, expressed by

$$
\sigma_{e[n]}^{2}=\frac{\Delta^{2}}{12 M} .
$$

It can be seen that the quantization error has been reduced to the one- $M$ th of the error in $(2-31)$.

${ }^{2}$ More derivations can be found in the subsection 4.9.1. 


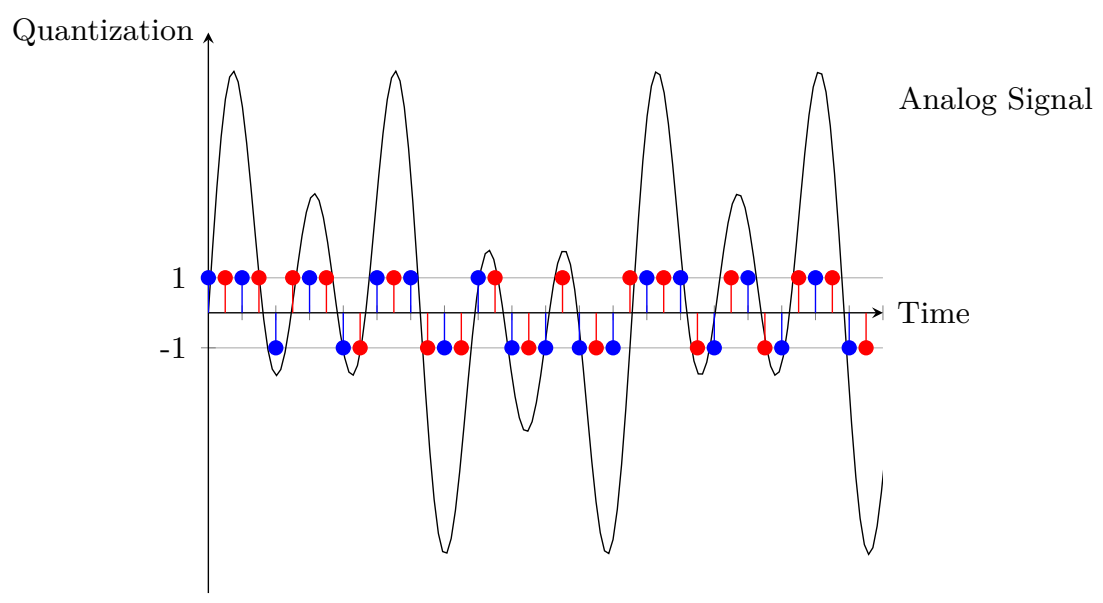

Figure 2.7: 1-bit analog to digital conversion with 2-fold oversampling.

\subsection{3}

\section{Power Consumption}

The advantage of 1-bit ADCs is that they do not require AGC and linear amplifiers, and hence the corresponding radio frequency chains can be implemented with very low cost and power consumption (a few milliwatts) $[7,10,34]$. As an example, Fig. 2.8 shows the total receiver power consumption as a function of the quantization bits $b$. The calculation of the receiver power consumption is based on the work in [52]

$$
P_{\text {total }}=P_{\mathrm{BB}}+P_{\mathrm{LO}}+N_{r}\left(P_{\mathrm{LNA}}+P_{\mathrm{H}}+2 P_{\mathrm{M}}\right)+2 N_{r}\left(c P_{\mathrm{AGC}}+P_{\mathrm{ADC}}\right),
$$

where $P_{\mathrm{BB}}, P_{\mathrm{LO}}, P_{\mathrm{LNA}}, P_{\mathrm{H}}, P_{\mathrm{M}}$ and $P_{\mathrm{AGC}}$ denote the power consumption in the baseband processor, local oscillator (LO), LNA, $\frac{\pi}{2}$ hybrid and LO buffer, mixer and AGC, respectively. The parameter $\mathrm{c}$ is chosen as 0 for the 1-bit system and 1 for b-bit systems $(b>1) . N_{r}=64$. The power consumption of different hardware components is given as $P_{\mathrm{BB}}=200 \mathrm{~mW}, P_{\mathrm{LO}}=22.5 \mathrm{~mW}$, $P_{\mathrm{LNA}}=5.4 \mathrm{~mW}, P_{\mathrm{H}}=3 \mathrm{~mW}, P_{\mathrm{AGC}}=2 \mathrm{~mW}$ and $P_{\mathrm{M}}=0.3 \mathrm{~mW}$. The $P_{\mathrm{ADC}}$ is calculated as

$$
P_{\mathrm{ADC}}=\mathrm{FOM}_{w} \times M f_{n} \times 2^{b},
$$

where $\mathrm{FOM}_{w}$ is $200 \mathrm{fJ} /$ conversion-step at $50 \mathrm{MHz}$ bandwidth and the Nyquist sampling rate $f_{n}$ is $100 \mathrm{MHz}$. From the results, we can see that the 1-bit system consumes much less power than the 2-bit and 3-bit systems in both non-oversampled and oversampled systems.

\section{3}

\section{Summary}

This chapter has reviewed the technical background about this thesis. The basic knowledge about MU-MIMO is illustrated including sum rate 


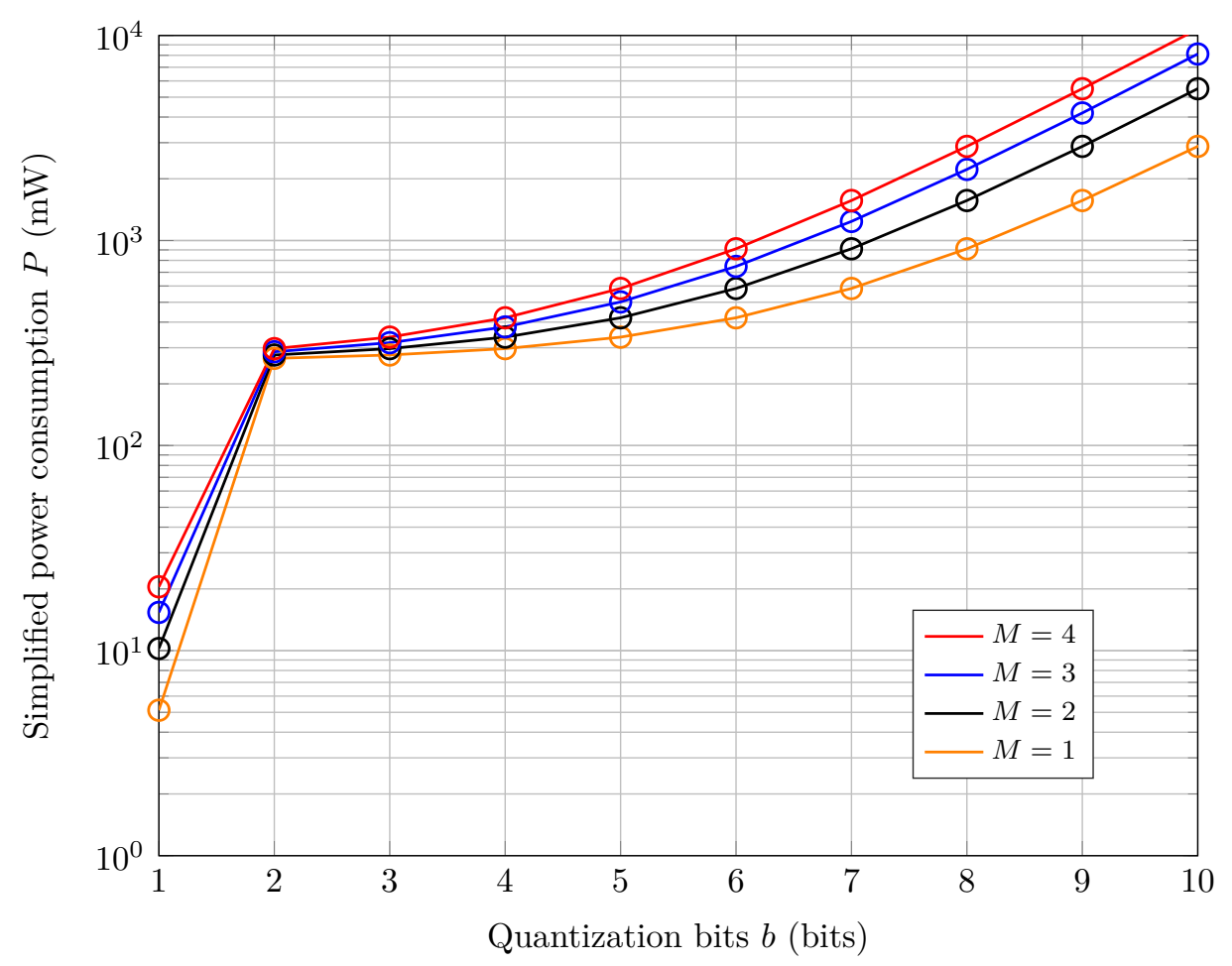

Figure 2.8: Receiver power consumption as a function of the quantization bits $b$.

capacity, channel estimation, multiuser detection and precoding techniques. Moreover, to decrease the power consumption at receiver low-resolution signal processing is equipped with MU-MIMO systems. The function of ADCs and its use with oversampling are also presented and discussed in this chapter. 


\section{3}

\section{Symbol-rate based System Design}

In this chapter, a single-cell large-scale MU-MIMO system with 1-bit ADCs sampling at the symbol or Nyquist rate is considered. This system configuration is used by most of the research. The authors in [18] have examined approximate message passing (AMP) based channel estimation algorithm in millimeter wave MIMO systems with 1-bit quantization. Other channel estimators, such as the Bussgang based LMMSE and the maximum a posteriori probability (MAP), and their performance analysis have been studied in [10] and [53], respectively. The authors in [54] have made an investigation about massive MIMO systems with 1-bit ADCs, which is based on the LS channel estimation, maximal ratio combining (MRC) and ZF detection. An analytical approach to calculate the mutual information for the MRC receiver is also provided. A near ML-type detector and channel estimator are proposed in [55].

The system model is firstly illustrated and two adaptive low-resolution aware channel estimation algorithms are proposed. The computational complexity of existing channel estimators are then compared. In the section of signal detection, an iterative detection and decoding (IDD) scheme with soft interference cancellation and an MMSE-type filter is devised for 1-bit MIMO systems, which is novel compared to prior works.

\section{1}

\section{System model}

The system model is depicted in Fig. 3.1. There are $N_{t}$ single-antenna users and the receiver is equipped with $N_{r}$ antennas, where $N_{r} \gg N_{t}$. The information bits are firstly modulated to $x_{n_{t}}$ according to a given modulation scheme. The transmit symbols $x_{n_{t}}$ have zero-mean and the same energy $E\left\{\left|x_{n_{t}}\right|^{2}\right\}=\sigma_{x}^{2}$. The modulated symbols are then transmitted over blockfading channels with frequency flat. The vector $\mathbf{n} \sim \mathcal{C N}\left(\mathbf{0}_{N_{r}}, \sigma_{n}^{2} \mathbf{I}_{N_{r}}\right)$ contains independent and identically distributed (IID) complex Gaussian random variables with zero mean and variance $\sigma_{n}^{2}$. The received unquantized signal is given by

$$
\mathbf{y}=\mathbf{H} \mathbf{x}+\mathbf{n}=\sum_{n_{t}=1}^{N_{t}} \mathbf{h}_{n_{t}} x_{n_{t}}+\mathbf{n},
$$




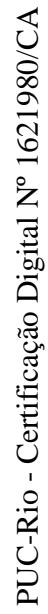

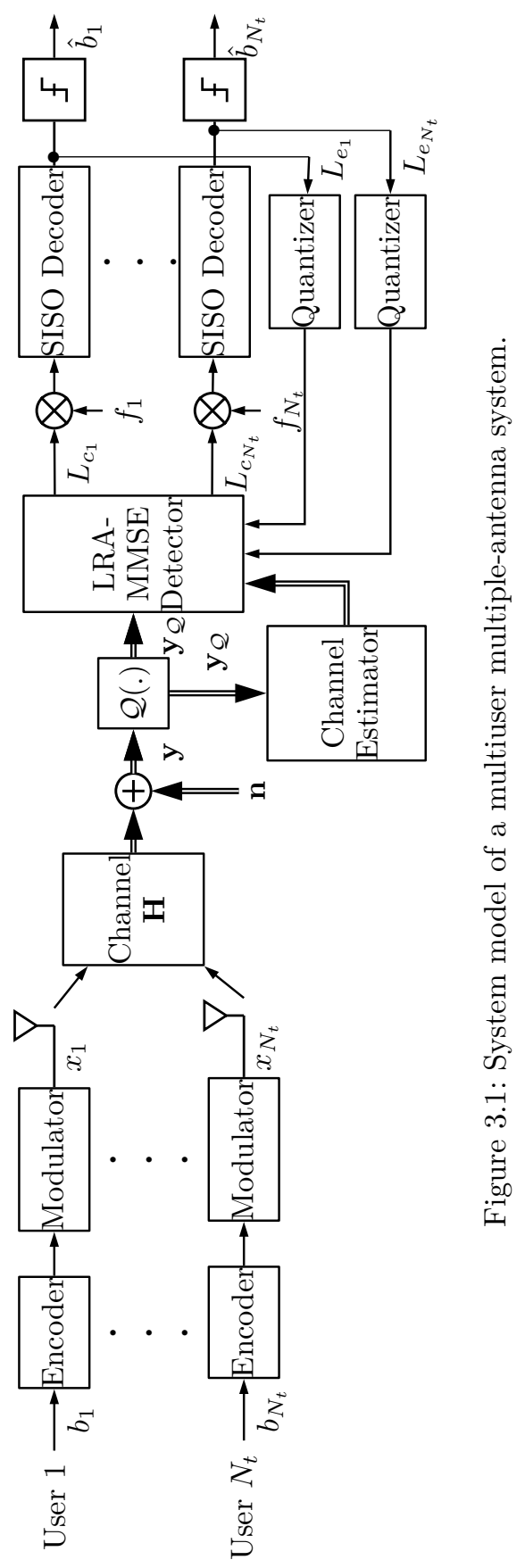


where $\mathbf{h}_{n_{t}} \in \mathbb{C}^{N_{r} \times 1}$ denotes the channel from the user $n_{t}$ to the BS and $\mathbf{H} \in \mathbb{C}^{N_{r} \times N_{t}}$ is the channel matrix. $\mathcal{Q}($.$) represents the 1-bit quantization.$ The real and imaginary parts of the unquantized signal $\mathbf{y}$ are element-wisely quantized to $\left\{ \pm \frac{1}{\sqrt{2}}\right\}$ based on a threshold (e.g. zero). The resulting quantized signal $\mathbf{y}_{\mathcal{Q}}$ is

$$
\mathbf{y}_{\mathcal{Q}}=\mathcal{Q}(\mathbf{y})=\mathcal{Q}(\mathfrak{R}\{\mathbf{y}\})+j \mathcal{Q}(\mathfrak{I}\{\mathbf{y}\}),
$$

where $\mathfrak{R}\{\cdot\}$ and $\mathfrak{I}\{\cdot\}$ get the real and imaginary part, respectively.

Based on (2-32) and (2-33), we can reformulate (3-2) as a statistically equivalent linear system, which is given by

$$
\mathbf{y}_{\mathcal{Q}}=\mathbf{A y}+\mathbf{n}_{q}
$$

where $\mathbf{A}$ is a linear operator chosen independently from $\mathbf{y}$. The vector $\mathbf{n}_{q}$ contains the statistically equivalent quantizer noise with covariance matrix $\mathbf{C}_{\mathbf{n}_{q}}=\mathbf{C}_{\mathbf{y}_{\mathcal{Q}}}-\mathbf{A C}_{\mathbf{y}} \mathbf{A}$. With (2-32) the matrix $\mathbf{A}$ is calculated as

$$
\mathbf{A}=\mathbf{C}_{\mathbf{y y}_{\mathcal{Q}}}^{H} \mathbf{C}_{\mathbf{y}}^{-1}=\sqrt{\frac{2}{\pi}} \operatorname{diag}\left(\mathbf{C}_{\mathbf{y}}\right)^{-\frac{1}{2}}
$$

where $\mathbf{C}_{\mathbf{y}}$ denotes the auto-correlation matrix of $\mathbf{y}$ as

$$
\mathbf{C}_{\mathbf{y}}=E\left\{(\mathbf{H x}+\mathbf{n})(\mathbf{H} \mathbf{x}+\mathbf{n})^{H}\right\}=\sigma_{x}^{2} \mathbf{H} \mathbf{H}^{H}+\sigma_{n}^{2} \mathbf{I}_{N_{r}} .
$$

Proof. See Appendix A.

\section{2}

\section{Pilot-based Channel Estimation}

In the uplink, each transmission packet contains two parts, pilot and data symbols. During the training phase, all $N_{t}$ users simultaneously transmit $\tau$ pilots to the receiver, which yields

$$
\mathbf{Y}_{\mathcal{Q}_{p}}=\mathcal{Q}\left(\mathbf{Y}_{p}\right)=\mathcal{Q}\left(\mathbf{H X}_{p}+\mathbf{N}_{p}\right)
$$

where $\mathbf{X}_{p} \in \mathbb{C}^{N_{t} \times \tau}$ is the pilot matrix.

In large-scale MIMO $N_{r} \gg N_{t}$, the multiplications and divisions involving large matrices, whose dimensions contain $N_{r}$ elements, need to be avoided in order to reduce computational complexity. With this motivation, the channel from $N_{t}$ users to the $n_{r}$ th receive antenna is concentrated, where the received quantized signal is

$$
\mathbf{y}_{\mathcal{Q}_{p}}^{n_{r}}=\mathcal{Q}\left(\mathbf{X}_{p}^{T} \mathbf{h}^{n_{r}}+\mathbf{n}_{p}^{n_{r}}\right)
$$

with $\mathbf{y}_{\mathcal{Q}_{p}}^{n_{r}}=\left[y_{\mathcal{Q}_{p}}^{n_{r}}(1), y_{\mathcal{Q}_{p}}^{n_{r}}(2), \ldots, y_{\mathcal{Q}_{p}}^{n_{r}}(\tau)\right]^{T}$ and $\mathbf{n}_{p}^{n_{r}} \in \mathbb{C}^{\tau \times 1}$. 


\subsection{1}

\section{LRA-LMS Channel Estimator}

Least mean squares (LMS) is the most widely used adaptive algorithm, which has been adopted in various applications like system identification, speech coding and channel equalization [56]. It has the advantage of robust performance and a low cost of implementation. In this subsection, the Bussgang theorem based low-resolution aware (LRA) LMS channel estimator is proposed to compensate for the performance loss due to the quantization of signals to 1 bit.

According to (3-7), the optimization problem that leads to the proposed LRA-LMS channel estimation algorithm can be stated as

$$
\hat{\mathbf{h}}^{n_{r}}(n)=\arg \min _{\mathbf{h}^{n_{r}}(n)} \sum_{n=1}^{\tau}\left|y_{\mathcal{Q}_{p}}^{n_{r}}(n)-A_{p}(n) \mathbf{x}_{p}^{T}(n) \mathbf{h}^{n_{r}}(n)\right|^{2},
$$

where $\mathbf{x}_{p}(n)=\left[x_{1_{p}}(n), x_{2_{p}}(n), \ldots, x_{N_{t_{p}}}(n)\right]^{T}$ and

$$
A_{p}(n)=\mathbf{C}_{\mathbf{y}_{p}^{n_{r}}(n) \mathbf{y}_{\mathcal{Q}_{p}}^{n_{r}}(n)}^{H_{n}} \mathbf{C}_{\mathbf{y}_{p}^{n_{r}}(n)}^{-1}=\sqrt{\frac{2}{\pi}}\left(N_{t} \sigma_{x}^{2}+\sigma_{n}^{2}\right)^{-\frac{1}{2}} .
$$

Taking the partial derivative of the objective function in (3-8) with respect to $\mathbf{h}^{n_{r}}(n)^{H}$, we obtain

$$
\begin{aligned}
& \frac{\partial \sum_{n=1}^{\tau}\left|y_{\mathcal{Q}_{p}}^{n_{r}}(n)-A_{p}(n) \mathbf{x}_{p}^{T}(n) \mathbf{h}^{n_{r}}(n)\right|^{2}}{\partial \mathbf{h}^{n_{r}}(n)^{H}} \\
= & \sum_{n=1}^{\tau}-\left(y_{\mathcal{Q}_{p}}^{n_{r}}(n)-A_{p}(n) \mathbf{x}_{p}^{T}(n) \mathbf{h}^{n_{r}}(n)\right) A_{p}(n) \mathbf{x}_{p}^{*}(n) \\
= & \sum_{n=1}^{\tau}-e(n) A_{p}(n) \mathbf{x}_{p}^{*}(n)
\end{aligned}
$$

The recursion of the proposed LRA-LMS algorithm is

$$
\mathbf{h}^{n_{r}}(n)=\mathbf{h}^{n_{r}}(n-1)+\mu e(n) A_{p}(n) \mathbf{x}_{p}^{*}(n), \quad n=1, \ldots, \tau,
$$

where $\mu$ is the constant stepsize. The initial guess $\mathbf{h}^{n_{r}}(0)$ is an all-zero column vector.

\subsection{2}

\section{LRA-RLS Channel Estimator}

Compared to LMS-based algorithms, the recursive least squares (RLS) algorithms can achieve fast convergence and excellent performance when working in time-varying environments for multiple-antenna systems [57].

According to (3-7), the proposed LRA-RLS algorithm can be derived by solving the following least-squares optimization problem: 


$$
\hat{\mathbf{h}}^{n_{r}}(n)=\arg \min _{\mathbf{h}^{n_{r}}(n)} \sum_{n=1}^{\tau} \lambda^{\tau-n}\left|y_{\mathcal{Q}_{p}}^{n_{r}}(n)-A_{p}(n) \mathbf{x}_{p}^{T}(n) \mathbf{h}^{n_{r}}(n)\right|^{2}+\delta \lambda^{\tau}\left\|\mathbf{h}^{n_{r}}(n)\right\|_{2}^{2},
$$

where $\lambda$ is the forgetting factor and $\delta$ is the regularization factor. The linear operator $A_{p}(n)$ is calculated in (3-9). The LRA-RLS channel estimator is summarized in Algorithm 1.

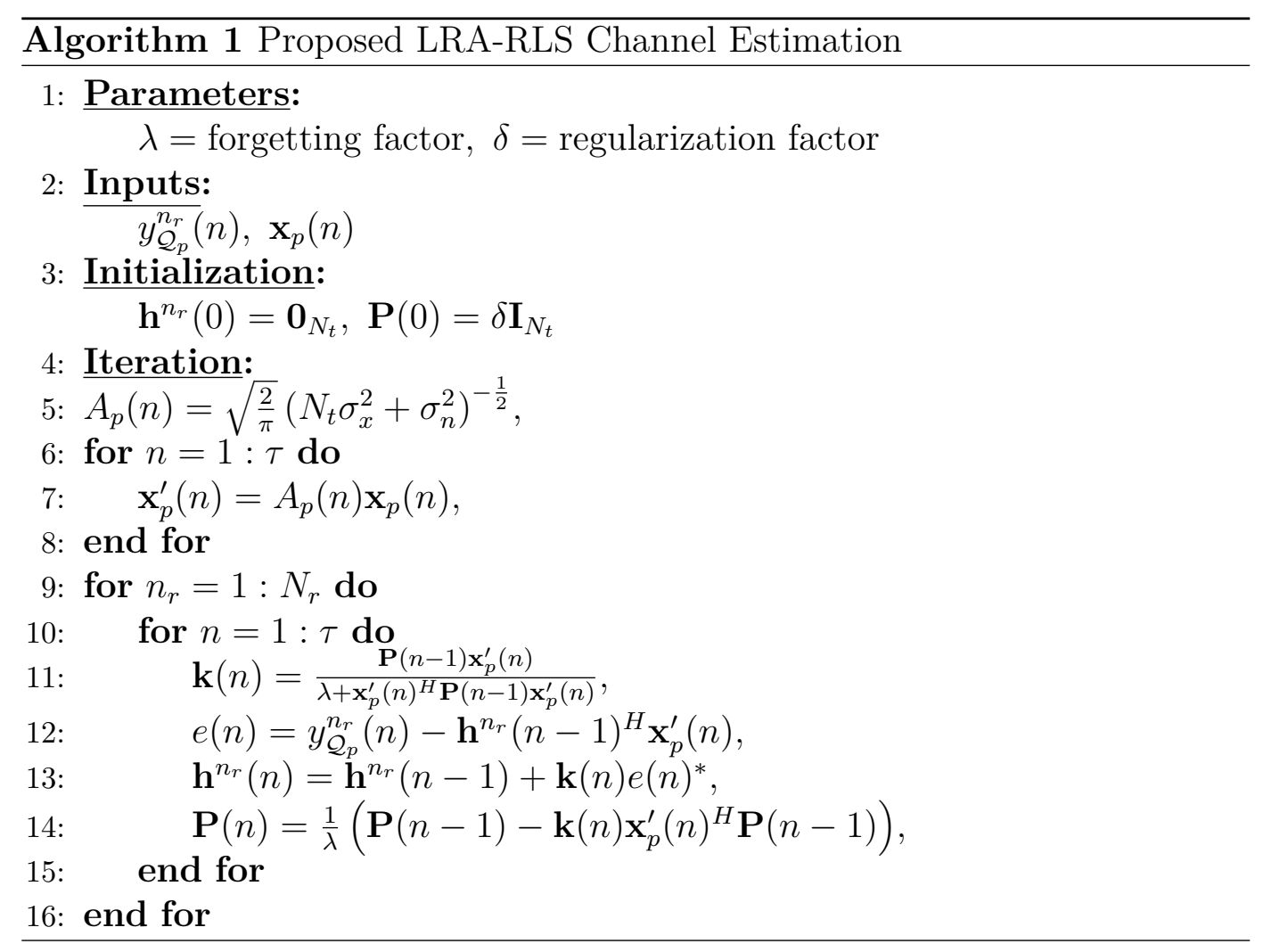

\subsection{3}

\section{Complexity Analysis}

The computational complexity can be calculated as a function of the number of receive antennas $N_{r}$, transmit antennas $N_{t}$ and the length of pilot symbols $\tau$ as depicted in Table 3.1, where other estimation techniques are also listed. Figure 3.2 shows the comparison of computational cost.

\begin{tabular}{|c|c|}
\hline Channel estimator & The order of complexity \\
\hline LS [54] & $\mathcal{O}\left(N_{t}^{3}+\left(\tau+N_{r}\right) N_{t}^{2}+\tau N_{r} N_{t}\right)$ \\
\hline BLMMSE [10] & $\mathcal{O}\left(\left(N_{r} \tau\right)^{3}+\left(N_{r} \tau\right)^{2}\left(36+N_{r} N_{t}\right)+2\left(N_{r} \tau\right)\left(1+N_{r} N_{t}\right)+N_{t} \tau^{2}\right)$ \\
\hline LRA-LMS & $\mathcal{O}\left(4 N_{r} N_{t} \tau+2 N_{r} \tau+3\right)$ \\
\hline LRA-RLS & $\left.\mathcal{O}\left(N_{r} \tau\left(N_{t}^{3}+4 N_{t}^{2}+6 N_{t}+2\right)+\tau N_{t}+3\right)\right)$ \\
\hline
\end{tabular}

Table 3.1: Computational complexity of different channel estimators 


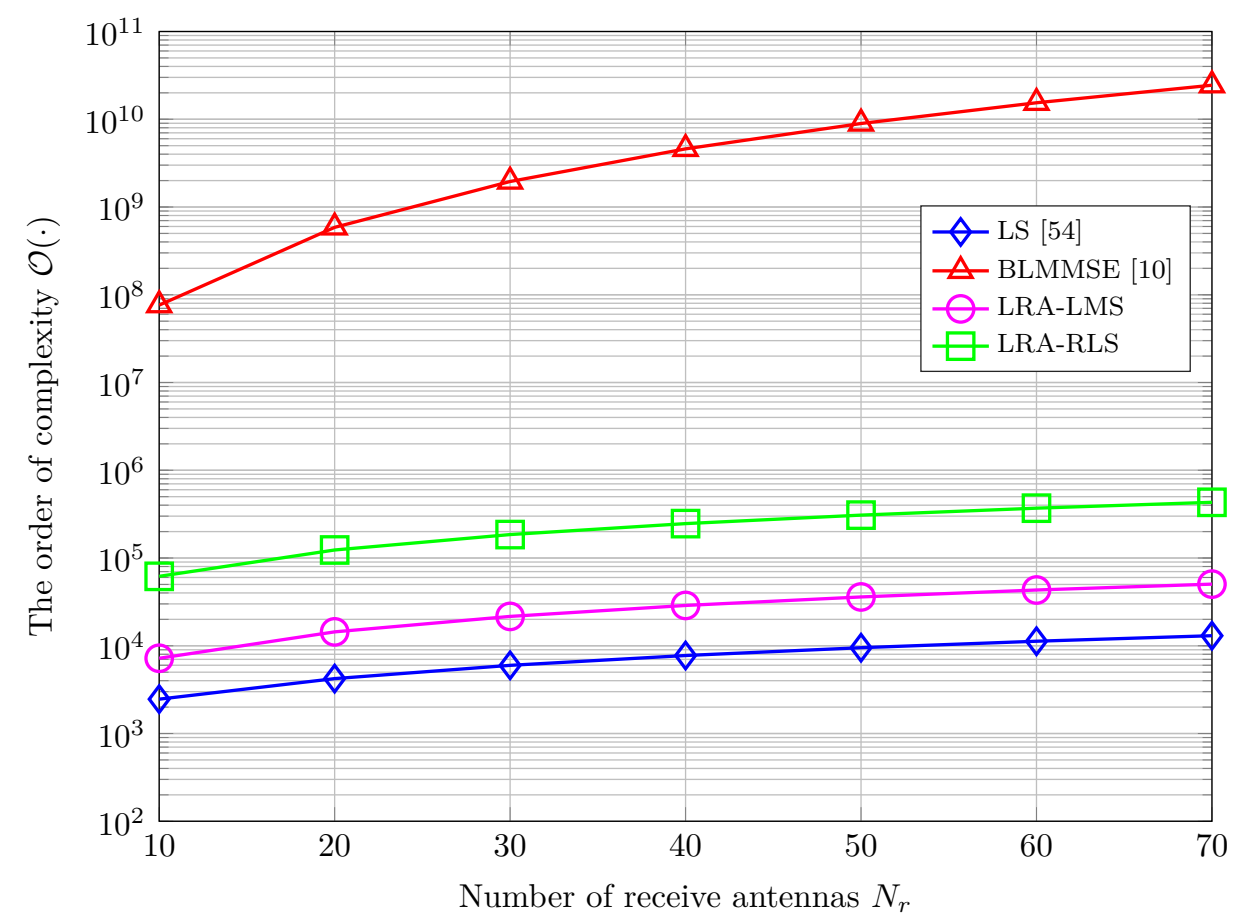

Figure 3.2: $N_{t}=4$. Computational complexity comparison between different channel estimators with $\tau=40$ pilot symbols.

\subsection{4}

\section{Numerical Results}

In this subsection, the proposed adaptive channel estimators are evaluated in terms of the normalized mean squared error (MSE) and are compared with current existing estimators. There are 4 single-antenna users and 16 receive antennas at the $\mathrm{BS}$ in the simulation. The modulation scheme is quadrature phase shift keying $(\mathrm{QPSK})$ and $\mathrm{SNR}=10 \log \left(\frac{N_{t} \sigma_{x}^{2}}{\sigma_{n}^{2}}\right)$. The channel is assumed to experience block fading and the channel matrix is modeled as Gaussian distribution with zero mean and identity covariance matrix. The pilot length at each user is 25. The normalized MSE performance of the LRA-LMS channel estimator and other estimators is illustrated in Fig. 3.3, which shows that the proposed LRA-LMS channel estimator achieves a close performance to that of the BLMMSE algorithm. The normalized MSE performance of the LRA-RLS channel estimator is depicted in Fig. 3.4, which shows that the proposed LRA-RLS channel estimator achieves a close performance to that of the BLMMSE algorithm but with lower complexity. In the LRA-RLS channel estimation phase $\lambda$ is chosen as 0.94 . The value of $\delta$ is not fixed and it increases from $10^{-11}$ to $3 \times 10^{-1}$ while the SNR grows. In both figures, the standard LMS/RLS represents the original LMS/RLS channel estimator without considering the effect of 1-bit quantization. 


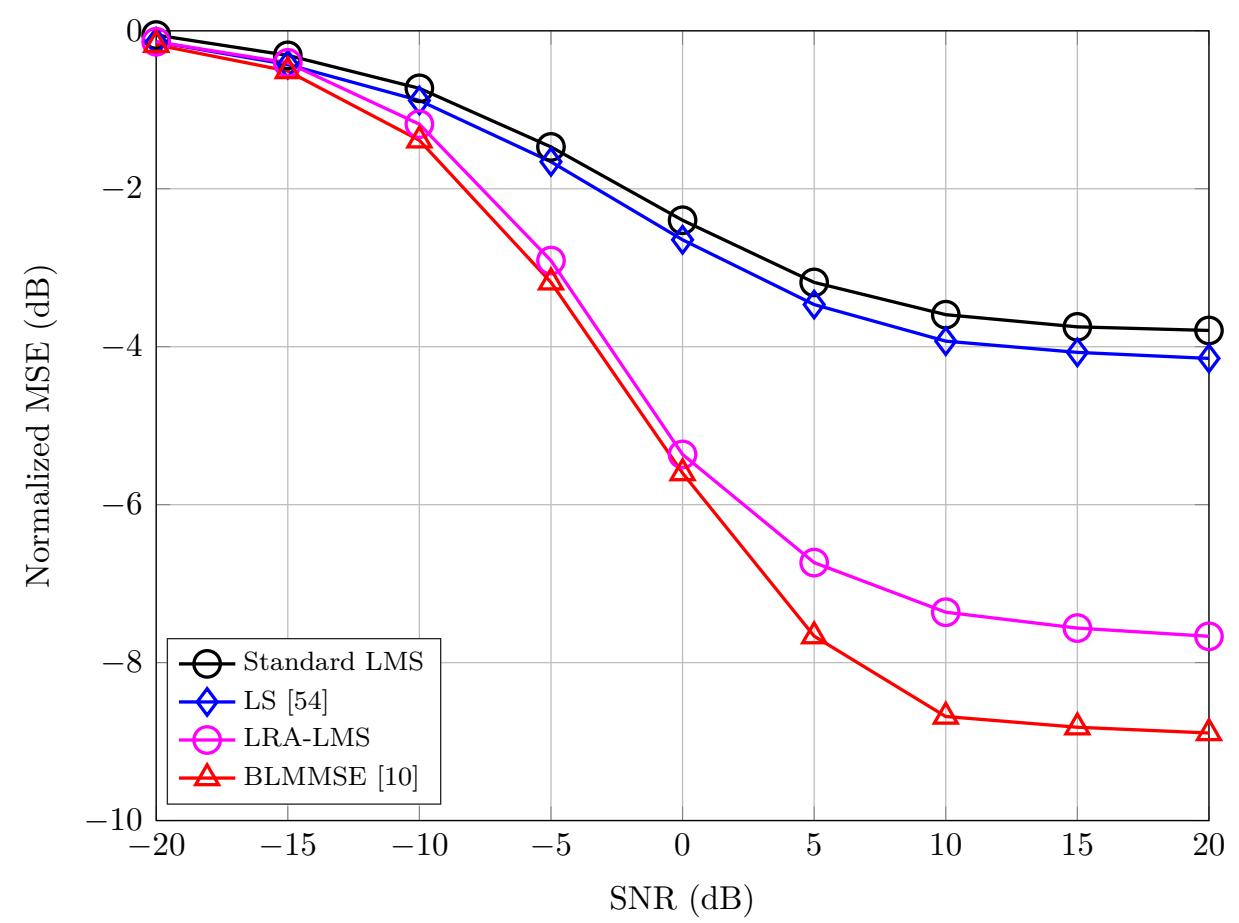

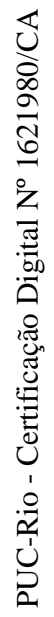

Figure 3.3: Normalized MSE comparison between the LRA-LMS channel estimator $(\mu=$ 0.52 ) and other existing estimators.

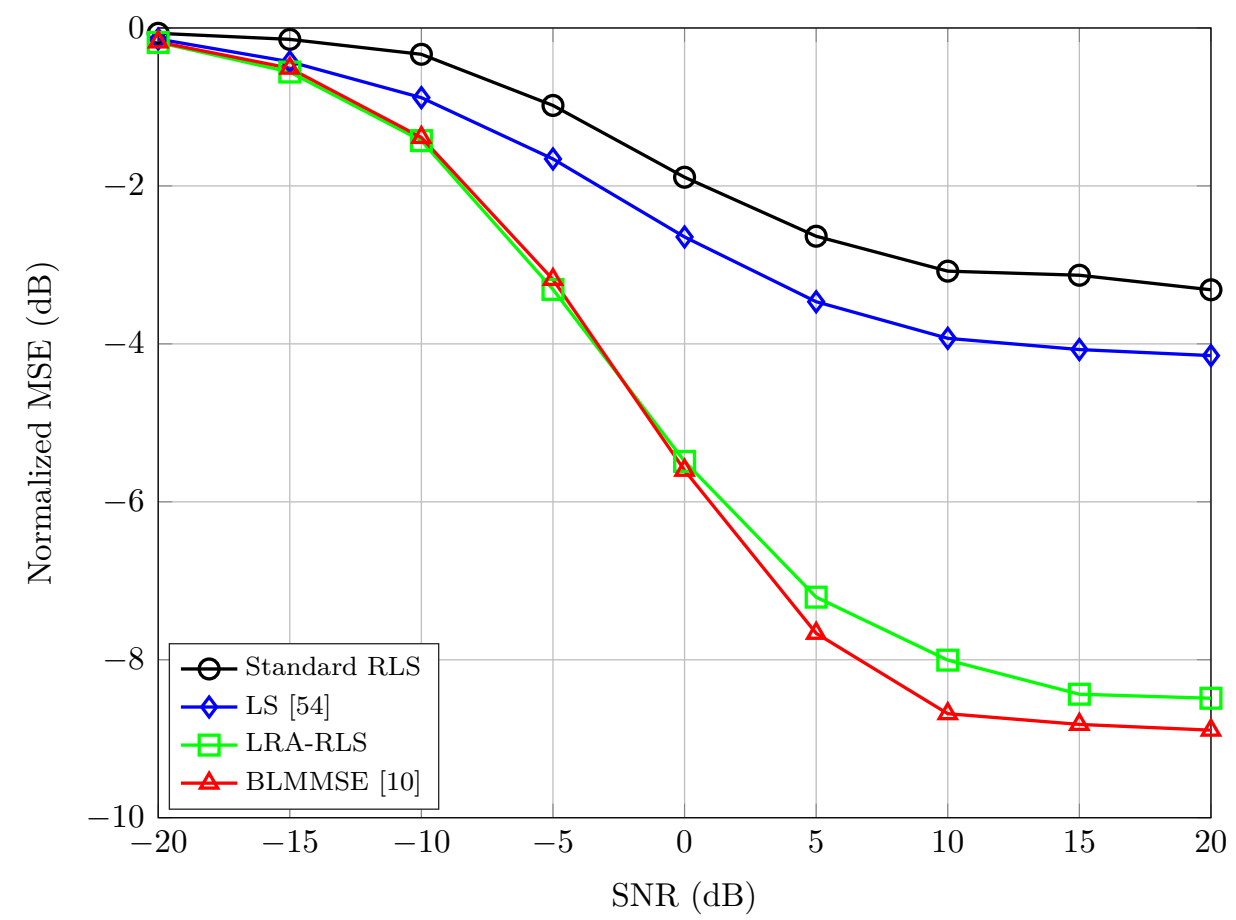

Figure 3.4: Normalized MSE comparison between the LRA-RLS channel estimator $(\lambda=$ 0.94 ) and other existing estimators. 


\section{3}

\section{Signal Detection}

In this section, an IDD scheme is described for 1-bit quantized systems sampling at the Nyquist rate and a low-resolution aware LMMSE (LRALMMSE) receive filter is derived for soft interference mitigation. The key mechanism of the IDD process is the soft information exchange between the detector and the channel decoder, which leads to successive performance improvement [58]. The soft information exchanged often has the form of log likelihood ratio (LLR) of a certain bit. An adaptive decoding approach is developed to combine a quasi-uniform quantization of the passing messages with adjustable scaling factors, which can avoid trapping sets and refine the exchange of LLRs between the detector and the decoder.

\subsection{1}

\section{Proposed LRA-LMMSE Detector}

Inspired by prior works on IDD schemes $[58,59]$, the LRA-LMMSE detector is proposed, which employs a modified LMMSE receive filter and performs soft parallel interference cancellation. The soft estimate of the $n_{t}$ th transmitted symbol is firstly calculated based on the extrinsic LLR $L_{e_{n_{t}}}$ provided by the channel decoder from a previous stage:

$$
\tilde{x}_{n_{t}}=\sum_{x \in \mathcal{A}} x \operatorname{Pr}\left(x_{n_{t}}=x\right)=\sum_{x \in \mathcal{A}} x\left(\prod_{l=1}^{M_{c}}\left[1+\exp \left(-x^{l} L_{e_{n_{t}}}^{l}\right)\right]^{-1}\right),
$$

where $\mathcal{A}$ is the complex constellation set with $2^{M_{c}}$ possible points. The symbol $x^{l}$ corresponds to the value $(+1,-1)$ of the $l$ th bit of symbol $x$. Denote $\tilde{\mathbf{x}}=\left[\tilde{x}_{1}, \ldots, \tilde{x}_{N_{t}}\right]^{T}$ and

$$
\tilde{\mathbf{x}}_{n_{t}}=\tilde{\mathbf{x}}-\tilde{x}_{n_{t}} \mathbf{e}_{n_{t}}
$$

where $\mathbf{e}_{n_{t}}$ is a column vector with all zeros, except that the $n_{t}$ th element is equal to 1 . For each user $n_{t}$, the interference from the other $N_{t}-1$ users is canceled according to

$$
\mathbf{y}_{\mathcal{Q}_{n_{t}}}=\mathbf{y}_{\mathcal{Q}}-\sum_{j=1, j \neq n_{t}}^{N_{t}} \tilde{x}_{j} \mathbf{h}_{j}=\mathbf{y}_{\mathcal{Q}}-\mathbf{H} \tilde{\mathbf{x}}_{n_{t}}
$$

Note that when no prior information is given, $\mathbf{y}_{\mathcal{Q}_{n_{t}}}=\mathbf{y}_{\mathcal{Q}}$. The linear LRALMMSE filter is then applied to $\mathbf{y}_{\mathcal{Q}_{n}}$, to obtain

$$
\hat{x}_{n_{t}}=\mathbf{w}_{n_{t}}^{H} \mathbf{y}_{\mathcal{Q}_{n_{t}}},
$$

where $\mathbf{w}_{n_{t}}$ is chosen to minimize the MSE between the transmitted symbol $x_{n_{t}}$ and the filter output, i.e., 


$$
\begin{aligned}
\mathbf{w}_{n_{t}} & =\arg \min _{\mathbf{w}^{\prime} n_{t}} E\left\{\left\|x_{n_{t}}-\mathbf{w}^{\prime}{ }_{n_{t}} \mathbf{y}_{\mathcal{Q}_{n_{t}}}\right\|^{2}\right\} \\
& =\arg \min _{\mathbf{w}^{\prime} n_{t}}-2 \mathbf{w}^{\prime}{ }_{n_{t}}^{H} \mathbf{c}_{x_{n_{t}} \mathbf{y}_{\mathcal{Q}_{n_{t}}}}+\mathbf{w}^{\prime}{ }_{n_{t}}^{H} \mathbf{C}_{\mathbf{y}_{\mathcal{Q}_{n_{t}}} \mathbf{w}^{\prime}{ }_{n_{t}} .}
\end{aligned}
$$

Differentiating (3-17) with respect to $\mathbf{w}_{n_{t}}^{\prime H}$, the solution of the LRA-LMMSE receive filter is given by

$$
\mathbf{w}_{n_{t}}=\mathbf{C}_{\mathbf{y}_{\mathcal{Q}_{n_{t}}}}^{-1} \mathbf{c}_{x_{n_{t}} \mathbf{y}_{\mathcal{Q}_{n_{t}}}},
$$

where the covariance matrix is

$$
\mathbf{C}_{\mathbf{y}_{\mathcal{Q}_{n_{t}}}}=\mathbf{C}_{\mathbf{y}_{\mathcal{Q}}}-\left(\mathbf{C}_{\mathbf{y}_{\mathcal{Q}} \tilde{\mathbf{x}}_{n_{t}}} \mathbf{H}^{H}\right)^{H}-\mathbf{C}_{\mathbf{y}_{\mathcal{Q}} \tilde{\mathbf{x}}_{n_{t}}} \mathbf{H}^{H}+\mathbf{H C}_{\tilde{\mathbf{x}}_{n_{t}}} \mathbf{H}^{H}
$$

and the cross-correlation vector is

$$
\mathbf{c}_{x_{n_{t}} \mathbf{y}_{\mathcal{Q}_{n_{t}}}}=\sigma_{x}^{2} \sqrt{\frac{2}{\pi}} \mathbf{K h}_{n_{t}}, \quad \text { with } \quad \mathbf{K}=\operatorname{diag}\left(\mathbf{C}_{\mathbf{y}}\right)^{-\frac{1}{2}} .
$$

In (3-19), the covariance matrix of the quantized data vector $\mathbf{y}_{\mathcal{Q}}$ is described by

$$
\mathbf{C}_{\mathbf{y}_{\mathcal{Q}}}=\frac{2}{\pi}\left(\sin ^{-1}\left(\mathbf{K} \Re\left\{\mathbf{C}_{\mathbf{y}}\right\} \mathbf{K}\right)+j \sin ^{-1}\left(\mathbf{K} \mathfrak{I}\left\{\mathbf{C}_{\mathbf{y}}\right\} \mathbf{K}\right)\right),
$$

and the cross-correlation vector between $\mathbf{y}_{\mathcal{Q}}$ and $\tilde{\mathbf{x}}_{n_{t}}$ is

$$
\mathbf{C}_{\mathbf{y}_{\mathcal{Q}} \tilde{\mathbf{x}}_{n_{t}}}=\sqrt{\frac{2}{\pi}} \mathbf{K H C}_{\tilde{\mathbf{x}}_{n_{t}}} .
$$

Note that $\mathbf{C}_{\mathbf{y}}$ is the covariance matrix of the unquantized data vector $\mathbf{y}$, which leads to the following result

$$
\mathbf{C}_{\mathbf{y}}=E\left\{(\mathbf{H x}+\mathbf{n})(\mathbf{H} \mathbf{x}+\mathbf{n})^{H}\right\}=\sigma_{x}^{2} \mathbf{H} \mathbf{H}^{H}+\sigma_{n}^{2} \mathbf{I}_{N_{r}} .
$$

In order to calculate $p\left(\hat{x}_{n_{t}} \mid x\right)$, following [58] the LRA-MMSE filter output can be approximated by a complex Gaussian distribution. The mean and variance of the estimated symbol $\hat{x}_{n_{t}}$, which is conditioned on the transmitted symbol $x$, are given respectively by

$$
\begin{aligned}
& \mu_{n_{t}} \triangleq E\left\{\hat{x}_{n_{t}} \mid x\right\}=\mathbf{w}_{n_{t}}^{H}\left(\mathcal{Q}\left(\mathbf{h}_{n_{t}} x+\mathbf{H} \tilde{\mathbf{x}}_{n_{t}}\right)-\mathbf{H} \tilde{\mathbf{x}}_{n_{t}}\right)
\end{aligned}
$$

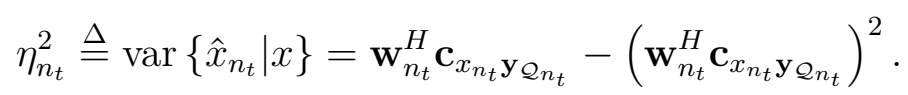

Therefore, the likelihood function can be approximated by

$$
p\left(\hat{x}_{n_{t}} \mid x\right) \simeq \frac{1}{\pi \eta_{n_{t}}^{2}} \exp \left(-\frac{1}{\eta_{n_{t}}^{2}}\left|\hat{x}_{n_{t}}-\mu_{n_{t}}\right|^{2}\right) .
$$

Then the LLR computed by the LRA-MMSE detector for the $l$-th bit $(l \in$ $\left.\left\{1, \ldots, M_{c}\right\}\right)$ of the symbol $\hat{x}_{n_{t}}$ is given by 


$$
\begin{aligned}
L_{c_{n_{t}}}^{l} & =\ln \frac{\operatorname{Pr}\left(b_{n_{t}}^{l}=+1 \mid \hat{x}_{n_{t}}\right)}{\operatorname{Pr}\left(b_{n_{t}}^{l}=-1 \mid \hat{x}_{n_{t}}\right)}-\ln \frac{\operatorname{Pr}\left(b_{n_{t}}^{l}=+1\right)}{\operatorname{Pr}\left(b_{n_{t}}^{l}=-1\right)} \\
& =\ln \frac{\sum_{x \in \mathcal{A}_{l}^{+1}} P\left(\hat{x}_{n_{t}} \mid x\right) \operatorname{Pr}(x)}{\sum_{x \in \mathcal{A}_{l}^{-1}} P\left(\hat{x}_{n_{t}} \mid x\right) \operatorname{Pr}(x)}-L_{e_{n_{t}}}^{l},
\end{aligned}
$$

where $\mathcal{A}_{l}^{+1}$ is the set of hypotheses $x$ for which the $l$-th bit is +1 and $\mathcal{A}_{l}^{-1}$ is similarly defined.

\subsection{2}

\section{Proposed Soft Information Processing and Decoding}

The soft information provided by the LRA-MMSE detector is then fed into a channel decoder that adaptively scales the input LLRs and quasiuniformly quantizes the messages.

\subsubsection{1}

\section{Iterative Decoder}

The decoding method is based on message passing, which iteratively computes the distributions of variables in graph-based models. In the system, the box-plus sum product algorithm (SPA) [60] is used, which is an approximation of SPA decoding. One drawback of SPA is the hyperbolic tangent function, which has numerical saturation problems when computed with finite precision. To avoid such problems, thresholds on the magnitudes of messages must be applied. In the box-plus SPA, the message sent from check node $(\mathrm{CN}) j$ to variable node $(\mathrm{VN}) i$ is

$$
L_{j \rightarrow i}=\boxplus_{i^{\prime} \in N(j) \backslash i} L_{i^{\prime} \rightarrow j},
$$

where $\boxplus$ is the pairwise "box-plus" operator defined as

$$
\begin{aligned}
x \boxplus y & =\ln \left(\frac{1+e^{x+y}}{e^{x}+e^{y}}\right) \\
& =\operatorname{sign}(x) \operatorname{sign}(y) \min (|x|,|y|)+\ln \left(1+e^{-|x+y|}\right)-\ln \left(1+e^{-|x-y|}\right) .
\end{aligned}
$$

The message from $\mathrm{VN} i$ to $\mathrm{CN} j$ is then calculated as

$$
L_{i \rightarrow j}=L_{i}+\sum_{j^{\prime} \in N(i) \backslash j} L_{j^{\prime} \rightarrow i}
$$

where $L_{i}$ is the LLR at $\mathrm{VN} i$. The quantity $j^{\prime} \in N(i) \backslash j$ represents all CNs connected to $\mathrm{VN} i$ except $\mathrm{CN} j$. 


\subsubsection{2}

\section{Quasi-uniform Quantizer}

This quantizer is used both in the decoder and the extrinsic message quantizer to refine or compensate for the effect of 1-bit quantization on the LLRs. The algorithm is based on the quasi-uniform quantization in [61], which represents a compromise between conflicting objectives of retaining fine precision, allowing large dynamic range and implementation complexity. It is a combination of non-uniform and uniform quantization and realized as follows:

$$
Q_{\Delta}^{*}\left(L_{c_{n_{t}}}\right)= \begin{cases}d^{N+1} N \Delta & \text { if } d^{N+1} N \Delta \leq L_{c_{n_{t}}} \\ d^{r} N \Delta & \text { if } d^{r} N \Delta \leq L_{c_{n_{t}}}<d^{r+1} N \Delta, \text { for } 1 \leq r \leq N \\ Q_{\Delta}\left(L_{c_{n_{t}}}\right) & \text { if }-d N \Delta<L_{c_{n_{t}}}<d N \Delta \\ -d^{r} N \Delta & \text { if }-d^{r+1} N \Delta<L_{c_{n_{t}}} \leq-d^{r} N \Delta, \text { for } 1 \leq r \leq N \\ -d^{N+1} N \Delta & \text { if } L_{c_{n_{t}}} \leq-d^{N+1} N \Delta\end{cases}
$$

with

$$
Q_{\Delta}\left(L_{c_{n_{t}}}\right)= \begin{cases}N \Delta & \text { if } N \Delta-\frac{\Delta}{2} \leq L_{c_{n_{t}}} \\ m \Delta & \text { if } m \Delta-\frac{\Delta}{2} \leq L_{c_{n_{t}}}<m \Delta+\frac{\Delta}{2}, \text { for } N>m>0 \\ 0 & \text { if }-\frac{\Delta}{2}<L_{c_{n_{t}}}<\frac{\Delta}{2} \\ m \Delta & \text { if } m \Delta-\frac{\Delta}{2}<L_{c_{n_{t}}} \leq m \Delta+\frac{\Delta}{2}, \text { for }-N<m<0 \\ -N \Delta & \text { if } L_{c_{n_{t}}} \leq-N \Delta+\frac{\Delta}{2}\end{cases}
$$

where $d$ is the growth rate parameter, $\Delta$ is the step size, $N$ is the total number of bits for representing each range and $L_{c_{n_{t}}}$ is the passing message at the $n_{t}$ th decoder.

\subsubsection{3}

\section{Adaptive Scaling Factors}

For improving the decoding performance two scaling factors are deployed, which are obtained offline and online, respectively.

- Offline Scaling Factor: This factor is utilized to correct LLR values used in iterative decoding based on the LLR distribution [62]. In the training phase, data packets are sent to the receiver for obtaining sufficient LLR statistics. For a given SNR, the following steps are carried out:

1) Calculate the probabilities of $\operatorname{Pr}\left(L_{c_{n_{t}}} \mid b_{n_{t}}\right)$ conditioned on transmitted bits $b_{n_{t}}$ through histograms.

2) Obtain $f\left(L_{c_{n_{t}}}\right)=\ln \frac{\operatorname{Pr}\left(L_{c_{n_{t}}} \mid b_{n_{t}}=1\right)}{\operatorname{Pr}\left(L_{c_{n_{t}}} \mid b_{n_{t}}=0\right)}$. 
3) Employ the approximation $f\left(L_{c_{n_{t}}}\right)=\alpha_{n_{t}} L_{c_{n_{t}}}$.

This factor $\alpha_{n_{t}}$ is only applied in the first iteration at the $n_{t}$ th decoder input during the data transmission phase. Moreover, the scaled mean absolute value $\alpha_{n_{t}} \bar{L}_{c_{n_{t}}}$ for each user is stored for calculating the online scaling factor.

- Online Scaling Factor: The factor $f_{n_{t}}$ is calculated at the $n_{t}$ th decoder input in the second iteration and applied for all the iterations except the first iteration. It aims to correct the LLR errors caused by the quantizer. The scaled LLR should be approximated to the scaled LLR in 3). The linear scaling factor is calculated as:

$$
f_{n_{t}}=\alpha_{n_{t}} \bar{L}_{c_{n_{t}}} / \bar{L}_{c_{n_{t}}}^{2 \text { nd iteration }}
$$

where $\bar{L}_{c_{n_{t}}}^{2 \text { nd iteration }}$ is the mean absolute value of LLRs for the $n_{t}$ th user in the second iteration.

\subsection{3}

\section{Numerical Results}

A short length regular low-density parity-check (LDPC) code [63] with block length $n=512$ and rate $1 / 2$ is considered. The modulation scheme is QPSK and the parameters of the quasi-uniform quantizer are $\Delta=0.25$, $d=1.3$ and $N=6$. The channel is assumed to experience block fading and is modeled by IID circularly symmetric complex Gaussian random variables with zero mean and unit variance. The channel matrix is estimated unless otherwise specified through the BLMMSE channel estimator [10]. During training, all $N_{t}$ users simultaneously transmit $\tau$ pilot symbols to the receiver. Recall that the vectorized received signal is described by

$$
\mathbf{y}_{\mathcal{Q}_{p}}=\mathcal{Q}\left(\mathbf{y}_{p}\right)=\mathcal{Q}\left(\tilde{\mathbf{X}}_{p} \mathbf{h}+\mathbf{n}_{p}\right)
$$

where $\tilde{\mathbf{X}}_{p}=\left(\mathbf{X}_{p}^{T} \otimes \mathbf{I}_{N_{r}}\right) \in \mathbb{C}^{N_{r} \tau \times N_{t} N_{r}}$ is the modified pilot matrix. The vector $\mathbf{h} \in \mathbb{C}^{N_{t} N_{r} \times 1}$ is the vectorized channel matrix $\mathbf{H}$. With the assumption $\mathbf{C}_{\mathbf{h}}=\mathbf{I}_{N_{t} N_{r}}$, the estimated channel vector is

$$
\hat{\mathbf{h}}=\left(\mathbf{A}_{p} \tilde{\mathbf{X}}_{p}\right)^{H} \mathbf{C}_{\mathbf{y}_{\mathcal{Q}_{p}}}^{-1} \mathbf{y}_{\mathcal{Q}_{p}}
$$

where $\mathbf{A}_{p}=\sqrt{\frac{2}{\pi}} \operatorname{diag}\left(\mathbf{C}_{\mathbf{y}_{p}}\right)^{-\frac{1}{2}} \cdot \mathbf{C}_{\mathbf{y}_{\mathcal{Q}_{p}}}$ and $\mathbf{C}_{\mathbf{y}_{p}}$ are calculated according to (3-21) and (3-23), respectively.

The bit error rate (BER) performance of IDD schemes under perfect CSI are shown in Fig. 3.5. It can be seen that the proposed LRA-MMSE detector obtains a large gain compared to the traditional MMSE one. Moreover, Fig. 
3.5 also depicts the BER performance of IDD schemes with and without quasi-uniform quantizer and scaling factors, which shows the system has a significant performance gain after 2 iterations. These results also demonstrate that the quantizer and the scaling factors offer extra performance gains. Fig. 3.6 illustrates the system performance using BLMMSE channel estimation, where $\tau=70$ pilot symbols are used in each block.

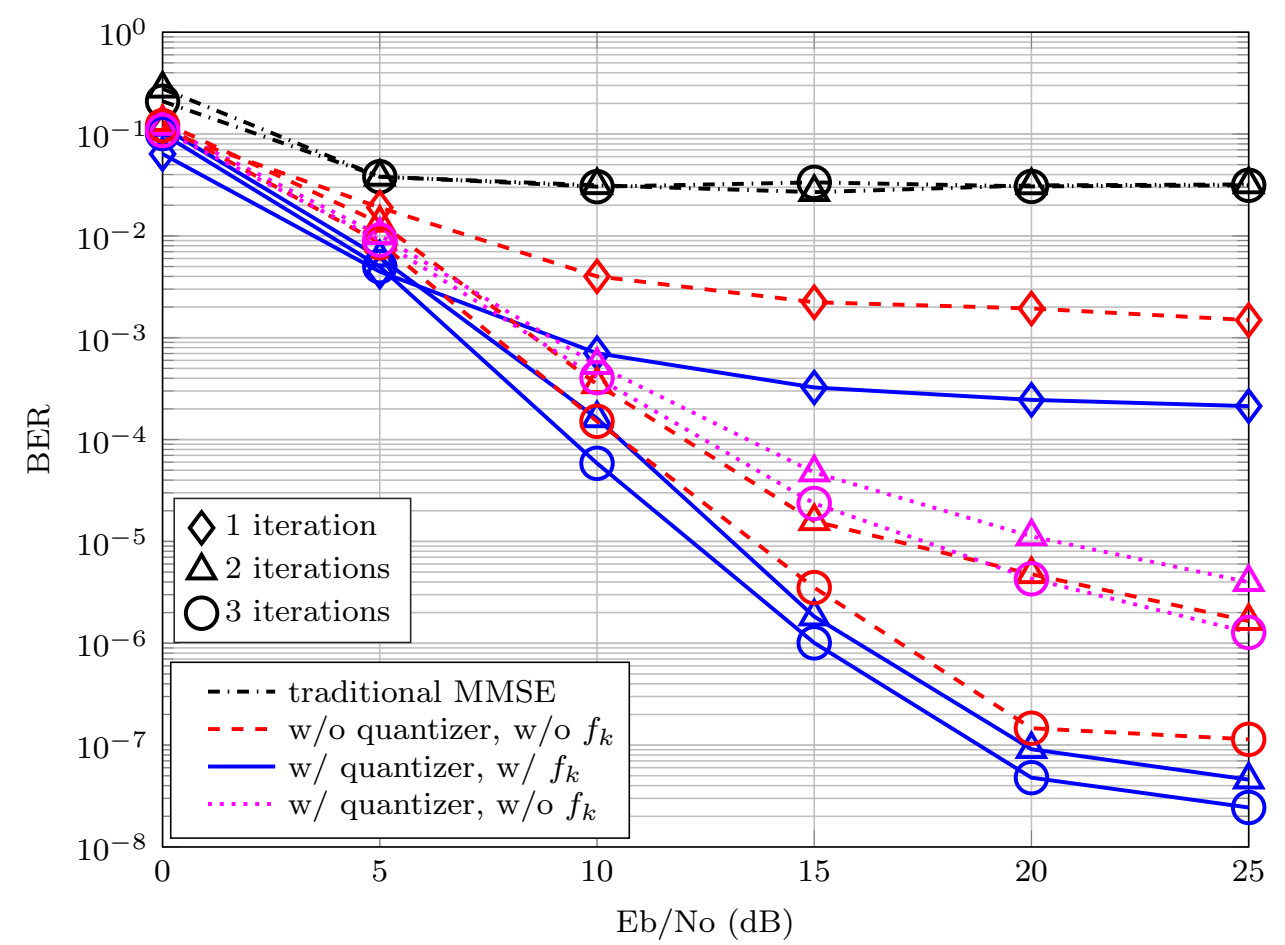

Figure 3.5: $N_{t}=12$ and $N_{r}=32$. BER performance of IDD schemes under perfect CSI.

\section{4}

\section{Summary}

This chapter has presented adaptive channel estimators and an IDD scheme for 1-bit large-scale MIMO systems. The proposed adaptive channel estimators have obtained good MSE performance with low computational cost. Moreover, an IDD scheme equipped with the LRA-LMMSE receive filter has been developed for 1-bit systems. The simulation results have shown a great performance gain after several iterations. Furthermore, an adaptive channel decoder has been devised using a quantizer together with scaling factors for further performance improvement. 


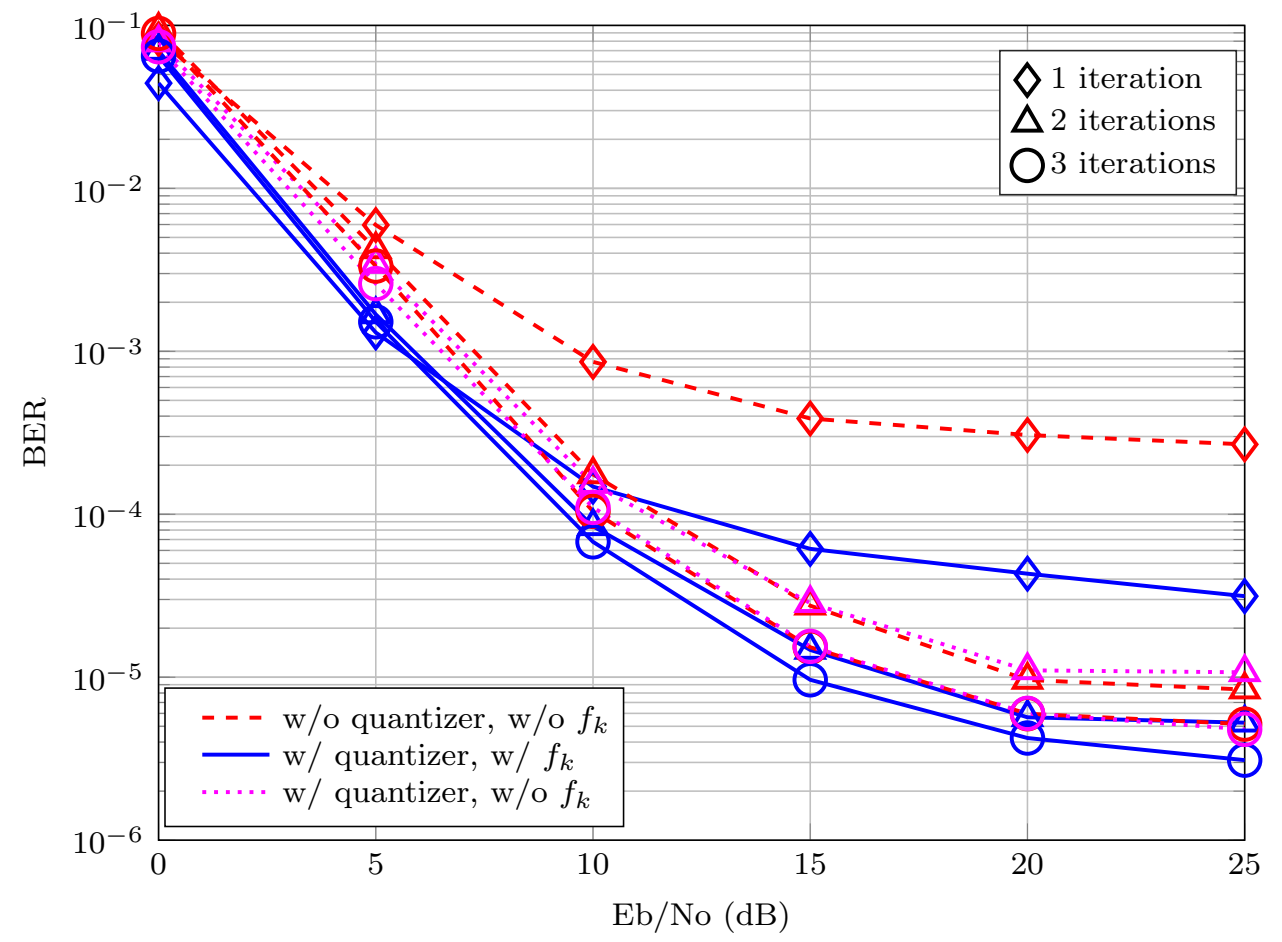

Figure 3.6: $N_{t}=9$ and $N_{r}=32$. BER performance of IDD schemes using BLMMSE channel estimation with $\tau=70$ pilot symbols. 


\section{4}

\section{Uniform-oversampling-rate based System Design}

The majority of current works about 1-bit quantized systems operate at the Nyquist-sampling rate, where only one sample is obtained in a Nyquist interval. To increase the information rate, oversampling (or faster-than-Nyquist signaling) is applied so that more samples are obtained in one Nyquist interval. The first work about 1-bit quantized signals with oversampling has been reported in [64], which shows a great advantage in terms of the achievable rate. For Gaussian noisy channels, the authors in [65] have demonstrated the advantage of oversampling in capacity. Furthermore, in [66] the authors have investigated the influence of different pulse shaping filters on the 1-bit quantized systems with oversampling. The results show that when using rootraised-cosine (RRC) filters, information rates can be increased.

More recently, several works have investigated 1-bit quantization with oversampling in MIMO systems. The study in [74] considers time-of-arrival estimation for systems with 1-bit quantization and oversampling and proposes corresponding performance bounds. The study in [71] has proposed carrier phase estimation and given lower bounds on complex channel parameter estimation for 1-bit oversampled systems based on [72]. In the study in [67] the BLMMSE channel estimator is applied to the MIMO channel with 1-bit quantization and oversampling using the simplifying assumption of uncorrelated noise samples which then yields performance degradation especially at low SNR and high oversampling factors. The authors in [68] have derived channel estimation algorithms for such systems. To reduce the additional computational cost caused by the extra samples resulting from oversampling, the sliding window technique is proposed while making the signal detection [69], where each transmission block is separated into several sub-blocks for further signal processing.

In this chapter, low-resolution aware (LRA) channel estimation and signal detection algorithms are developed for 1-bit oversampled large-scale MU-MIMO systems. Although the received signals are quantized to 1 bit, the computations after the 1-bit ADCs of all algorithms compared are performed at a higher resolution ( 8 bits or higher). Unlike prior work on 1-bit MIMO with oversampling [67], the main property of oversampled systems, which is the 
correlation of the filtered noise, is considered in this thesis. From the simulation results, the application of oversampling at the receiver can lead to significantly better performance.

Throughout this chapter, the system model is firstly illustrated and the correlation of filtered noise is then analyzed. Section 4.3 presents the proposed channel estimators, compares the complexity and gives the CRBs on MSE. Section 4.4 shows the sliding window based receivers for the 1-bit oversampled system.

\section{1}

\section{System Model}

The system model is depicted in Fig. 4.1, where $M$ is the oversampling factor. In the uplink, assuming perfect synchronization the received oversam-

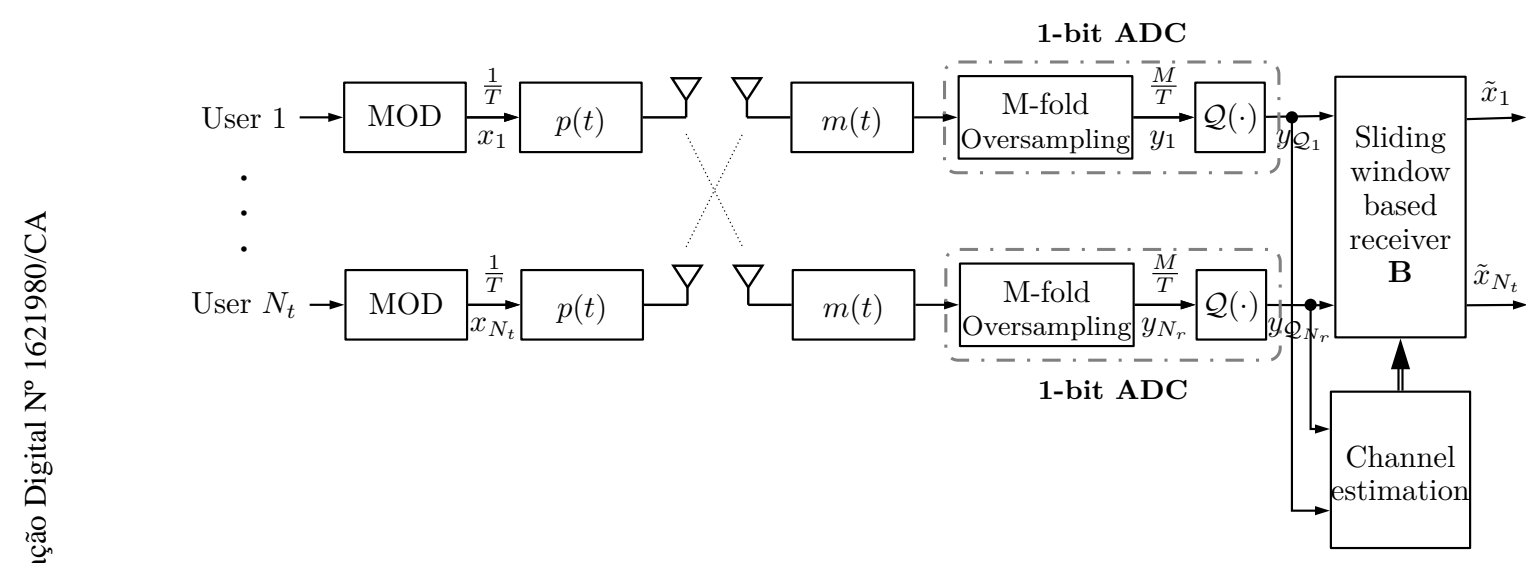

Figure 4.1: System model of 1-bit MIMO system with oversampling at the receiver

pled signal $\mathbf{y} \in \mathbb{C}^{M N_{r} N \times 1}$ can be expressed as

$$
\mathbf{y}=\mathbf{H x}+\mathbf{n},
$$

where $\mathbf{x} \in \mathbb{C}^{N N_{t} \times 1}$ contains IID transmitted symbols from $N_{t}$ terminals, each with block length $N$. The vector $\mathbf{x}$ is arranged as

$$
\mathbf{x}=\left[\begin{array}{llllll}
x_{1,1} & \cdots & x_{N, 1} & x_{1,2} & \cdots & x_{N, N_{t}}
\end{array}\right]^{T},
$$

where $x_{i, j}$ corresponds to the transmitted symbol of terminal $j$ at time instant $i$. Each symbol has unit power so that $E\left\{\left|x_{i, j}\right|^{2}\right\}=1$. The vector $\mathbf{n}$ represents the filtered oversampled noise expressed by

$$
\mathbf{n}=\left(\mathbf{I}_{N_{r}} \otimes \mathbf{G}\right) \mathbf{w}
$$

with $\mathbf{w} \sim \mathcal{C N}\left(\mathbf{0}_{3 M N_{r} N}, \sigma_{n}^{2} \mathbf{I}_{3 M N_{r} N}\right)$. Note that the noise samples are described such that each entry of $\mathbf{n}$ has the same statistical properties (such as the same variances). Since in digital domain the receive filter has a length of $2 M N+1$ 
samples, $3 M N$ unfiltered noise samples in the noise vector $\mathbf{w}$ need to be considered for the description of an interval of $M N$ samples of the filtered noise $\mathbf{n}$. The matrix $\mathbf{G} \in \mathbb{R}^{M N \times 3 M N}$ is a Toeplitz matrix that contains the impulse response of the receive filter $m(t)$ at different time instants and is shown as

$\mathbf{G}=\left[\begin{array}{ccccccc}m(-N T) & m\left(-N T+\frac{1}{M} T\right) & \ldots & m(N T) & 0 & \ldots & 0 \\ 0 & m(-N T) & \ldots & m\left(N T-\frac{1}{M} T\right) & m(N T) & \ldots & 0 \\ \vdots & \vdots & \ddots & \vdots & \vdots & \ddots & \vdots \\ 0 & 0 & \ldots & m(-N T) & m\left(-N T+\frac{1}{M} T\right) & \ldots & m(N T)\end{array}\right]$

where $T$ is the symbol period and $M$ denotes the oversampling rate. The equivalent channel matrix $\mathbf{H}$ is described as

$$
\mathbf{H}=\left[\mathbf{I}_{N_{r}} \otimes \mathbf{Z}\left(\mathbf{I}_{N} \otimes \mathbf{u}\right)\right]\left(\mathbf{H}^{\prime} \otimes \mathbf{I}_{N}\right),
$$

where $\mathbf{H}^{\prime} \in \mathbb{C}^{N_{r} \times N_{t}}$ is the channel matrix for non-oversampled systems and $\mathbf{u}$ is an oversampling vector with length $M$, which has the form

$$
\mathbf{u}=\left[\begin{array}{llll}
0 & \cdots & 0 & 1
\end{array}\right]^{T}
$$

The matrix $\mathbf{Z} \in \mathbb{R}^{M N \times M N}$ is a Toeplitz matrix that contains the coefficients of $z(t)$ at different time instants, where $z(t)$ is the convolution of the pulse shaping filter $p(t)$ and the matched filter $m(t)$ given by

$$
\mathbf{Z}=\left[\begin{array}{cccc}
z(0) & z\left(\frac{T}{M}\right) & \ldots & z\left(N T-\frac{1}{M} T\right) \\
z\left(-\frac{T}{M}\right) & z(0) & \ldots & z\left(N T-\frac{2}{M} T\right) \\
\vdots & \vdots & \ddots & \vdots \\
z\left(-N T+\frac{1}{M} T\right) & z\left(-N T+\frac{2}{M} T\right) & \ldots & z(0)
\end{array}\right] .
$$

In particular, $M=1$ refers to the non-oversampling case.

Let $\mathcal{Q}(\cdot)$ represent the 1-bit quantization function, the resulting quantized signal $\mathbf{y}_{\mathcal{Q}}$ is given by

$$
\mathbf{y}_{\mathcal{Q}}=\mathcal{Q}(\mathbf{y})=\mathcal{Q}\left(\mathbf{y}^{R}\right)+j \mathcal{Q}\left(\mathbf{y}^{I}\right)
$$

The real and imaginary parts of $\mathbf{y}$ are quantized element-wised to $\left\{ \pm \frac{1}{\sqrt{2}}\right\}$ based on the sign. The factor $\frac{1}{\sqrt{2}}$ is to make the power of each quantized signal to be one.

\section{2}

\section{Noise covariance matrix $C_{n}$}

With (4-3) the auto-correlation matrix of filtered noise $\mathbf{C}_{\mathbf{n}}$ is calculated as

$$
\mathbf{C}_{\mathbf{n}}=\sigma_{n}^{2}\left(\mathbf{I}_{N_{r}} \otimes \mathbf{G G}^{H}\right)
$$


For non-oversampled system $(M=1),(4-9)$ is reduced to

$$
\mathbf{C}_{\mathbf{n}}=\sigma_{n}^{2} \mathbf{I}_{N N_{r}}
$$

However, for oversampled system $(M \geq 2)$ (4-9) cannot be further simplified due to the correlation of oversampled samples. The off-diagonal elements will appear in the matrix $\mathbf{G G}^{H}$. One example is shown in the following figures, where $m(t)$ is assumed to be a normalized RRC filter with different roll-off factors, $M=2$ and $N=10$.
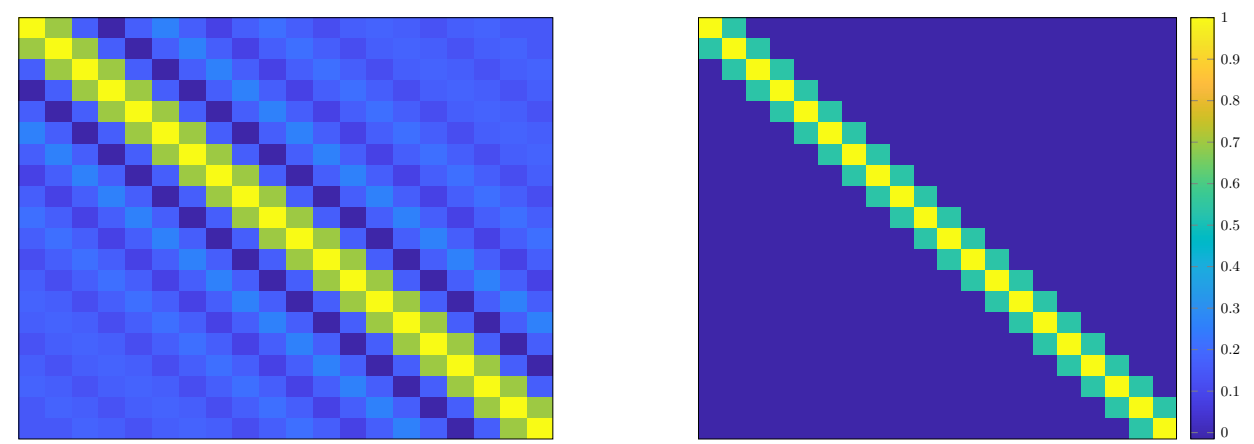

Figure 4.2: Matrix representation of $\mathbf{G G}^{H}$ Figure 4.3: Matrix representation of $\mathbf{G G}{ }^{H}$ with roll-off factor 0.1 with roll-off factor 0.9

We can see that the lower the roll-off factors the more off-diagonal elements appear in $\mathbf{G G}^{H}$, which means that for systems with low roll-off factors it is important to consider $\mathbf{C}_{\mathbf{n}}$ as a full matrix rather than a simplified diagonal matrix as assumed in [67].

\section{3}

\section{Pilot-based Channel Estimation}

Based on the Bussgang decomposition [32] the nonlinear system (4-8) can be reformulated into a statistically equivalent linear system. From this linear model, low-resolution aware LS, LMMSE and LMS channel estimation algorithms are developed for 1-bit oversampled systems and their computational costs are also evaluated. The fundamental estimation limit is also examined in this section by deriving a Bayesian framework and bounds on channel estimates for both non-oversampled and oversampled systems.

In a standard uplink implementation, the CSI is estimated at the BS and then used to detect the data symbols transmitted from the $N_{t}$ users. Each transmission block is divided into two sub-blocks: one for pilots and another for the data symbols. Pilots are either located at the beginning of each block or spread according to a desired pattern [70]. During the training phase, each terminal simultaneously transmits $\tau$ pilot symbols to the BS, which yields 


$$
\mathbf{y}_{p}=\mathbf{H} \mathbf{x}_{p}+\mathbf{n}_{p}
$$

Vectorizing and inserting (4-5) into (4-11) we get

$$
\begin{aligned}
\mathbf{y}_{p} & =\left(\mathbf{x}_{p}^{T} \otimes \mathbf{I}_{N_{r}}\right) \operatorname{vec}(\mathbf{H})+\mathbf{n}_{p} \\
& =\left[\mathbf{x}_{p}^{T} \otimes \mathbf{I}_{N_{r}} \otimes \mathbf{Z}\left(\mathbf{I}_{\tau} \otimes \mathbf{u}\right)\right] \operatorname{vec}\left(\mathbf{H}^{\prime} \otimes \mathbf{I}_{\tau}\right)+\mathbf{n}_{p} \\
& =\boldsymbol{\Phi}_{p} \mathbf{h}^{\prime}+\mathbf{n}_{p},
\end{aligned}
$$

where $\mathbf{h}^{\prime}=\operatorname{vec}\left(\mathbf{H}^{\prime}\right)$ and the equivalent pilot matrix is

$$
\boldsymbol{\Phi}_{p}=\left[\mathbf{x}_{p}^{T} \otimes \mathbf{I}_{N_{r}} \otimes \mathbf{Z}\left(\mathbf{I}_{\tau} \otimes \mathbf{u}\right)\right]\left[\mathbf{I}_{N_{t}} \otimes\left(\mathbf{e}_{1} \otimes \mathbf{I}_{N_{r}} \otimes \mathbf{e}_{1}+\cdots+\mathbf{e}_{\tau} \otimes \mathbf{I}_{N_{r}} \otimes \mathbf{e}_{\tau}\right)\right] .
$$

The vector $\mathbf{x}_{p} \in \mathbb{C}^{\tau N_{t} \times 1}$ contains the transmitted pilots and $\mathbf{e}_{n} \in \mathbb{R}^{\tau \times 1}$ represents a column vector with a one in the $n$th element and zeros elsewhere. After processing by 1-bit ADCs, the quantized signal can be expressed as

$$
\mathbf{y}_{\mathcal{Q}_{p}}=\mathcal{Q}\left(\boldsymbol{\Phi}_{p} \mathbf{h}^{\prime}+\mathbf{n}_{p}\right)=\tilde{\boldsymbol{\Phi}}_{p} \mathbf{h}^{\prime}+\tilde{\mathbf{n}}_{p}
$$

where $\tilde{\boldsymbol{\Phi}}_{p}=\mathbf{A}_{p} \boldsymbol{\Phi}_{p} \in \mathbb{C}^{M \tau N_{r} \times N_{t} N_{r}}$ and $\tilde{\mathbf{n}}_{p}=\mathbf{A}_{p} \mathbf{n}_{p}+\mathbf{n}_{q} \in \mathbb{C}^{M \tau N_{r} \times 1}$. The vector $\mathbf{n}_{q}$ is the statistically equivalent quantization noise with covariance matrix $\mathbf{C}_{\mathbf{n}_{q}}=\mathbf{C}_{\mathbf{y}_{\mathcal{Q}_{p}}}-\mathbf{A}_{p} \mathbf{C}_{\mathbf{y}_{p}} \mathbf{A}_{p}$. The matrix $\mathbf{A}_{p} \in \mathbb{R}^{M \tau N_{r} \times M \tau N_{r}}$ is the Bussgangbased linear operator chosen independently from $\mathbf{y}_{p}$ and is given by

$$
\mathbf{A}_{p}=\mathbf{C}_{\mathbf{y}_{p} \mathbf{y}_{\mathcal{Q}_{p}}}^{H} \mathbf{C}_{\mathbf{y}_{p}}^{-1}=\sqrt{\frac{2}{\pi}} \mathbf{K}, \quad \text { with } \quad \mathbf{K}=\operatorname{diag}\left(\mathbf{C}_{\mathbf{y}_{p}}\right)^{-\frac{1}{2}} .
$$

where $\mathbf{C}_{\mathbf{y}_{p} \mathbf{y}_{\mathcal{Q}_{p}}}$ denotes the cross-correlation matrix between the received signal $\mathbf{y}_{p}$ and its quantized signal $\mathbf{y}_{\mathcal{Q}_{p}}$

$$
\mathbf{C}_{\mathbf{y}_{p} \mathbf{y}_{\mathcal{Q}_{p}}}=\sqrt{\frac{2}{\pi}} \mathbf{K C}_{\mathbf{y}_{p}}
$$

The formulas of (4-15) and (4-16) involve the auto-correlation matrix $\mathbf{C}_{\mathbf{y}_{p}}$ :

$$
\mathbf{C}_{\mathbf{y}_{p}}=\boldsymbol{\Phi}_{p} \mathbf{R}_{\mathbf{h}^{\prime}} \boldsymbol{\Phi}_{p}^{H}+\mathbf{C}_{\mathbf{n}_{p}}
$$

where $\mathbf{R}_{\mathbf{h}^{\prime}}=E\left\{\mathbf{h}^{\prime} \mathbf{h}^{\prime H}\right\}$.

\subsection{1}

\section{Standard LS Channel Estimator}

The work in [54] has proposed the standard LS estimator for 1-bit non-oversampled systems. Similar to this, the extension of this estimator to oversampled systems is

$$
\begin{aligned}
\hat{\mathbf{h}}_{\text {Standard LS }}^{\prime} & =\underset{\overline{\mathbf{h}^{\prime}}}{\arg \min }\left\|\mathbf{y}_{\mathcal{Q}_{p}}-\boldsymbol{\Phi}_{p} \overline{\mathbf{h}}^{\prime}\right\|^{2} \\
& =\left(\boldsymbol{\Phi}_{p}^{H} \boldsymbol{\Phi}_{p}\right)^{-1} \boldsymbol{\Phi}_{p}^{H} \mathbf{y}_{\mathcal{Q}_{p}} .
\end{aligned}
$$


The advantage of this estimator is that no priori information is needed at the receiver. However, the issue with this estimator, when applied with 1bit quantization, is that the channel estimate $\hat{\mathbf{h}}^{\prime}$ scales with the amplitude associated with the quantizer, which then corresponds to a biased estimation.

\subsection{2}

\section{LRA-LS Channel Estimator}

Based on the linear equivalent system model in (4-14), the LRA-LS channel estimator is obtained by solving the following optimization problem:

$$
\begin{aligned}
\hat{\mathbf{h}}_{\text {LRA-LS }}^{\prime} & =\underset{\overline{\mathbf{h}}^{\prime}}{\arg \min }\left\|\mathbf{y}_{\mathcal{Q}_{p}}-\tilde{\boldsymbol{\Phi}}_{p} \overline{\mathbf{h}}^{\prime}\right\|^{2} \\
& =\left(\tilde{\boldsymbol{\Phi}}_{p}^{H} \tilde{\boldsymbol{\Phi}}_{p}\right)^{-1} \tilde{\boldsymbol{\Phi}}_{p}^{H} \mathbf{y}_{\mathcal{Q}_{p}} .
\end{aligned}
$$

Compared to the standard LS channel estimator, the proposed estimator has taken $\mathbf{R}_{\mathbf{h}^{\prime}}$ into consideration in order to obtain the linear operator $\mathbf{A}_{p}$.

\subsection{3}

\section{LRA-LMMSE Channel Estimator}

The LMMSE channel estimator has the advantage of superior MSE performance to that of the LS channel estimator. Based on the statistically equivalent linear model in (4-14), the oversampling based LRA-LMMSE channel estimator is proposed. The optimal filter is given by

$$
\begin{aligned}
\mathbf{W}_{\text {LRA-LMMSE }} & =\underset{\mathbf{W}}{\arg \min } E\left\{\left\|\mathbf{h}^{\prime}-\mathbf{W y}_{\mathcal{Q}_{p}}\right\|^{2}\right\} \\
& =\mathbf{R}_{\mathbf{h}^{\prime}} \tilde{\boldsymbol{\Phi}}^{H} \mathbf{C}_{\mathbf{y}_{\mathcal{Q}_{p}}}^{-1},
\end{aligned}
$$

where

$$
\mathbf{C}_{\mathbf{y}_{\mathcal{Q}_{p}}}=\frac{2}{\pi}\left(\sin ^{-1}\left(\mathbf{K C}_{\mathbf{y}_{p}}^{R} \mathbf{K}\right)+j \sin ^{-1}\left(\mathbf{K C}_{\mathbf{y}_{p}}^{I} \mathbf{K}\right)\right) .
$$

The resulting LRA-LMMSE channel estimator is then

$$
\hat{\mathbf{h}}_{\text {LRA-LMMSE }}^{\prime}=\mathbf{R}_{\mathbf{h}^{\prime}} \tilde{\boldsymbol{\Phi}}^{H} \mathbf{C}_{\mathbf{y}_{\mathcal{Q}_{p}}}^{-1} \mathbf{y}_{\mathcal{Q}_{p}}
$$

Proof. See Appendix B.

Note that when $M=1,(4-22)$ reduces to the same as that of the BLMMSE channel estimator in [10].

\subsection{4}

\section{LRA-LMS Channel Estimator}

Since for large-scale MIMO we have $N_{r} \gg N_{t}$, in order to reduce the computational complexity the multiplications and divisions involving large matrices, whose dimensions contain $N_{r}$ elements, need to be avoided. For this reason, the channel from $N_{t}$ users to only one receive antenna $n_{r}$ is concentrated 


$$
\mathbf{y}_{\mathcal{Q}_{p}}^{n_{r}}=\tilde{\mathbf{\Phi}}_{p}^{n_{r}} \mathbf{h}^{\prime n_{r}}+\tilde{\mathbf{n}}_{p}^{n_{r}},
$$

where $\mathbf{y}_{\mathcal{Q}_{p}}^{n_{r}}=\left[y_{\mathcal{Q}_{p}}^{n_{r}}(1), y_{\mathcal{Q}_{p}}^{n_{r}}(2), \ldots, y_{\mathcal{Q}_{p}}^{n_{r}}(M \tau)\right]^{T}$ and $\mathbf{h}^{\prime n_{r}} \in \mathbb{C}^{N_{t} \times 1}$ is the $n_{r}$ th row of $\mathbf{H}^{\prime}$. Different from $\tilde{\boldsymbol{\Phi}}_{p}$ in (4-14), $\tilde{\boldsymbol{\Phi}}_{p}^{n_{r}} \in \mathbb{C}^{M \tau \times N_{t}}$ is an equivalent pilot matrix to the $n_{r}$ th receive antenna. The sliding window technique is proposed together with the LMS channel estimation. Fig. 4.4 shows the basic mechanism, which combines the adjacent symbol-rate-sampled symbols together to estimate the instantaneous channel parameters, since in oversampled systems the interference from adjacent symbol-rate-sampled symbols should be considered. The first window contains the first $M l_{\text {win }}$ oversampled samples and the second contains the next $M l_{\text {win }}$ samples until the last window. Note that only one symbol-rate-sampled symbol (or $M$ oversampled samples) is shifted for the subsequent window.

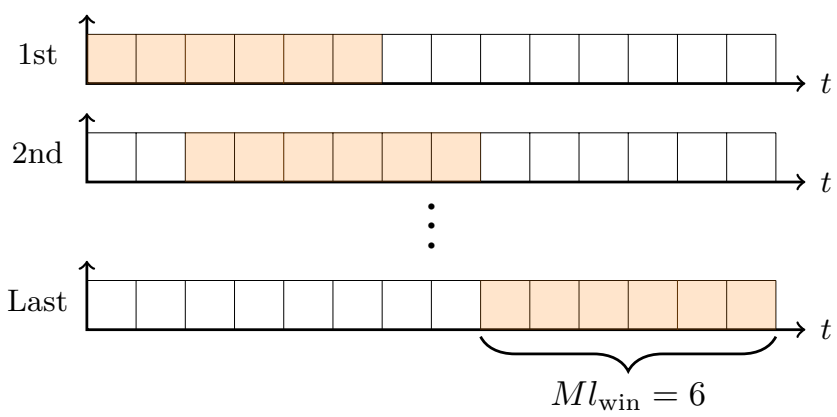

Figure 4.4: Illustration of the sliding window at each receive antenna when $l_{\text {win }}=3$ and $M=2$, where $l_{\text {win }}$ is the window length representing the number of non-oversampled symbols.

Based on (4-23), the received signal at the $n$th window can be expressed as

$$
\mathbf{y}_{\mathcal{Q}_{p}}^{n_{r}}(n)=\tilde{\mathbf{\Phi}}_{p}^{n_{r}}(n) \mathbf{h}^{\prime n_{r}}+\tilde{\mathbf{n}}_{p}^{n_{r}}(n),
$$

where $\mathbf{y}_{\mathcal{Q}_{p}}^{n_{r}}(n)=\left[y_{\mathcal{Q}_{p}}^{n_{r}}(M(n-1)+1), \ldots, y_{\mathcal{Q}_{p}}^{n_{r}}\left(M(n-1)+M l_{\text {win }}\right)\right]^{T}$ and $\tilde{\boldsymbol{\Phi}}_{p}^{n_{r}}(n)=\mathbf{A}_{p}^{n_{r}}(n) \boldsymbol{\Phi}_{p}^{n_{r}}(n) \in \mathbb{C}^{M l_{\text {win }} \times N_{t}}$ contains the transmit pilot sequences in the $n$th window.

The optimization problem that leads to the proposed LRA-LMS channel estimation algorithm can be stated as

$$
\hat{\mathbf{h}}_{\text {LRA-LMS }}^{\prime n_{r}}(n)=\underset{{\overline{\mathbf{h}^{\prime \prime}}}^{n_{r}}(n)}{\arg \min } \sum_{n=1}^{\tau-l_{\text {win }}+1}\left\|\mathbf{y}_{\mathcal{Q}_{p}}^{n_{r}}(n)-\tilde{\mathbf{\Phi}}_{p}^{n_{r}}(n){\overline{\mathbf{h}^{\prime}}}^{n_{r}}(n)\right\|^{2},
$$

where $\overline{\mathbf{h}}^{\prime n_{r}}(n)$ is the instantaneous estimate of $\mathbf{h}^{\prime n_{r}}$ in the $n$th window.

Taking the partial derivative of the objective function in (4-25) with respect to $\overline{\mathbf{h}}^{\prime n_{r}}(n)^{H}$, we obtain 


$$
\begin{aligned}
& \frac{\partial \sum_{n=1}^{\tau-l_{\text {win }}+1}\left\|\mathbf{y}_{\mathcal{Q}_{p}}^{n_{r}}(n)-\tilde{\boldsymbol{\Phi}}_{p}^{n_{r}}(n) \overline{\mathbf{h}}^{{ }^{n_{r}}}(n)\right\|^{2}}{\partial \overline{\mathbf{h}}^{n_{r}}(n)^{H}} \\
= & \sum_{n=1}^{\tau-l_{\text {win }}+1}-\tilde{\boldsymbol{\Phi}}_{p}^{n_{r}}(n)^{H}\left(\mathbf{y}_{\mathcal{Q}_{p}}^{n_{r}}(n)-\tilde{\boldsymbol{\Phi}}_{p}^{n_{r}}(n){\overline{\mathbf{h}^{\prime}}}^{n_{r}}(n)\right) \\
= & \sum_{n=1}^{\tau-l_{\text {win }}+1}-\tilde{\boldsymbol{\Phi}}_{p}^{n_{r}}(n)^{H} \mathbf{e}^{n_{r}}(n) .
\end{aligned}
$$

The recursion of the proposed LRA-LMS algorithm is

$$
{\overline{\mathbf{h}^{\prime}}}^{n_{r}}(n+1)={\overline{\mathbf{h}^{\prime}}}^{n_{r}}(n)+\mu \tilde{\boldsymbol{\Phi}}_{p}^{n_{r}}(n)^{H} \mathbf{e}^{n_{r}}(n), \quad n=1, \ldots, \tau-l_{\text {win }}+1,
$$

where the constant step size $\mu$ fulfills

$$
0<\mu<\frac{2}{\gamma_{\max }}
$$

$\gamma_{\max }$ is the largest eigenvalue of $\mathbf{C}_{\tilde{\boldsymbol{\Phi}}_{p}^{n_{r}}(n)}$, which is $E\left\{\tilde{\boldsymbol{\Phi}}_{p}^{n_{r}}(n) \tilde{\mathbf{\Phi}}_{p}^{n_{r}}(n)^{H}\right\}$.

Proof. See Appendix C.

The proposed adaptive channel estimator is summarized in Algorithm 2, where $\mathbf{x}_{p}(n) \in \mathbb{C}^{l_{\text {win }} N_{t} \times 1}$ contains the pilot symbols in the $n$th window. Both $\mathbf{e}_{n}^{\prime} \in \mathbb{R}^{l_{\text {win }} \times 1}$ and $\mathbf{e}_{n}^{\prime \prime} \in \mathbb{R}^{N_{r} \times 1}$ represent all-zero column vectors except that the $n$th elements are ones.

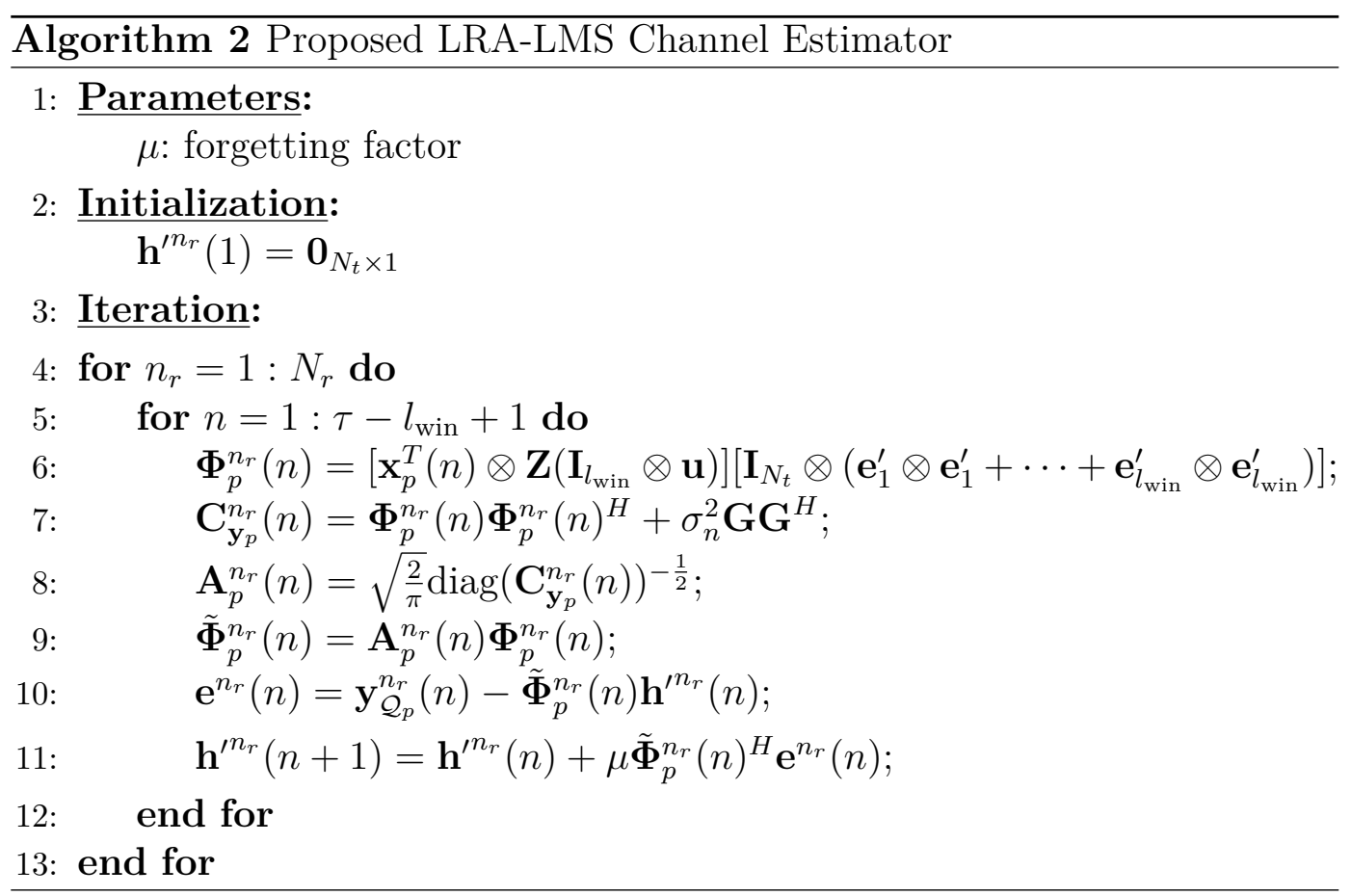

Fig. 4.5 shows the convergence performance of the proposed LRA-LMS channel estimator for each receive antenna. The proposed estimator achieves its steady state after $\tau=40$. 


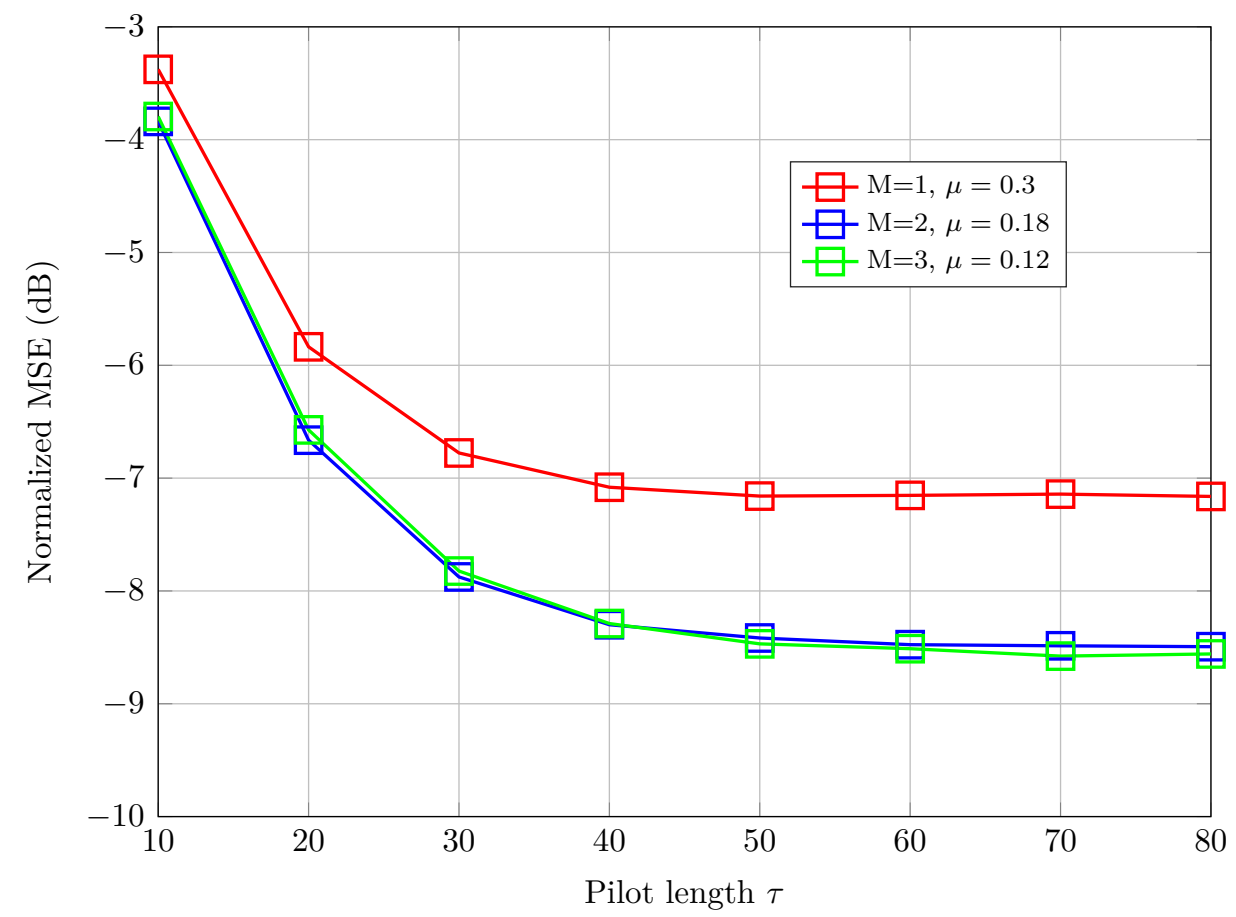

Figure 4.5: Convergence of the LRA-LMS channel estimator with $N_{t}=8$ and $N_{r}=64$ at $\mathrm{SNR}=20 \mathrm{~dB}$.

\subsection{5}

\section{Complexity Analysis}

The computational complexities of the proposed channel estimators are compared in this subsection. For the sake of simplification and a fair comparison among the estimators, $\mathbf{R}_{\mathbf{h}^{\prime}}$ is assumed to be an identity matrix since there is no linear operator used in the standard LS channel estimator. Table 4.1 shows the total required complex additions/subtractions and multiplications/divisions for obtaining the channel estimate $\hat{\mathbf{h}}^{\prime}$. More intuitively, Fig. 4.6 shows the total number of complex operations, which is a sum of complex additions and multiplications, as a function of the number of receive antennas $N_{r}$. Compared to other channel estimators, the LRA-LMS channel estimator consumes the lowest computational cost since there are no matrix inversions or large matrix multiplications in the algorithm. The comparisons in terms of MSE performance are shown in the subsection of numerical results.

\subsection{6}

\section{Estimation of $\mathbf{R}_{\mathrm{h}^{\prime}}$}

In practical environments, there is no prior information about $\mathbf{R}_{\mathbf{h}^{\prime}}$ at the receiver. In this subsection, an adaptive technique is proposed to recursively estimate $\mathbf{R}_{\mathbf{h}^{\prime}}$

$$
\hat{\mathbf{R}}_{\mathbf{h}^{\prime}}(n+1)=\lambda \hat{\mathbf{R}}_{\mathbf{h}^{\prime}}(n)+(1-\lambda) \hat{\mathbf{h}}^{\prime}(n) \hat{\mathbf{h}}^{\prime}(n)^{H}, \quad n=1, \ldots, \tau,
$$




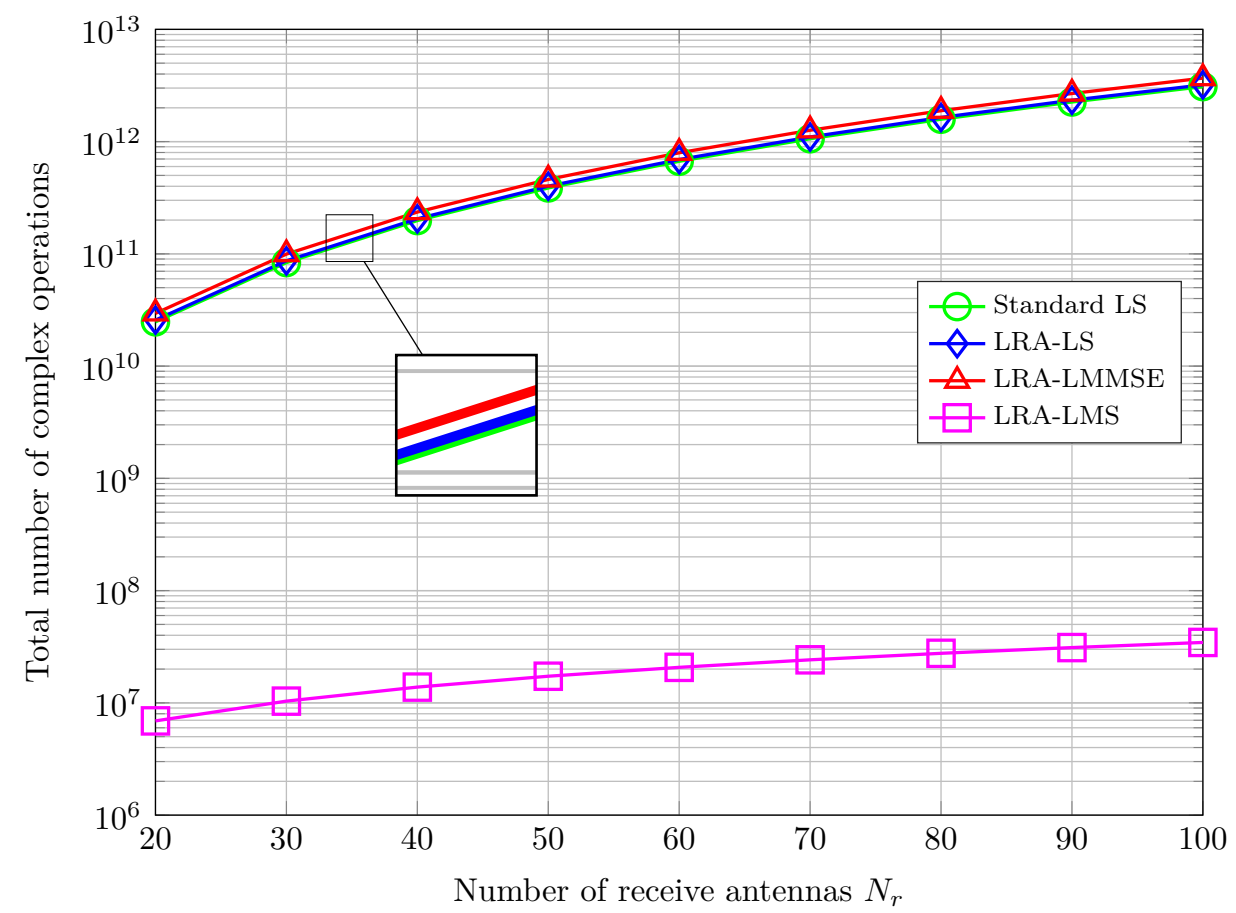

Figure 4.6: Computational complexity comparison among different channel estimators in an oversampled system $M=3$ with $\tau=20, l_{\text {win }}=3$ and $N_{t}=8$.

Table 4.1: Computational complexity of different channel estimators

\begin{tabular}{c||c|c}
\hline & Complex Additions/Subtractions & Complex Multiplications/Divisions \\
\hline \hline \multirow{3}{*}{ Standard LS } & $N_{r}^{3} N_{t}^{2}\left(N_{t}+M \tau^{3}+2 M \tau\right)$ & $N_{r}^{3} N_{t}^{2}\left(N_{t}+2 M \tau+\tau^{3} M\right)$ \\
& $-N_{r}^{2} N_{t}\left(M \tau+N_{t}\right)-2 N_{r} N_{t}$ & $+N_{r}^{2} \tau\left[\tau N_{t}^{2}+\left(\tau^{2}+1\right) M N_{t}+\tau^{2}+(1+M) \tau\right]$ \\
& $+M \tau^{2}(M \tau-1)$ & $+2 N_{r} N_{t}+M \tau^{2}(1+\tau)$ \\
\hline \multirow{3}{*}{ LRA-LS } & $N_{r}^{3} N_{t}\left[N_{t}^{2}+\left(M \tau^{3}+2 M \tau\right) N_{t}+2 M^{2} \tau^{2}\right]$ & $N_{r}^{3} N_{t}\left[N_{t}^{2}+\left(M \tau^{3}+2 M \tau\right) N_{t}+2 M^{2} \tau^{2}\right]$ \\
& $-N_{r}^{2} N_{t}\left(2 M \tau+N_{t}\right)-2 N_{r} N_{t}$ & $+N_{r}^{2} \tau\left[\tau N_{t}^{2}+\left(\tau^{2}+1\right) M N_{t}+\tau^{2}+\left(1+M+2 M^{2}\right) \tau\right]$ \\
& $+M \tau^{2}\left(M \tau-M-1+3 M^{2} \tau\right)$ & $+2 N_{r}\left(M \tau+N_{t}\right)+3 M^{3} \tau^{3}+M \tau^{2}(1+\tau)$ \\
\hline \multirow{3}{*}{ LRA-LMMSE } & $N_{r}^{3}\left[M \tau^{3} N_{t}^{2}+3 M^{2} \tau^{2} N_{t}+M^{3} \tau^{3}\right]$ & $N_{r}^{3}\left[M \tau^{3} N_{t}^{2}+3 M^{2} \tau^{2} N_{t}+M^{3} \tau^{3}\right]$ \\
& $-2 N_{r}^{2} M \tau N_{t}-N_{r}\left(M \tau+N_{t}\right)$ & $+N_{r}^{2} \tau\left[\tau N_{t}^{2}+\left(\tau^{2}+1\right) M N_{t}+\tau^{2}+\left(1+\tau+M+5 M^{2}\right) \tau\right]$ \\
& $+M \tau^{2}\left(M \tau-1+3 M^{2} \tau-M\right)$ & $+3 N_{r}+3 M^{3} \tau^{3}+M \tau^{2}(1+\tau)$ \\
\hline \multirow{2}{*}{ LRA-LMS } & $N_{r}\left(\tau-l_{\operatorname{win}}+1\right)\left[l_{\operatorname{win}}^{2} M\left(2 M N_{t}-M-1\right)\right.$ & $N_{r}\left(\tau-l_{\operatorname{win}}+1\right)\left[N_{t}+2 l_{\operatorname{win}} M\left(1+N_{t}\right)\right.$ \\
& $\left.+l_{\operatorname{win}}^{3} M\left(3 M^{2}+M+N_{t}^{2}\right)\right]$ & $+l_{\operatorname{win}}^{2}\left(1+N_{t}^{2}+2 M+2 M^{2} N_{t}+2 M^{2}\right)$ \\
& & $\left.+l_{\operatorname{win}}^{3}\left(N_{t}^{2} M+1+M N_{t}+M+3 M^{3}\right)\right]$ \\
\hline
\end{tabular}

where $\lambda$ is the forgetting factor $(0<\lambda<1)$ and $\hat{\mathbf{h}}^{\prime}(n)$ is the channel estimate at the Nyquist time instant n. Consider the system model

$$
\begin{aligned}
\mathbf{y}_{\mathcal{Q}}(n) & =\mathcal{Q}(\mathbf{H x}(n)+\mathbf{n}(n)) \\
& =\mathcal{Q}\left(\left(\mathbf{x}_{p}^{\prime T}(n) \otimes \mathbf{I}_{N_{r}} \otimes \mathbf{Z}^{\prime} \mathbf{u}\right) \mathbf{h}^{\prime}+\mathbf{n}(n)\right),
\end{aligned}
$$

where $\mathbf{y}_{\mathcal{Q}}(n)$ and $\mathbf{n}(n)$ are column vectors with size $M N_{r} \times 1$. Different from $\mathbf{x}_{p}(n)$ in Algorithm $2, \mathbf{x}^{\prime}{ }_{p}(n) \in \mathbb{C}^{N_{t} \times 1}$ contains pilot symbols from $N_{t}$ terminals at time instant $\mathrm{n}$. $\mathbf{Z}^{\prime} \in \mathbb{R}^{M \times M}$ is a simplified version of $\mathbf{Z}$ with $N=1$. The instantaneous estimate of $\mathbf{h}^{\prime}$ is calculated as

$$
\hat{\mathbf{h}}^{\prime}(n)=\left(\mathbf{x}_{p}^{\prime T}(n) \otimes \mathbf{I}_{N_{r}} \otimes \mathbf{Z}^{\prime} \mathbf{u}\right)^{+} \mathbf{y}_{\mathcal{Q}}(n)
$$


where the initial guess of $\hat{\mathbf{R}}_{\mathbf{h}^{\prime}}(1)$ is an identity matrix, as channel parameters are assumed to be uncorrelated and each has unit power.

\subsection{7}

\section{Cramér-Rao Bounds}

In this subsection, analytical bounds on the MSE are analyzed. Unlike the works in $[71,72]$, which have proposed the CRBs for the unbiased estimators, the existing CRBs are extended for the biased estimators. Two different types of CRBs are proposed depending on whether the prior information $\mathbf{R}_{\mathbf{h}^{\prime}}$ is known at the receiver, namely Bayesian CRB with known $\mathbf{R}_{\mathbf{h}^{\prime}}$ and general CRB with estimated $\mathbf{R}_{\mathbf{h}^{\prime}}$.

\subsubsection{1}

\section{Bayesian Cramér-Rao Bounds}

The Bayesian bounds on the fundamental limits of estimation are derived for non-oversampled and oversampled systems. Without loss of generality, (412 ) is extended for considering the whole system and not just the pilots. The complex-valued model can be rewritten in the following real-valued form

$$
\left[\begin{array}{c}
\mathbf{y}^{R} \\
\mathbf{y}^{I}
\end{array}\right]=\left[\begin{array}{cc}
\boldsymbol{\Phi}^{R} & -\boldsymbol{\Phi}^{I} \\
\boldsymbol{\Phi}^{I} & \boldsymbol{\Phi}^{R}
\end{array}\right]\left[\begin{array}{c}
\mathbf{h}^{\prime R} \\
\mathbf{h}^{\prime I}
\end{array}\right]+\left[\begin{array}{c}
\mathbf{n}^{R} \\
\mathbf{n}^{I}
\end{array}\right]
$$

Let $\tilde{\mathbf{h}}^{\prime}=\left[\mathbf{h}^{\prime R} ; \mathbf{h}^{\prime I}\right]$ be the unknown parameter vector, since the real and imaginary parts are independent, the Bayesian information matrix (BIM) [73] for the quantized signal is defined as

$$
\mathbf{J}_{\mathbf{y}_{\mathcal{Q}}}\left(\tilde{\mathbf{h}}^{\prime}\right)=\mathbf{J}_{\mathbf{y}_{\mathcal{Q}}^{R}}\left(\tilde{\mathbf{h}}^{\prime}\right)+\mathbf{J}_{\mathbf{y}_{\mathcal{Q}}^{I}}\left(\tilde{\mathbf{h}}^{\prime}\right)
$$

where

$$
\left[\mathbf{J}_{\mathbf{y}_{\mathcal{Q}}^{R / I}}\left(\tilde{\mathbf{h}^{\prime}}\right)\right]_{i j} \triangleq E_{\mathbf{y}_{\mathcal{Q}}^{R / I}, \tilde{\mathbf{h}^{\prime}}}\left\{\frac{\partial \ln p\left(\mathbf{y}_{\mathcal{Q}}^{R / I}, \tilde{\mathbf{h}}^{\prime}\right)}{\partial\left[\tilde{\mathbf{h}}^{\prime}\right]_{i}} \frac{\partial \ln p\left(\mathbf{y}_{\mathcal{Q}}^{R / I}, \tilde{\mathbf{h}^{\prime}}\right)}{\partial\left[\tilde{\mathbf{h}}^{\prime}\right]_{j}}\right\}
$$

with $\left[\tilde{\mathbf{h}}^{\prime}\right]_{i}$ and $\left[\tilde{\mathbf{h}}^{\prime}\right]_{j}$ being the elements of $\tilde{\mathbf{h}}^{\prime}$. The expression in (4-34) can be divided into two parts:

$$
\left[\mathbf{J}_{\mathbf{y}_{\mathcal{Q}}^{R / I}}\left(\tilde{\mathbf{h}}^{\prime}\right)\right]_{i j}=\left[\mathbf{J}_{\mathbf{y}_{\mathcal{Q}}^{R / I}}^{D}\left(\tilde{\mathbf{h}}^{\prime}\right)\right]_{i j}+\left[\mathbf{J}_{\mathbf{y}_{\mathcal{Q}}^{R / I}}^{P}\left(\tilde{\mathbf{h}}^{\prime}\right)\right]_{i j},
$$

where we define

$$
\begin{aligned}
{\left[\mathbf{J}_{\mathbf{y}_{\mathcal{Q}}^{R / I}}^{D}\left(\tilde{\mathbf{h}}^{\prime}\right)\right]_{i j} \triangleq E_{\mathbf{y}_{\mathcal{Q}}^{R / I} \mid \tilde{\mathbf{h}}^{\prime}}\left\{\frac{\partial \ln p\left(\mathbf{y}_{\mathcal{Q}}^{R / I} \mid \tilde{\mathbf{h}}^{\prime}\right)}{\partial\left[\tilde{\mathbf{h}}^{\prime}\right]_{i}} \frac{\partial \ln p\left(\mathbf{y}_{\mathcal{Q}}^{R / I} \mid \tilde{\mathbf{h}}^{\prime}\right)}{\partial\left[\tilde{\mathbf{h}}^{\prime}\right]_{j}}\right\} } \\
{\left[\mathbf{J}_{\mathbf{y}_{\mathcal{Q}}^{R / I}}^{P}\left(\tilde{\mathbf{h}}^{\prime}\right)\right]_{i j} \triangleq E_{\tilde{\mathbf{h}}^{\prime}}\left\{\frac{\partial \ln p\left(\tilde{\mathbf{h}}^{\prime}\right)}{\partial\left[\tilde{\mathbf{h}}^{\prime}\right]_{i}} \frac{\partial \ln p\left(\tilde{\mathbf{h}^{\prime}}\right)}{\partial\left[\tilde{\mathbf{h}}^{\prime}\right]_{j}}\right\} . }
\end{aligned}
$$


To transform the real-valued $\mathbf{J}_{\mathbf{y}_{\mathcal{Q}}}\left(\tilde{\mathbf{h}}^{\prime}\right)$ back to the complex domain $\mathbf{J}_{\mathbf{y}_{\mathcal{Q}}}\left(\mathbf{h}^{\prime}\right), \mathbf{J}_{\mathbf{y}_{\mathcal{Q}}}\left(\tilde{\mathbf{h}}^{\prime}\right)$ is defined with the following structure:

$$
\mathbf{J}_{\mathbf{y}_{\mathcal{Q}}}\left(\tilde{\mathbf{h}}^{\prime}\right)=\left[\begin{array}{cc}
\mathbf{J}_{\mathbf{y}_{\mathcal{Q}}}^{R R}\left(\tilde{\mathbf{h}}^{\prime}\right) & \mathbf{J}_{\mathbf{y}_{\mathcal{Q}}}^{R I}\left(\tilde{\mathbf{h}}^{\prime}\right) \\
\mathbf{J}_{\mathbf{y}_{\mathcal{Q}}}^{I R}\left(\tilde{\mathbf{h}}^{\prime}\right) & \mathbf{J}_{\mathbf{y}_{\mathcal{Q}}}^{I I}\left(\tilde{\mathbf{h}}^{\prime}\right)
\end{array}\right]
$$

and apply the chain rule to get:

$$
\mathbf{J}_{\mathbf{y}_{\mathcal{Q}}}\left(\mathbf{h}^{\prime}\right)=\frac{1}{4}\left(\mathbf{J}_{\mathbf{y}_{\mathcal{Q}}}^{R R}\left(\tilde{\mathbf{h}}^{\prime}\right)+\mathbf{J}_{\mathbf{y}_{\mathcal{Q}}}^{I I}\left(\tilde{\mathbf{h}}^{\prime}\right)\right)+\frac{j}{4}\left(\mathbf{J}_{\mathbf{y}_{\mathcal{Q}}}^{R I}\left(\tilde{\mathbf{h}}^{\prime}\right)-\mathbf{J}_{\mathbf{y}_{\mathcal{Q}}}^{I R}\left(\tilde{\mathbf{h}}^{\prime}\right)\right)
$$

where $\mathbf{J}_{\mathbf{y}_{\mathcal{Q}}}^{R R}\left(\tilde{\mathbf{h}}^{\prime}\right), \mathbf{J}_{\mathbf{y}_{\mathcal{Q}}}^{R I}\left(\tilde{\mathbf{h}}^{\prime}\right), \mathbf{J}_{\mathbf{y}_{\mathcal{Q}}}^{I R}\left(\tilde{\mathbf{h}}^{\prime}\right)$ and $\mathbf{J}_{\mathbf{y}_{\mathcal{Q}}}^{I I}\left(\tilde{\mathbf{h}}^{\prime}\right)$ have the same dimensions $N_{r} N_{t} \times$ $N_{r} N_{t}$. The variance of the estimator $\hat{\mathbf{h}}^{\prime}\left(\mathbf{y}_{\mathcal{Q}}\right)$ is lower bounded by

$$
\operatorname{Var}\left\{{\hat{h^{\prime}}}_{i}\left(\mathbf{y}_{\mathcal{Q}}\right)\right\} \geq\left[\mathbf{J}_{\mathbf{y}_{\mathcal{Q}}}^{-1}\left(\mathbf{h}^{\prime}\right)\right]_{i i}
$$

\section{BIM for systems sampling at symbol rate}

For non-oversampled systems, i.e, $M=1$, the covariance matrix of the equivalent noise vector $\mathbf{n}$ is $\mathbf{C}_{\mathbf{n}}=\sigma_{n}^{2} \mathbf{I}_{N N_{r}}$. With the independence of the real and imaginary parts, the log-likelihood function can be expressed as

$$
\ln p\left(\mathbf{y}_{\mathcal{Q}} \mid \tilde{\mathbf{h}}^{\prime}\right)=\sum_{k=1}^{N N_{r}}\left[\ln p\left(\left[\mathbf{y}_{\mathcal{Q}}^{R}\right]_{k} \mid \tilde{\mathbf{h}}^{\prime}\right)+\ln p\left(\left[\mathbf{y}_{\mathcal{Q}}^{I}\right]_{k} \mid \tilde{\mathbf{h}}^{\prime}\right)\right]
$$

with

$$
\begin{aligned}
& p\left(\left[\mathbf{y}_{\mathcal{Q}}^{R}\right]_{k}= \pm \frac{1}{\sqrt{2}} \mid \tilde{\mathbf{h}}^{\prime}\right)=Q\left(\mp \frac{\left[\boldsymbol{\Phi}^{R} \mathbf{h}^{\prime R}-\boldsymbol{\Phi}^{I} \mathbf{h}^{\prime I}\right]_{k}}{\sigma_{n} / \sqrt{2}}\right) \\
& p\left(\left[\mathbf{y}_{\mathcal{Q}}^{I}\right]_{k}= \pm \frac{1}{\sqrt{2}} \mid \tilde{\mathbf{h}}^{\prime}\right)=Q\left(\mp \frac{\left[\boldsymbol{\Phi}^{I} \mathbf{h}^{\prime}{ }^{R}+\boldsymbol{\Phi}^{R} \mathbf{h}^{\prime I}\right]_{k}}{\sigma_{n} / \sqrt{2}}\right)
\end{aligned}
$$

where $Q(x)=\frac{1}{\sqrt{2 \pi}} \int_{x}^{\infty} \exp \left(-\frac{u^{2}}{2}\right) d u$. Inserting (4-41) into (4-36), we obtain

$$
\begin{aligned}
{\left[\mathbf{J}_{\mathbf{y}_{\mathcal{Q}}}^{D}\left(\tilde{\mathbf{h}}^{\prime}\right)\right]_{i j} } & =-E\left\{\frac{\partial^{2} \ln p\left(\mathbf{y}_{\mathcal{Q}} \mid \tilde{\mathbf{h}}^{\prime}\right)}{\partial\left[\tilde{\mathbf{h}}^{\prime}\right]_{i} \partial\left[\tilde{\mathbf{h}}^{\prime}\right]_{j}}\right\} \\
& =\left[\mathbf{J}_{\mathbf{y}_{\mathcal{Q}}^{R}}^{D}\left(\tilde{\mathbf{h}}^{\prime}\right)\right]_{i j}+\left[\mathbf{J}_{\mathbf{y}_{\mathcal{Q}}^{I}}^{D}\left(\tilde{\mathbf{h}}^{\prime}\right)\right]_{i j}
\end{aligned}
$$

With the derivative of the $Q(x)$ function, the real part in $(4-36)\left[\mathbf{J}_{\mathbf{y}_{\mathcal{Q}}^{R}}^{D}\left(\tilde{\mathbf{h}^{\prime}}\right)\right]_{i j}$ is given by

$$
\begin{aligned}
& {\left[\mathbf{J}_{\mathbf{y}_{\mathcal{Q}}^{R}}^{D}\left(\tilde{\mathbf{h}^{\prime}}\right)\right]_{i j}=\sum_{k=1}^{N N_{r}}-E\left\{\frac{\partial^{2} \ln p\left(\left[\mathbf{y}_{\mathcal{Q}}^{R}\right]_{k} \mid \tilde{\mathbf{h}^{\prime}}\right)}{\partial\left[\tilde{\mathbf{h}^{\prime}}\right]_{i} \partial\left[\tilde{\mathbf{h}}^{\prime}\right]_{j}}\right\}=\frac{1}{\pi \sigma_{n}^{2}}} \\
& \times \sum_{k=1}^{N N_{r}} \frac{\exp \left(-\frac{\left[\boldsymbol{\Phi}^{R} \mathbf{h}^{\prime R}-\boldsymbol{\Phi}^{I} \mathbf{h}^{\prime I}\right]_{k}^{2}}{\sigma_{n}^{2} / 2}\right) \frac{\partial\left[\boldsymbol{\Phi}^{R} \mathbf{h}^{\prime}{ }^{R}-\boldsymbol{\Phi}^{I} \mathbf{h}^{\prime I}\right]_{k}}{\partial\left[\tilde{\mathbf{h}}^{\prime}\right]_{i}} \frac{\partial\left[\boldsymbol{\Phi}^{R} \mathbf{h}^{\prime R}-\boldsymbol{\Phi}^{I} \mathbf{h}^{\prime I}\right]_{k}}{\partial\left[\tilde{\mathbf{h}}^{\prime}\right]_{j}}}{Q\left(\frac{\left[\Phi^{R} \mathbf{h}^{\prime R}-\Phi^{I} \mathbf{h}^{I I}\right]_{k}}{\sigma_{n} / \sqrt{2}}\right) Q\left(-\frac{\left[\boldsymbol{\Phi}^{R} \mathbf{h}^{\prime R}-\boldsymbol{\Phi}^{I} \mathbf{h}^{\prime}\right]_{k}}{\sigma_{n} / \sqrt{2}}\right)} .
\end{aligned}
$$

The derivation for the imaginary part $\left[\mathbf{J}_{\mathbf{y}_{\mathcal{Q}}^{I}}^{D}\left(\tilde{\mathbf{h}}^{\prime}\right)\right]_{i j}$ is analogous.

By assuming that $\tilde{\mathbf{h}}^{\prime}$ is Gaussian distributed with zero mean and covari- 
ance matrix $\mathbf{C}_{\tilde{\mathbf{h}}^{\prime}}=\frac{1}{2} \mathbf{I}_{2} \otimes \mathbf{C}_{\mathbf{h}^{\prime}}, \ln p\left(\tilde{\mathbf{h}}^{\prime}\right)$ yields

$$
\ln p\left(\tilde{\mathbf{h}^{\prime}}\right)=-\frac{1}{2} N_{r} N_{t} \ln \left[(2 \pi)^{2 N_{r} N_{t}} \operatorname{det}\left(\mathbf{C}_{\tilde{\mathbf{h}}^{\prime}}\right)\right]-\frac{1}{2}{\tilde{\mathbf{h}^{\prime}}}^{T} \mathbf{C}_{\tilde{\mathbf{h}}^{\prime}}^{-1} \tilde{\mathbf{h}}^{\prime}
$$

Substituting (4-46) into (4-37), we obtain

$$
\mathbf{J}_{\mathbf{y}_{\mathcal{Q}}}^{P}\left(\tilde{\mathbf{h}}^{\prime}\right)=2 \mathbf{J}_{\mathbf{y}_{\mathcal{Q}}^{R / I}}^{P}\left(\tilde{\mathbf{h}}^{\prime}\right)=2 \mathbf{C}_{\tilde{\mathbf{h}}^{\prime}}^{-1}
$$

Finally, the resulting BIM is the summation of (4-44) and (4-47) as described by

$$
\mathbf{J}_{\mathbf{y}_{\mathcal{Q}}}\left(\tilde{\mathbf{h}}^{\prime}\right)=\mathbf{J}_{\mathbf{y}_{\mathcal{Q}}}^{D}\left(\tilde{\mathbf{h}}^{\prime}\right)+\mathbf{J}_{\mathbf{y}_{\mathcal{Q}}}^{P}\left(\tilde{\mathbf{h}}^{\prime}\right)
$$

\section{BIM for systems sampling at uniform oversampling rate}

When $M \geq 2$ the equivalent noise vector $\mathbf{n}$ consists of colored Gaussian noise samples. Computing $p\left(\mathbf{y}_{\mathcal{Q}}^{R / I} \mid \tilde{\mathbf{h}}^{\prime}\right)$ requires the orthant probabilities, which are not available or too difficult to compute. The authors in $[72,74]$ have introduced a lower bounding technique on the Fisher information for real-valued system. To employ this lower bounding technique in the complexvalued system, the work of [71] has come out. The lower bound of $\mathbf{J}_{\mathbf{y}_{\mathcal{Q}}^{R / I}}^{D}\left(\tilde{\mathbf{h}^{\prime}}\right)$ is calculated based on the first and second order moments as

$$
\mathbf{J}_{\mathbf{y}_{\mathcal{Q}}^{R / I}}^{D}\left(\tilde{\mathbf{h}}^{\prime}\right) \geq\left(\frac{\partial \mu_{\mathbf{y}_{\mathcal{Q}}^{R / I}}}{\partial \tilde{\mathbf{h}}^{\prime}}\right)^{T} \mathbf{C}_{\mathbf{y}_{\mathcal{Q}}^{R / I}}^{-1}\left(\frac{\partial \mu_{\mathbf{y}_{\mathcal{Q}}^{R / I}}}{\partial \tilde{\mathbf{h}}^{\prime}}\right)=\tilde{\mathbf{J}}_{\mathbf{y}_{Q}^{R / I}}^{D}\left(\tilde{\mathbf{h}}^{\prime}\right) .
$$

Since the lower-bounding technique is identical for the real and the imaginary parts, only the derivation of $\tilde{\mathbf{J}}_{\mathbf{y}_{\mathcal{Q}}^{R}}^{D}\left(\tilde{\mathbf{h}}^{\prime}\right)$ is presented. The mean value of the $k$ th received symbol is

$$
\begin{aligned}
{\left[\mu_{\mathbf{y}_{\mathcal{Q}}^{R}}\right]_{k} } & =\frac{1}{\sqrt{2}} \operatorname{Pr}\left(\left[\mathbf{y}_{\mathcal{Q}}\right]_{k}=+1 \mid \tilde{\mathbf{h}}^{\prime}\right)-\frac{1}{\sqrt{2}} \operatorname{Pr}\left(\left[\mathbf{y}_{\mathcal{Q}}\right]_{k}=-1 \mid \tilde{\mathbf{h}}^{\prime}\right) \\
& =\frac{1}{\sqrt{2}}\left[1-2 Q\left(\frac{\left[\boldsymbol{\Phi}^{R} \mathbf{h}^{\prime R}-\boldsymbol{\Phi}^{I} \mathbf{h}^{\prime}\right]_{k}}{\sqrt{\left[\mathbf{C}_{\mathbf{n}}\right]_{k k} / 2}}\right)\right]
\end{aligned}
$$

The partial derivative of (4-50) with respect to $\left[\tilde{\mathbf{h}^{\prime}}\right]_{i}$ is

$$
\frac{\partial\left[\mu_{\mathbf{y}_{\mathcal{Q}}^{R}}\right]_{k}}{\partial\left[\tilde{\mathbf{h}^{\prime}}\right]_{i}}=\frac{2 \exp \left(-\frac{\left[\boldsymbol{\Phi}^{R} \mathbf{h}^{\prime R}-\boldsymbol{\Phi}^{I} \mathbf{h}^{\prime I}\right]_{k}^{2}}{\left[\mathbf{C}_{n}\right]_{k k}}\right) \frac{\partial\left[\boldsymbol{\Phi}^{R} \mathbf{h}^{\prime R}-\boldsymbol{\Phi}^{I} \mathbf{h}^{\prime I}\right]_{k}}{\partial\left[\tilde{\mathbf{h}}^{\prime}\right]_{i}}}{\sqrt{2 \pi\left[\mathbf{C}_{\mathbf{n}}\right]_{k k}}} .
$$

The diagonal elements of the covariance matrix are given by

$$
\left[\mathbf{C}_{\mathbf{y}_{\mathcal{Q}}^{R}}\right]_{k k}=\frac{1}{2}-\left[\mu_{\mathbf{y}_{\mathcal{Q}}^{R}}\right]_{k}^{2}
$$

while the off-diagonal elements are calculated as

$$
\left[\mathbf{C}_{\mathbf{y}_{\mathcal{Q}}^{R}}\right]_{k n}=\operatorname{Pr}\left(z_{k}>0, z_{n}>0\right)+\operatorname{Pr}\left(z_{k} \leq 0, z_{n} \leq 0\right)-\frac{1}{2}-\left[\mu_{\mathbf{y}_{\mathcal{Q}}^{R}}\right]_{k}\left[\mu_{\mathbf{y}_{\mathcal{Q}}^{R}}\right]_{n}
$$


where $\left[z_{k}, z_{n}\right]^{T}$ is a bi-variate Gaussian random vector

$$
\left[\begin{array}{c}
z_{k} \\
z_{n}
\end{array}\right] \sim \mathcal{N}\left(\left[\begin{array}{l}
{\left[\boldsymbol{\Phi}^{R} \mathbf{h}^{\prime R}-\boldsymbol{\Phi}^{I} \mathbf{h}^{\prime I}\right]_{k}} \\
{\left[\boldsymbol{\Phi}^{R} \mathbf{h}^{\prime R}-\boldsymbol{\Phi}^{I} \mathbf{h}^{\prime I}\right]_{n}}
\end{array}\right], \frac{1}{2}\left[\begin{array}{ll}
{\left[\mathbf{C}_{\mathbf{n}}\right]_{k k}} & {\left[\mathbf{C}_{\mathbf{n}}\right]_{k n}} \\
{\left[\mathbf{C}_{\mathbf{n}}\right]_{n k}} & {\left[\mathbf{C}_{\mathbf{n}}\right]_{n n}}
\end{array}\right]\right)
$$

The lower bound for the imaginary part is derived in the same way. With the calculations above we get the lower bound of the BIM as

$$
\mathbf{J}_{\mathbf{y}_{\mathcal{Q}}}\left(\tilde{\mathbf{h}}^{\prime}\right) \geq \tilde{\mathbf{J}}_{\mathbf{y}_{\mathcal{Q}}}^{D}\left(\tilde{\mathbf{h}}^{\prime}\right)+\mathbf{J}_{\mathbf{y}_{\mathcal{Q}}}^{P}\left(\tilde{\mathbf{h}}^{\prime}\right)
$$

where the equality holds for $M=1$, as shown in [72] for the real valued CRB and in [71] for the complex valued CRB. Based on (4-40), the inverse of this BIM lower bound will result in an upper bound of the actual Bayesian CRB for oversampled systems.

\subsubsection{2}

\section{General Cramér-Rao Bounds}

When $\mathbf{R}_{\mathbf{h}^{\prime}}$ is unknown and needs to be estimated at the receiver, the Bayesian CRBs will not be applicable. We derive here general CRBs for the proposed channel estimators with estimated $\mathbf{R}_{\mathbf{h}^{\prime}}$.

Lemma 1 The proposed LRA channel estimators with combination of estimated $\hat{\mathbf{R}}_{\mathbf{h}^{\prime}}$ are biased channel estimators.

Proof. See Appendix D.

Since the proposed LRA channel estimators are biased, while calculating the CRBs, they should apply as

$$
\begin{aligned}
& \operatorname{Cov}\left\{\hat{\mathbf{h}}_{\text {bias }}^{\prime R}\right\} \geq \frac{\partial E\left\{\hat{\mathbf{h}}_{\text {bias }}^{R}\right\}}{\partial \mathbf{h}^{\prime R}}\left(\mathbf{J}_{\mathbf{y}_{\mathcal{Q}}}^{D^{R R}}\left(\mathbf{h}^{\prime R}\right)^{-1} \frac{\partial E\left\{\hat{\mathbf{h}}_{\text {bias }}^{R}\right\}}{\partial \mathbf{h}^{\prime R}}\right)^{T} \\
& \operatorname{Cov}\left\{\hat{\mathbf{h}}_{\text {bias }}^{I}\right\} \geq \frac{\partial E\left\{\hat{\mathbf{h}}_{\text {bias }}^{\prime I}\right\}}{\partial \mathbf{h}^{\prime I}}\left(\mathbf{J}_{\mathbf{y}_{\mathcal{Q}}}^{D^{I I}}\left(\mathbf{h}^{\prime I}\right)^{-1} \frac{\partial E\left\{\hat{\mathbf{h}}_{\text {bias }}^{I}\right\}}{\partial \mathbf{h}^{\prime I}}\right)^{T},
\end{aligned}
$$

where $\mathbf{J}_{\mathbf{y}_{\mathcal{Q}}}^{D^{R R}}\left(\mathbf{h}^{\prime R}\right)$ and $\mathbf{J}_{\mathbf{y}_{\mathcal{Q}}}^{D^{I I}}\left(\mathbf{h}^{\prime I}\right)$ are defined by

$$
\begin{aligned}
& {\left[\mathbf{J}_{\mathbf{y}_{\mathcal{Q}}}^{D^{R R}}\left(\mathbf{h}^{\prime R}\right)\right]_{i j} \triangleq E\left\{\frac{\partial \ln p\left(\mathbf{y}_{\mathcal{Q}} \mid \mathbf{h}^{\prime R}\right)}{\partial\left[\mathbf{h}^{\prime}\right]_{i}} \frac{\partial \ln p\left(\mathbf{y}_{\mathcal{Q}} \mid \mathbf{h}^{\prime R}\right)}{\partial\left[\mathbf{h}^{\prime R}\right]_{j}}\right\}} \\
& {\left[\mathbf{J}_{\mathbf{y}_{\mathcal{Q}}}^{D^{I I}}\left(\mathbf{h}^{\prime I}\right)\right]_{i j} \triangleq E\left\{\frac{\partial \ln p\left(\mathbf{y}_{\mathcal{Q}} \mid \mathbf{h}^{\prime I}\right)}{\partial\left[\mathbf{h}^{\prime I}\right]_{i}} \frac{\partial \ln p\left(\mathbf{y}_{\mathcal{Q}} \mid \mathbf{h}^{\prime I}\right)}{\partial\left[\mathbf{h}^{\prime I}\right]_{j}}\right\}}
\end{aligned}
$$

which are the upper left and lower right part of the $\mathbf{J}_{\mathbf{y}_{\mathcal{Q}}}^{D}\left(\tilde{\mathbf{h}}^{\prime}\right)$ (similar as (4-38)), respectively. 


\subsection{8}

\section{Numerical Results}

The simulation results presented here consider an uplink single-cell 1-bit large-scale MIMO system with $N_{t}=8$ and $N_{r}=64$. The modulation scheme is QPSK. The $m(t)$ and $p(t)$ filters are normalized RRC filters with a roll-off factor of 0.8 . The channel is assumed to experience block fading and the pilots are column-wise orthogonal with length 20. The SNR is defined as $10 \log \left(\frac{N_{t}}{\sigma_{n}^{2}}\right)$. The normalized MSE and SER performance plots are obtained by taking the average of 300 channel matrices, noise and symbol vectors.

For the LRA-LMS channel estimator, the window length $l_{\text {win }}$ is chosen as three to ensure low computational complexity. The step size $\mu$ is optimized according to the oversampling factor and SNR. In the simulation, $\mu$ varies between 0.05 and 0.3 . While recovering the transmitted symbols from the received quantized signal, the sliding-window based LMMSE detector [69] with window length equal to three $\left(l_{\text {win }}=3\right)$ and the estimate of the channel obtained by the proposed algorithms is applied in the system for obtaining both high accuracy and low computational cost.

The performance of the channel estimators is evaluated based on the channel model simulated in [75]. The channel for user $n_{t}$ is assumed Rayleigh distributed

$$
\mathbf{h}_{n_{t}}^{\prime}=\mathbf{R}_{r, n_{t}}^{\frac{1}{2}} \mathbf{h}_{w, n_{t}}^{\prime},
$$

where $\mathbf{R}_{r, n_{t}}$ denotes the receive correlation matrix with the following form

$$
\mathbf{R}_{r, n_{t}}=\left[\begin{array}{cccc}
1 & \rho_{n_{t}} & \ldots & \rho_{n_{t}}^{\left(N_{r}-1\right)} \\
\rho_{n_{t}}^{*} & 1 & \ldots & \rho_{n_{t}}^{\left(N_{r}-2\right)} \\
\vdots & \vdots & \ddots & \vdots \\
\rho_{n_{t}}^{*\left(N_{r}-1\right)} & \rho_{n_{t}}^{*\left(N_{r}-2\right)} & \ldots & 1
\end{array}\right]
$$

$\rho_{n_{t}}$ is the correlation index of neighboring antennas. $\left(\left|\rho_{n_{t}}\right|=0\right.$ represents an uncorrelated scenario and $\left|\rho_{n_{t}}\right|=1$ implies a fully correlated scenario). The elements of $\mathbf{h}_{w, n_{t}}^{\prime}$ are IID complex Gaussian random variables with zero mean and unit variance. All users are assumed to experience the same value of $\left|\rho_{n_{t}}\right|=|\rho|$ but different phases uniformly distributed over $2 \pi$. The overall channel model is summarized as

$$
\mathbf{H}^{\prime}=\left[\mathbf{h}_{1}^{\prime}, \mathbf{h}_{2}^{\prime}, \cdots, \mathbf{h}_{n_{t}}^{\prime}\right]
$$

and $\mathbf{R}_{\mathbf{h}^{\prime}}$ is calculated as 


$$
\mathbf{R}_{\mathbf{h}^{\prime}}=\left[\begin{array}{cccc}
\mathbf{R}_{r, 1} & \mathbf{0} & \ldots & \mathbf{0} \\
\mathbf{0} & \mathbf{R}_{r, 2} & \ldots & \mathbf{0} \\
\vdots & \vdots & \ddots & \vdots \\
\mathbf{0} & \mathbf{0} & \ldots & \mathbf{R}_{r, n_{t}}
\end{array}\right]
$$

\subsubsection{1}

\section{$R_{h^{\prime}}$ is known at the receiver}

In this subsection, the performance of the proposed LRA channel estimators with known $\mathbf{R}_{\mathbf{h}^{\prime}}$ at the receiver is evaluated. Fig. 4.7 and Fig. 4.8 compare the normalized MSE of the various channel estimators as a function of SNR in uncorrelated $(|\rho|=0)$ and correlated channel $(|\rho|=0.75)$, respectively. There is a $2 \mathrm{~dB}$ performance gain of the oversampled systems as compared to the non-oversampled systems for the LRA-LMMSE channel estimator at low SNR, whereas a much larger gain at high SNR. In both channels the LRALMMSE achieves the best MSE performance at the cost of high computational cost.

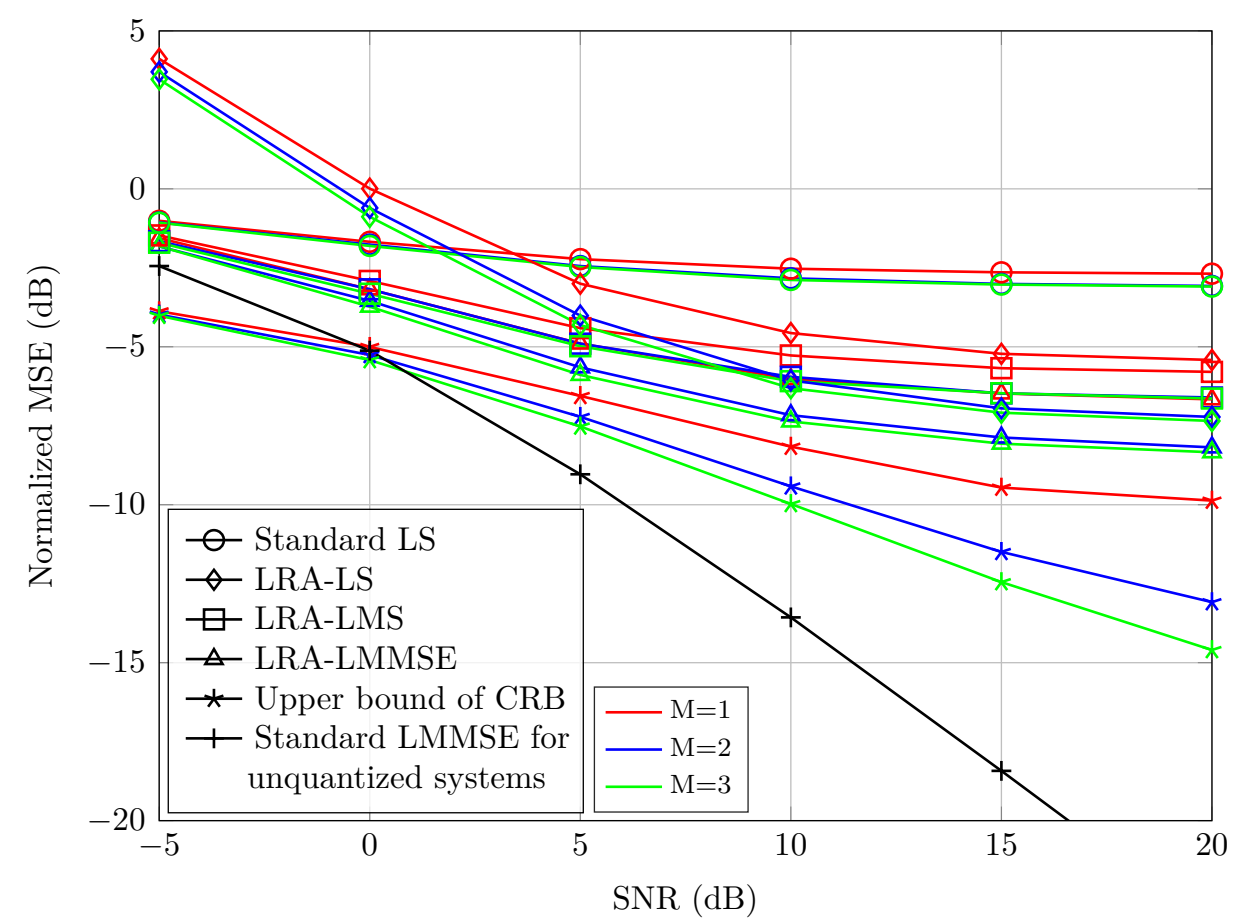

Figure 4.7: Normalized MSE comparisons of different channel estimators with known $\mathbf{R}_{\mathbf{h}^{\prime}}$ and $|\rho|=0$

In contrast, the LRA-LMS estimates the channel matrix $\mathbf{H}^{\prime}$ row by row. This approach can largely reduce the computational cost (shown in Fig. 4.6).Note that this separation into several rows may overlook the correlation of receive antennas. More specifically, the proposed LRA-LMS treats $\mathbf{R}_{r, n_{t}}$ as an identity matrix. As an amendment, the resulting estimated channel matrix 


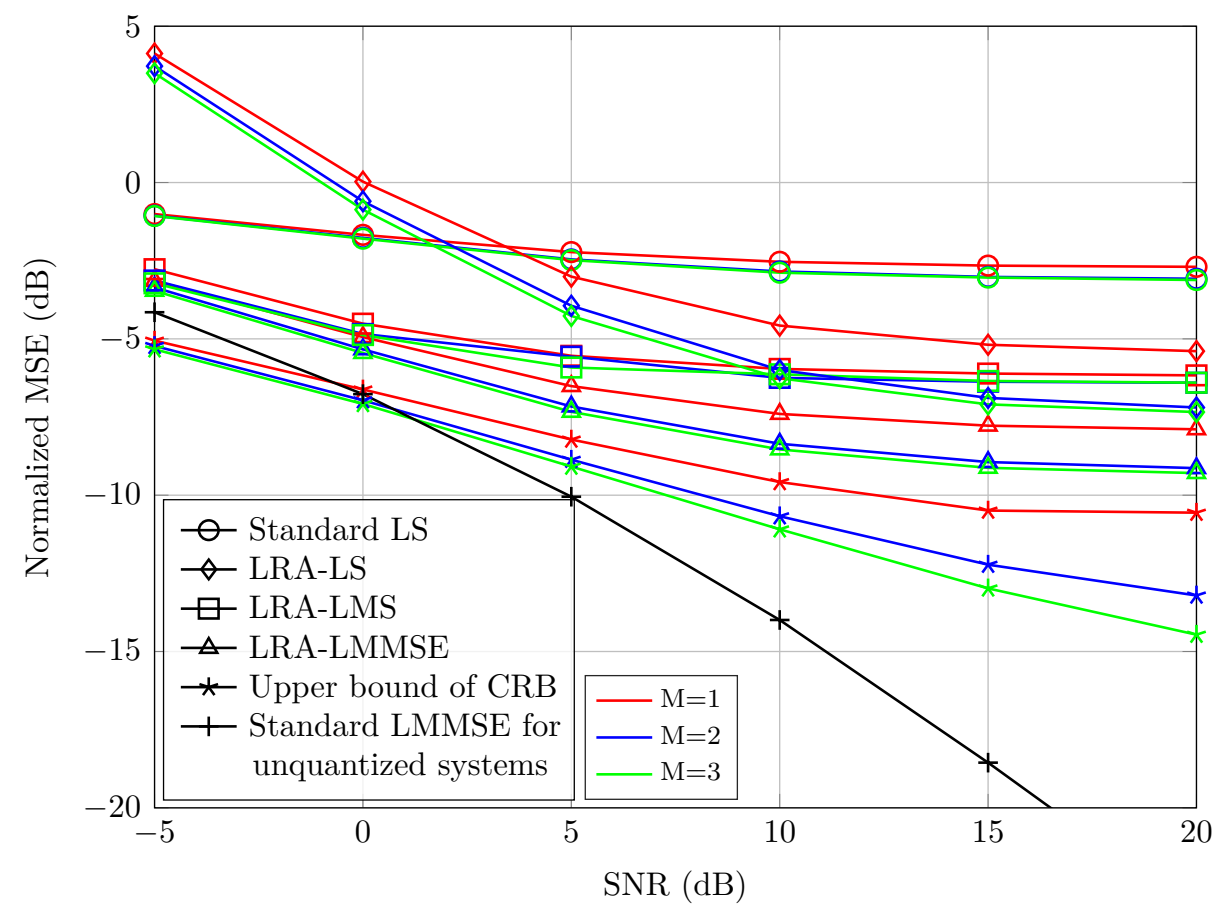

Figure 4.8: Normalized MSE comparisons of different channel estimators with known $\mathbf{R}_{\mathbf{h}^{\prime}}$ and $|\rho|=0.75$

$\hat{\mathbf{h}}_{\text {LRA-LMS }}^{\prime}$ needs to be multiplied with the square root of the receive correlation matrix $\mathbf{R}_{r_{n_{t}}}^{\frac{1}{2}}$, which can be derived from $\mathbf{R}_{\mathbf{h}^{\prime}}$ in (4-62). From the results, it can be seen that in both channels the LRA-LMS approaches the performance of the LRA-LMMSE at low SNR $(\leq 5 \mathrm{~dB})$, whereas at high SNR this performance gap becomes large.

The Bayesian CRBs illustrated in subsection 4.3.7.1 are also depicted in Fig. 4.7 and Fig. 4.8. Note that for the oversampled systems $(M \geq 2)$ the upper bounds of Bayesian CRBs are higher than the actual Bayesian CRBs, since they are derived from the lower bounds of Bayesian information. The black lines represent the standard LMMSE performance for the systems with unquantized signals, which can be treated as lower bounds for the systems with 1-bit quantized signals.

The LMMSE detector with sliding-window based SER performance of the system with the LRA-LMMSE estimated and perfect channel matrix are illustrated in Fig. 4.9, where the oversampled systems obviously outperform the non-oversampled systems. As described in section 4.2, Fig. 4.10 shows the MSE comparisons between LRA-LMMSE and simplified LMMSE [67] channel estimator in the system with $\tau=10$ and roll-off factor 0.1 . In this thesis, the correlation of filtered noise is taken into account, and hence $\mathbf{C}_{\mathbf{n}_{p}}$ is not a diagonal matrix in oversampled systems. It can be seen that at low SNR $(\leq 10 \mathrm{~dB})$ the performance of simplified LMMSE [67] is worse than the 


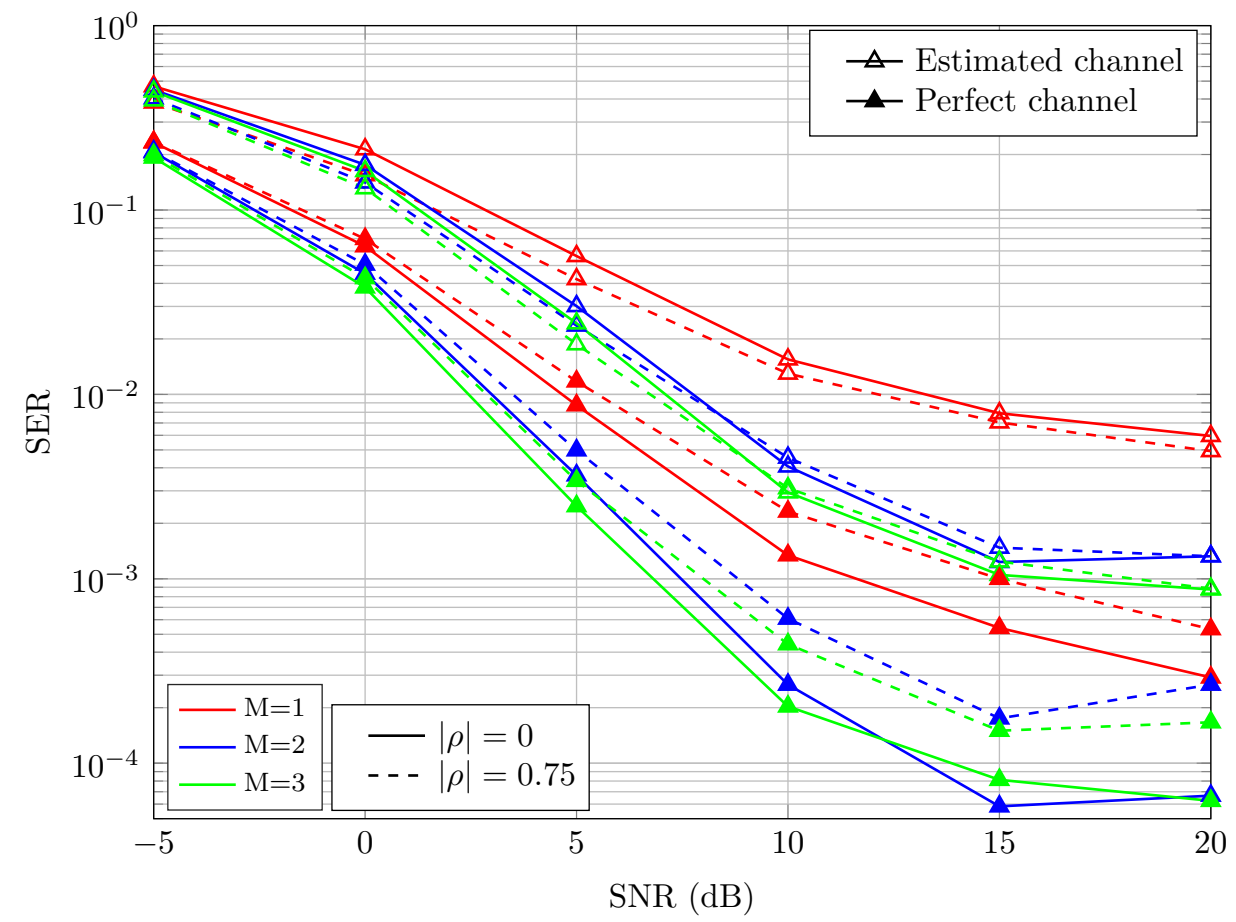

Figure 4.9: SER comparisons of different oversampling factors for the LRA-LMMSE channel estimator with known $\mathbf{R}_{\mathbf{h}^{\prime}}$.

proposed LRA-LMMSE, although they converge together at high SNR $(>10$ $\mathrm{dB})$. Another observation is that at low SNR the simplified LMMSE estimator with $M=3$ performs worse than that with $M=2$, which shows that the assumption in [67] is inaccurate.

\subsubsection{2}

\section{$R_{h^{\prime}}$ is unknown at the receiver}

Practically, $\mathbf{R}_{\mathbf{h}^{\prime}}$ is not known at the receiver. Fig. 4.11 shows the MSE performance of the LRA channel estimators by using the proposed adaptive recursion to estimate $\mathbf{R}_{\mathbf{h}^{\prime}}$, where $\lambda$ is set to 0.99 . It can be seen that the performance remains almost the same as Fig. 4.7, which shows that the proposed estimation of $\mathbf{R}_{\mathbf{h}^{\prime}}$ works well under uncorrelated channel.

While analyzing the general CRBs proposed in (4-55) and (4-56), instead of directly calculating the gradient of the expected value with respect to the channel vector $\frac{\partial E\left\{\hat{\mathbf{h}}^{\prime}{ }_{\text {bias }}\right\}}{\partial \mathbf{h}^{\prime / I}}$, this gradient is numerically evaluated, since there is an adaptive estimation technique inside the channel estimator, which makes the calculation more difficult. As one example, Fig. 4.12 shows the normalized MSE performance of the LRA-LS channel estimator with estimated $\hat{\mathbf{R}}_{\mathbf{h}^{\prime}}$ in (4-29) for estimating the first $N_{r}$ elements ${ }^{1}$ of $\mathbf{h}^{\prime 2}$ and its corresponding

${ }^{1}$ For the sake of simplicity, only first $N_{r}$ elements are considered, since for the large-scale MIMO there are $N_{t} N_{r}$ elements in $\mathbf{h}^{\prime R}$, which will cost much time for calculating the general CRBs. 


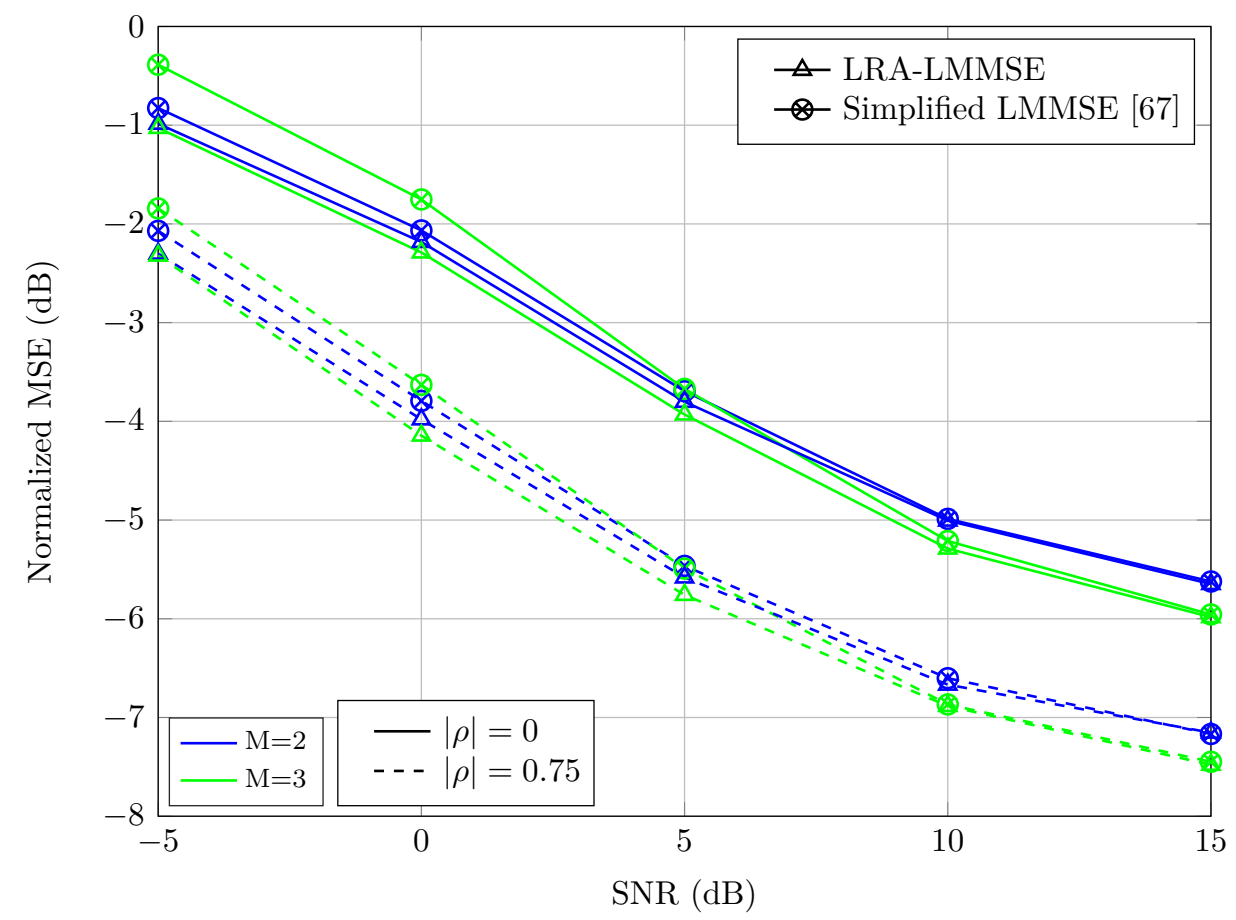

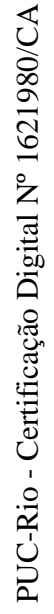

Figure 4.10: Normalized MSE comparisons between LRA-LMMSE and simplified LMMSE [67].

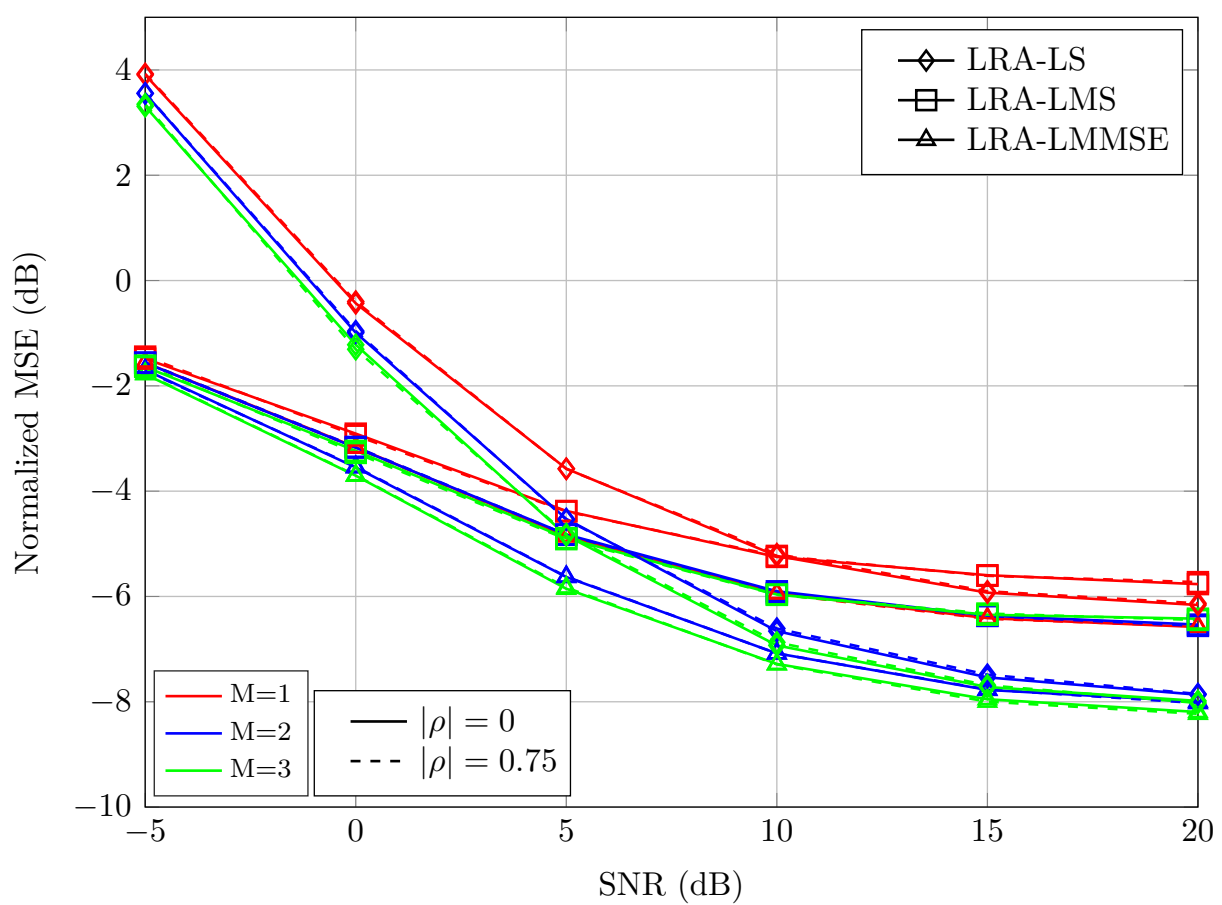

Figure 4.11: Normalized MSE comparisons of different channel estimators with adaptively estimated $\hat{\mathbf{R}}_{\mathbf{h}^{\prime}}$. 
numerically calculated general CRBs under uncorrelated channels $(|\rho|=0)$. More specifically, each element of the gradient vector $\frac{\partial E\left\{\hat{\mathbf{h}}^{\prime} \text { ias }\right\}}{\partial \mathbf{h}^{R / I}}$ is calculated with the following steps:

- increasing a small value $\delta$ (e.g. 0.1) in the corresponding element of $\mathbf{h}^{\prime R / I}$ - estimating the channel $\hat{\mathbf{h}}_{\text {bias }}^{R / I}$ with different transmit symbols and noises (e.g. 1000 different realizations)

- calculating the mean value of all estimates $E\left\{\hat{\mathbf{h}}_{\text {bias }}^{R / I}\right\}$, which will be divided by $\delta$.

These steps are repeated until all the elements in $\frac{\partial E\left\{\hat{\mathbf{h}}_{\text {bias }}^{R / I}\right\}}{\partial \mathbf{h}^{R / I}}$ are obtained.

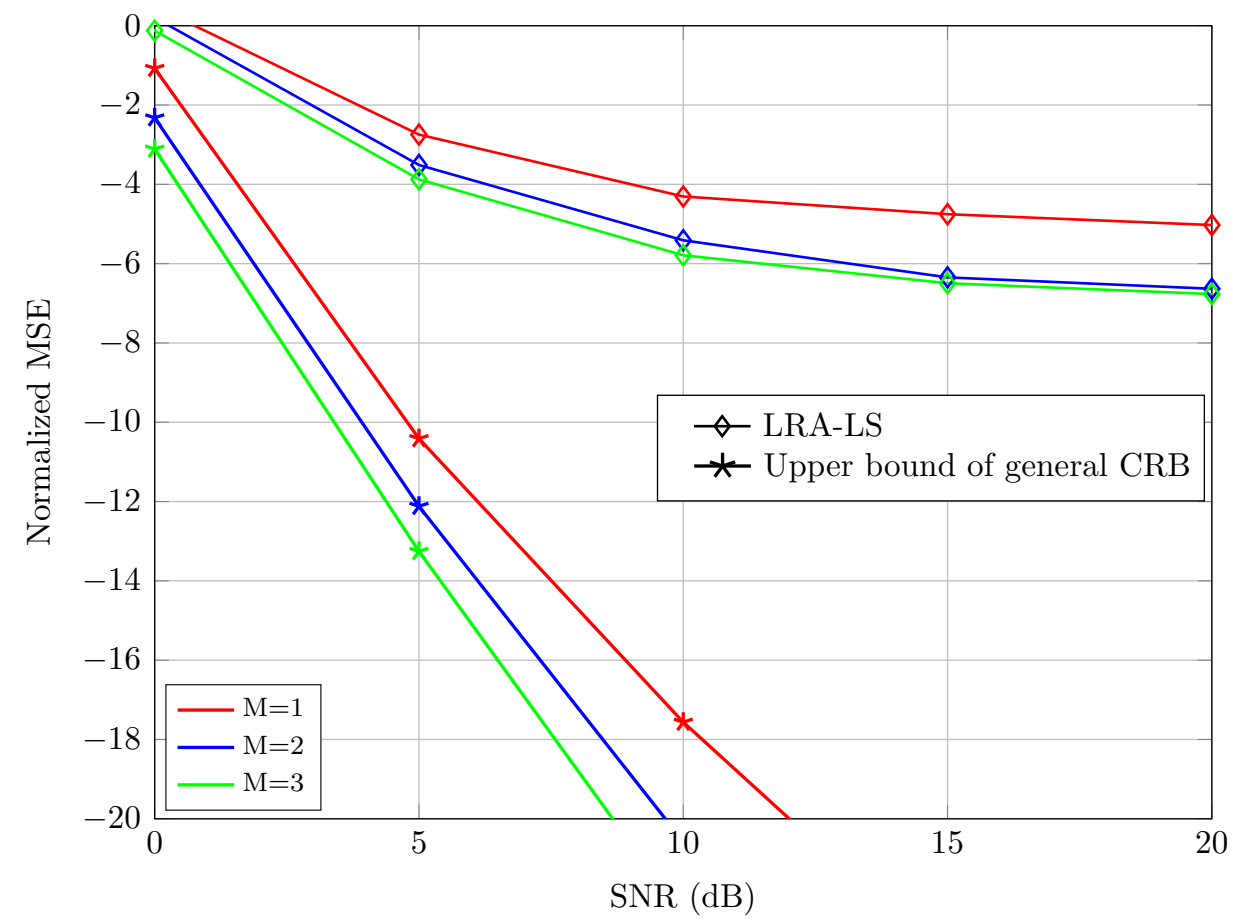

Figure 4.12: Normalized MSE comparisons of different oversampling factors for the LRA-LS channel estimator with estimated $\hat{\mathbf{R}}_{\mathbf{h}^{\prime}}$.

\subsubsection{3}

\section{1-bit or b-bit systems}

Fig. 4.13 shows the normalized MSE comparisons between 1-bit oversampled and morebits system, where the LMMSE channel estimator for a system with 2 or 3 bits is based on [6]. It can be seen that a system with 2 or 3 bits has better performance than the 1-bit system especially at high SNR. However, the advantage of 1-bit ADCs is that they do not require automatic gain controls (RGC) and linear amplifiers, and hence the corresponding radio frequency chains can be implemented with very low cost and power consumption. 


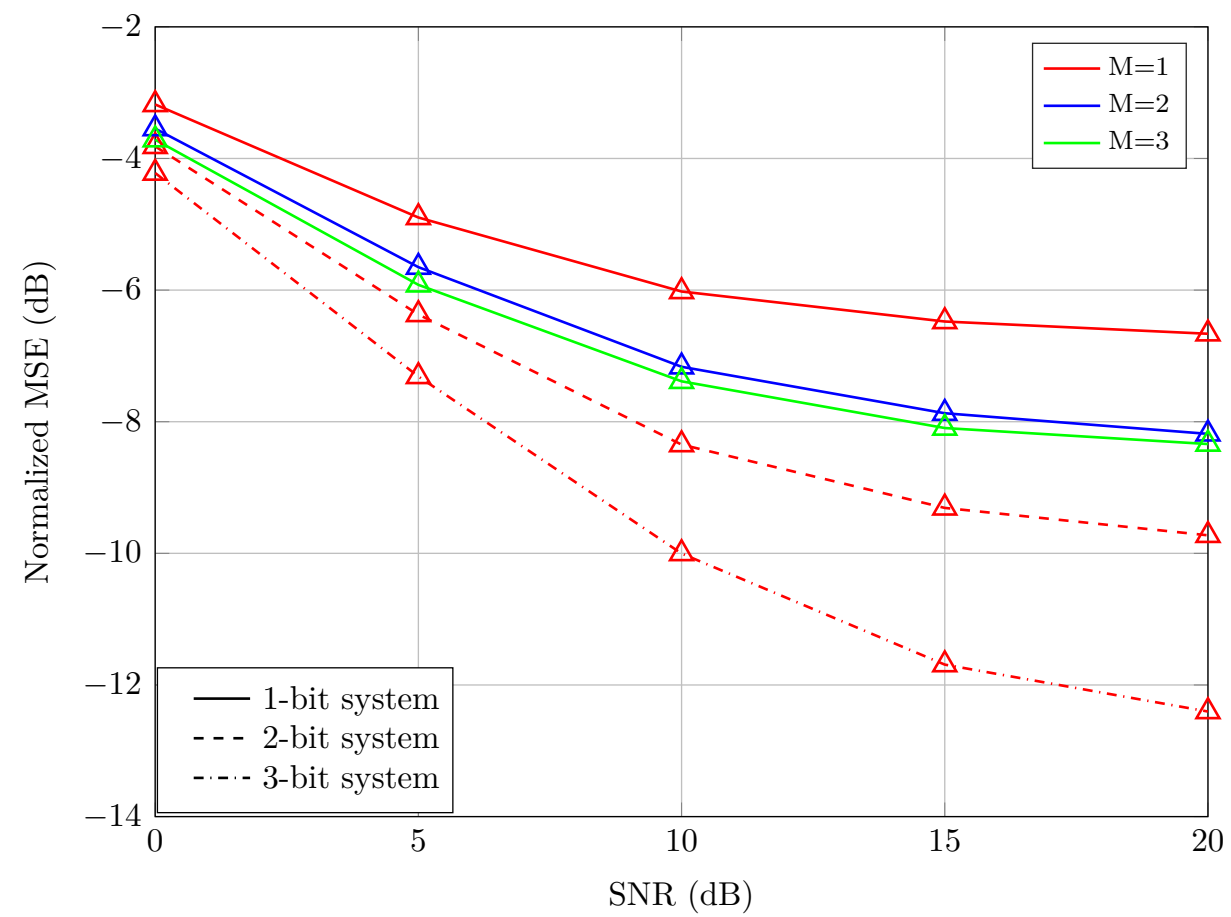

Figure 4.13: Normalized MSE comparisons of LMMSE channel estimator with known $\mathbf{R}_{\mathbf{h}^{\prime}}$ under uncorrelated channel $(|\rho|=0)$.

\section{4}

\section{Signal Detection}

Unlike existing systems in the literature, the received signal cannot be detected from one time instant to another due to the ISI in oversampled systems. The authors in [84] have used ZF and MRC linear receivers for oversampled systems by using all the symbols in one block, which requires a high computational complexity to yield high accuracy. As the block length grows the computational complexities will increase drastically. By contrast, a sliding window based detection technique is proposed, where detection can be processed in a much shorter window range. Different receive filters can be combined with this technique. In particular, LRA-ZF and LRA-LMMSE receive filters for combined use with oversampling and sliding windows.

\subsection{1}

\section{Proposed Sliding Window Based Receiver}

In what follows, an advanced receiver with low complexity is proposed, which is based on the sliding window technique. The oversampled signal at the receiver in each block can be illustrated as Fig. 4.14 (for $M=2$ ).

While performing detection a sliding window is equipped at each receive antenna. The mechanism is shown in Fig. 4.15. To clarify this further, an example is taken where the window length $l_{\text {win }}$ is equal to 3 non-oversampled 


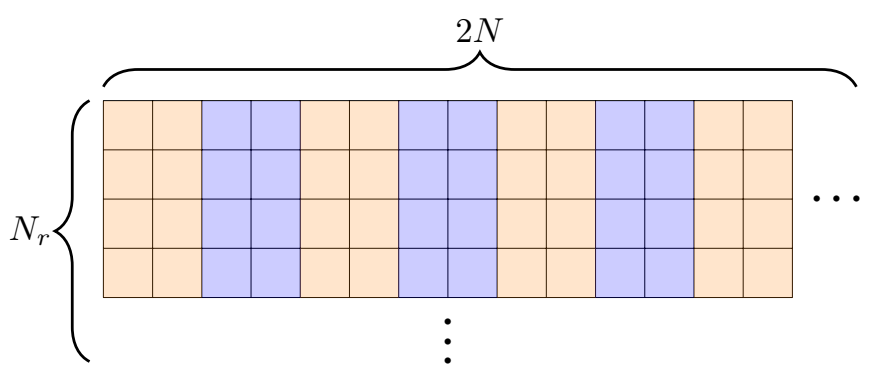

Figure 4.14: Illustration of one block of oversampled signal at the receiver.

symbols. To avoid confusion the first and the last window are not shown in the figure. They are slight different from the normal case and will be described later.

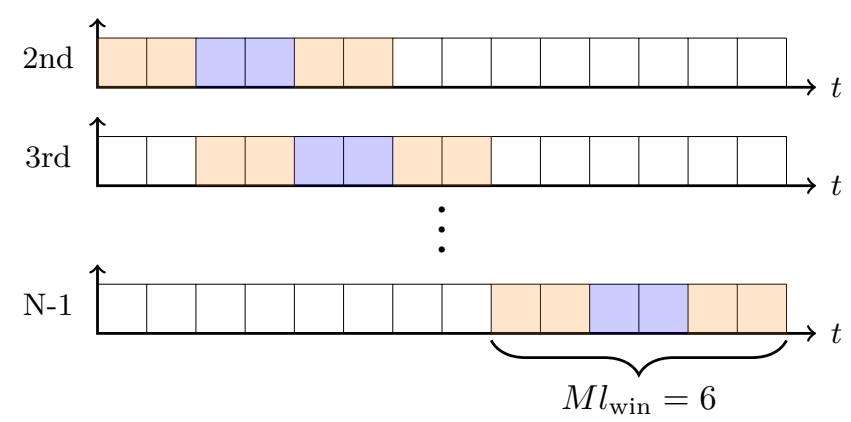

Figure 4.15: Illustration of the sliding window at each receive antenna excluding the first and the last window when $l_{\text {win }}=3$ and $M=2$.

By extracting the signals from (4-8) into each corresponding window, we obtain the quantized signal in each window as

$$
\mathbf{y}_{\mathcal{Q}_{\text {win }}}=\mathcal{Q}\left(\mathbf{y}_{\text {win }}\right)=\mathcal{Q}\left(\mathbf{H}_{\text {win }} \mathbf{x}_{\text {win }}+\mathbf{n}_{\text {win }}\right),
$$

where $\mathbf{x}_{\text {win }}$ is a $l_{\text {win }} N_{t} \times 1$ column vector. $\mathbf{H}_{\text {win }}$ is a $M l_{\text {win }} N_{r} \times l_{\text {win }} N_{t}$ equivalent channel matrix and $\mathbf{n}_{\text {win }} \in \mathbb{C}^{M l_{\text {win }} N_{r} \times 1}$. The soft estimate of $\mathbf{x}_{\text {win }}$ can be calculated as

$$
\tilde{\mathbf{x}}_{\text {win }}^{\prime}=\mathbf{B}^{\prime} \mathbf{y}_{\mathcal{Q}_{\text {win }}},
$$

where $\mathbf{B}^{\prime}$ is the receive filter. The ZF-type filter for the oversampled system [84] with a sliding window is described by

$$
\mathbf{B}_{\mathrm{ZF}}^{\prime}=\left(\mathbf{H}_{\mathrm{win}}^{H} \mathbf{H}_{\mathrm{win}}\right)^{-1} \mathbf{H}_{\mathrm{win}}^{H} .
$$

Note that $\tilde{\mathbf{x}}_{\text {win }}^{\prime}$ is not fully used. There is a decimation process, which extracts the symbols at the time instant zero. One example for $l_{\text {win }}=3$ is denoted as

$$
\mathbf{D}=\mathbf{I}_{N_{t}} \otimes \mathbf{d}=\mathbf{I}_{N_{t}} \otimes\left[\begin{array}{lll}
0 & 1 & 0
\end{array}\right],
$$

then the decimated symbols are 


$$
\tilde{\mathbf{x}}_{\text {win }}=\mathbf{D} \tilde{\mathbf{x}}_{\text {win }}^{\prime}=(\mathbf{D B})^{\prime} \mathbf{y}_{\mathcal{Q}_{\text {win }}}=\mathbf{B} \mathbf{y}_{\mathcal{Q}_{\text {win }}},
$$

where $\mathbf{B}$ is the proposed sliding window based receiver.

Two special cases, the first and the last window of each block, will be detailed in the following. Notice that both the first non-oversampled symbol in the first window and the last non-oversampled symbol in the last window are at the time instant zero, then the decimation vector $\mathbf{d}$ will be changed to

$$
\mathbf{d}_{\text {first }}=\left[\begin{array}{lll}
1 & 0 & 0
\end{array}\right] \text { and } \quad \mathbf{d}_{\text {last }}=\left[\begin{array}{lll}
0 & 0 & 1
\end{array}\right] \text {. }
$$

In a more general case, for a given window length $l_{\text {win }}$ the first $\left(l_{\text {win }}-1\right)$ windows start from the first oversampled symbol and the last 2 windows terminate at the end of each block. The corresponding decimation vector will be similar to (4-68). For the first window the one will be placed in the first position of the vector $\mathbf{d}$. For the second window the one will be placed in the second position and the rest can be done in the same manner. For the normal windows, $\mathbf{d}$ is an all-zeros vector except that the $\left(l_{\text {win }}-1\right)$ th position is 1 .

\subsection{2}

\section{LRA-LMMSE Receive Filter}

According to (4-64), the optimization problem for the LMMSE receive filter is

$$
\mathbf{B}_{\mathrm{LRA}-\mathrm{LMMSE}}^{\prime}=\arg \min _{\mathbf{B}^{\prime}} E\left\{\left\|\mathbf{x}_{\mathrm{win}}-\mathbf{B}^{\prime} \mathbf{y}_{\mathcal{Q}_{\text {win }}}\right\|_{2}^{2}\right\},
$$

and the solution is

$$
\mathbf{B}_{\text {LRA-LMMSE }}^{\prime}=\left(\mathbf{C}_{\mathbf{y}_{\mathcal{Q}_{\text {win }}}^{-1}}^{-\mathbf{C}_{\mathbf{y}_{\mathcal{Q}_{\text {win }}} \mathbf{x}_{\text {win }}}}\right)^{H} .
$$

In (4-70) the covariance matrix $\mathbf{C}_{\mathbf{y}_{\mathcal{Q}_{\text {win }}}}$ is calculated as [33]

$$
\mathbf{C}_{\mathbf{y}_{\mathcal{Q}_{\text {win }}}}=\frac{2}{\pi}\left(\sin ^{-1}\left(\mathbf{K} \Re\left\{\mathbf{C}_{\mathbf{y}_{\text {win }}}\right\} \mathbf{K}\right)+j \sin ^{-1}\left(\mathbf{K} \mathfrak{I}\left\{\mathbf{C}_{\mathbf{y}_{\text {win }}}\right\} \mathbf{K}\right)\right)
$$

and the cross-correlation vector $\mathbf{C}_{\mathbf{y}_{\mathcal{Q}_{\text {win }}} \mathbf{x}_{\text {win }}}$ is given by [32]

$$
\mathbf{C}_{\mathbf{y}_{\mathcal{Q}_{\text {win }}} \mathbf{x}_{\text {win }}}=\sqrt{\frac{2}{\pi}} \mathbf{K H}_{\text {win }}, \quad \text { with } \quad \mathbf{K}=\operatorname{diag}\left(\mathbf{C}_{\mathbf{y}_{\text {win }}}\right)^{-\frac{1}{2}}
$$

where $\mathbf{C}_{\mathbf{y}_{\text {win }}}$ is the covariance matrix of the unquantized data vector $\mathbf{y}_{\text {win }}$, which leads to the following result

$$
\begin{aligned}
\mathbf{C}_{\mathbf{y}_{\text {win }}} & =E\left\{\left(\mathbf{H}_{\text {win }} \mathbf{x}_{\text {win }}+\mathbf{n}_{\text {win }}\right)\left(\mathbf{H}_{\text {win }} \mathbf{x}_{\text {win }}+\mathbf{n}_{\text {win }}\right)^{H}\right\} \\
& =\mathbf{H}_{\text {win }} \mathbf{H}_{\text {win }}^{H}+\mathbf{C}_{n_{\text {win }}}
\end{aligned}
$$

with $\mathbf{C}_{n_{\text {win }}}=\sigma_{n}^{2} \mathbf{I}_{N_{r}} \otimes \mathbf{R}_{\text {win }} \mathbf{R}_{\text {win }}^{H}$. $\mathbf{R}_{\text {win }}$ represents $\mathbf{R}$ in each window. Unlike prior work on LRA-LMMSE receive filters, we obtain expressions for a system with oversampling and sliding windows. 


\subsection{3}

\section{LRA-ZF Receive Filter}

Recall the system model in (4-63), based on the Bussgang decomposition it can be written as

$$
\mathbf{y}_{\mathcal{Q}_{\text {win }}}=\mathbf{A y}_{\text {win }}+\mathbf{n}_{q},
$$

where $\mathbf{A}$ is the linear operator that is chosen to make $\mathbf{n}_{q}$ uncorrelated with $\mathbf{y}_{\text {win }}$. The vector $\mathbf{n}_{q}$ is the statistically equivalent quantization noise with covariance matrix $\mathbf{C}_{\mathbf{n}_{q}}=\mathbf{C}_{\mathbf{y}_{\mathcal{Q}_{\text {win }}}}-\mathbf{A C}_{\mathbf{y}_{\text {win }}} \mathbf{A}$. The matrix $\mathbf{A}$ is calculated as

$$
\mathbf{A}=\mathbf{C}_{\mathbf{y}_{\text {win }} \mathbf{y}_{\mathcal{Q}_{\text {win }}}}^{H} \mathbf{C}_{\mathbf{y}_{\text {win }}}^{-1}=\sqrt{\frac{2}{\pi}} \operatorname{diag}\left(\mathbf{C}_{\mathbf{y}_{\text {win }}}\right)^{-\frac{1}{2}} .
$$

The ZF filter minimizes the mean square error (MSE) as

$$
\mathbf{B}_{\mathrm{LRA}-\mathrm{ZF}}^{\prime}=\arg \min _{\mathbf{B}^{\prime}} E\left\{\left\|\mathbf{B}^{\prime}\left(\mathbf{A} \mathbf{n}_{\mathrm{win}}+\mathbf{n}_{q}\right)\right\|_{2}^{2}\right\} \quad \text { s.t.: } \mathbf{B}^{\prime} \mathbf{A} \mathbf{H}_{\mathrm{win}}=\mathbf{I}_{l_{\mathrm{win}} N_{t}} .
$$

With the Lagrangian multiplier, we obtain

$$
\mathbf{B}_{\mathrm{LRA}-\mathrm{ZF}}^{\prime}=\left(\mathbf{H}_{\mathrm{win}}^{H} \mathrm{AC}_{n}^{-1} \mathbf{A} \mathbf{H}_{\mathrm{win}}\right)^{-1} \mathbf{H}_{\mathrm{win}}^{H} \mathbf{A C}_{n}^{-1}
$$

where $\mathbf{C}_{n}=\mathbf{A C}_{n_{\text {win }}} \mathbf{A}+\mathbf{C}_{n_{q}}$.

\subsection{4}

\section{Numerical Results}

In this subsection, the proposed sliding window based receiver is examined in terms of the symbol error rate (SER). The modulation scheme is QPSK. The channel is assumed to experience block fading, where each block contains 100 symbols. The $m(t)$ and $p(t)$ are normalized RRC pulse filter with a rolloff factor of 0.8. The simulation based SER performance plots are obtained by taking the average of 300 channel realizations and by defining the SNR as $10 \log \left(\frac{N_{t} \sigma_{x}^{2}}{\sigma_{n}^{2}}\right)$.

The SER performance of LRA-ZF and LRA-LMMSE receive filter based sliding window techniques are shown in Fig. 4.16 and Fig. 4.17, where the performances of different oversampling rates are compared. It can be observed that oversampling by 2 results in a large SNR advantage as compared to the case where no oversampling is performed. The SER curve for an oversampling rate of 4 is also provided, which has about $2 \mathrm{~dB}$ SNR gain as compared to the case of $M=2$. Moreover, the performances of large window systems $\left(l_{\text {win }}=25\right)$ are also shown in the figures, where they have very similar performances with short window systems. One notable observation is that at high SNR the SER performance degrades when the system is oversampled above the factor of two and detected with window length of one. One possible explanation 
for this performance degradation is that at high SNR the dominant noise is the interference from adjacent symbols, but window length of one has not considered this interference which results in large detection error.

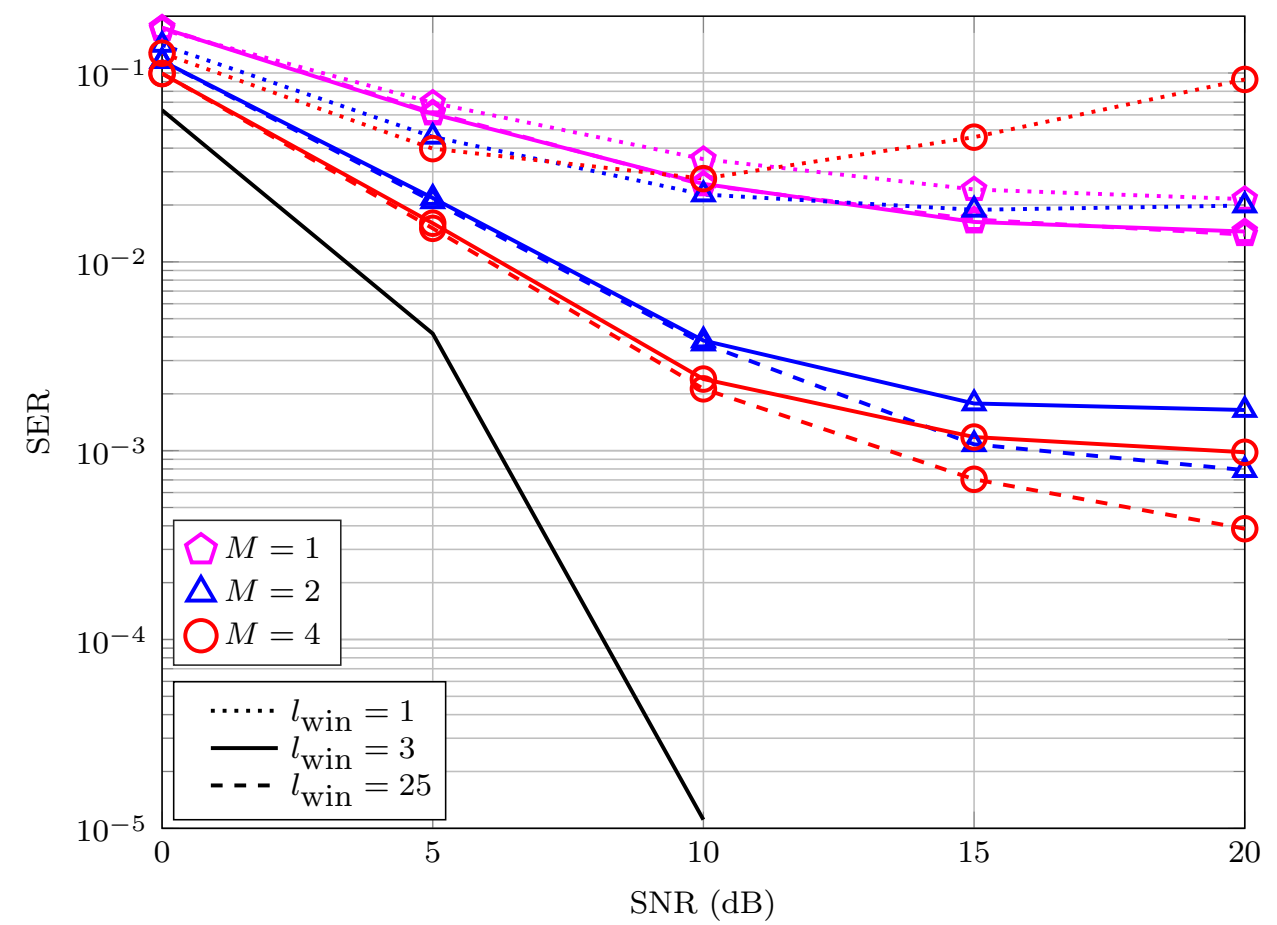

Figure 4.16: $N_{t}=12$ and $N_{r}=64$. SER performance comparison with LRA-ZF receiver, where the black line represents the non-oversampled full-resolution system with ZF receive filter.

Regarding the computational complexity of the analyzed schemes using the ZF filter, the proposed sliding window technique $\left(\mathcal{O}\left(N_{t}^{3} l_{\text {win }}^{3} N\right)\right)$ has a much lower cost than that of the non-windowed systems $\left(\mathcal{O}\left(N_{t}^{3} N^{3}\right)\right)$ [84], when $N$ is large enough. From Fig. 4.18, the SER performance will maintain the same level after $l_{\text {win }}=4$. This indicates that the performance of the nonwindowed systems are the same as those of systems with a large window length $\left(l_{\text {win }}=25\right)$.

\section{5}

\section{Summary}

In this chapter, oversampling based low-resolution aware channel estimators have been proposed for uplink single-cell large-scale MIMO systems with 1-bit ADCs employed at the receiver. The Bussgang decomposition is used to derive linear channel estimators based on different criteria. With oversampling in such systems, it is observed that we can achieve obvious advantage compared to the non-oversampled system in terms of the normalized MSE. Moreover, the LMS adaptive technique used for channel estimation can largely reduce the computational cost and has almost the same accuracy as the LRA- 


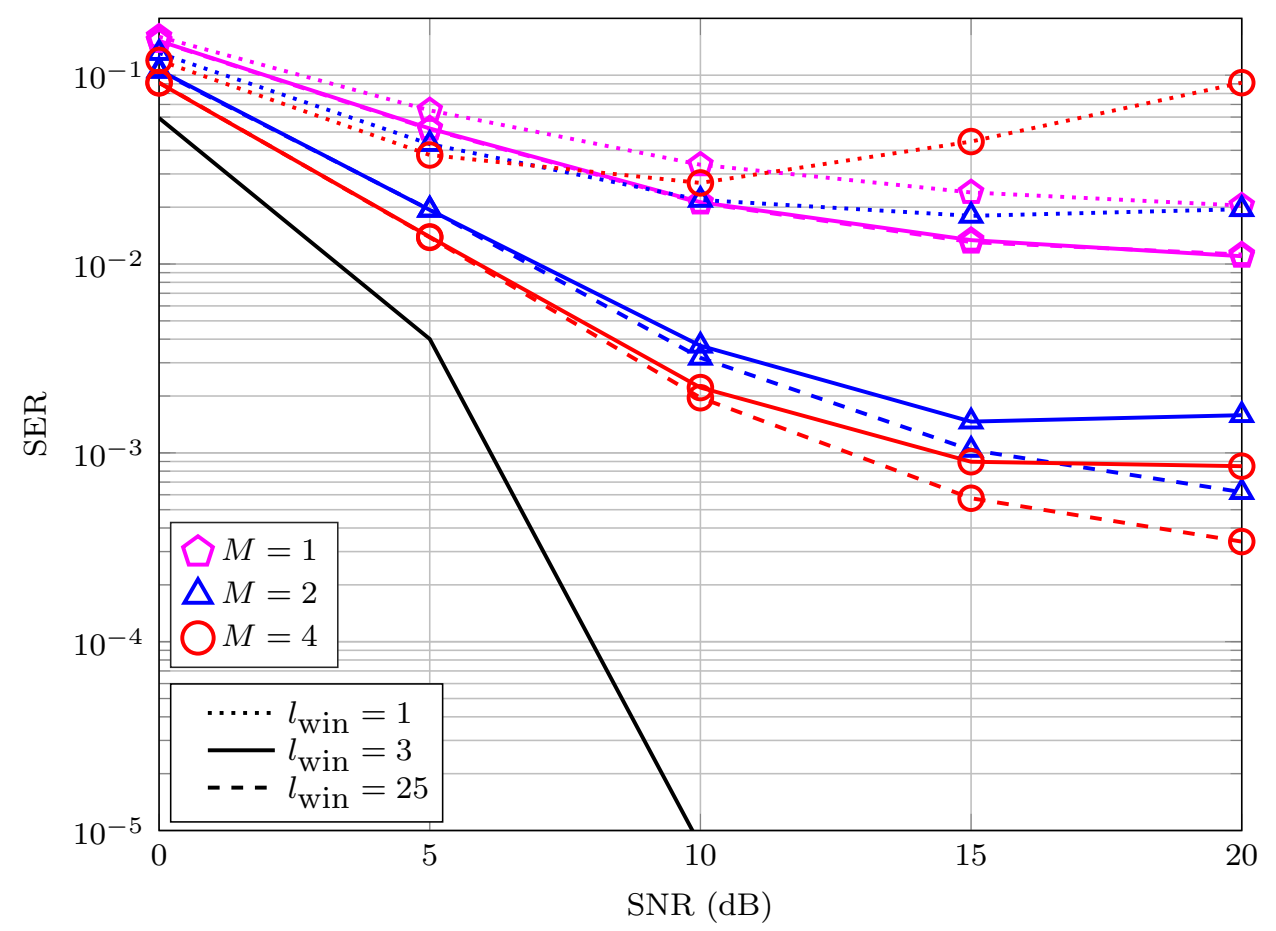

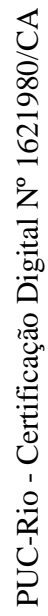

Figure 4.17: $N_{t}=12$ and $N_{r}=64$. SER performance comparison with LRA-LMMSE receiver, where the black line represents the non-oversampled full-resolution system with LMMSE receive filter.

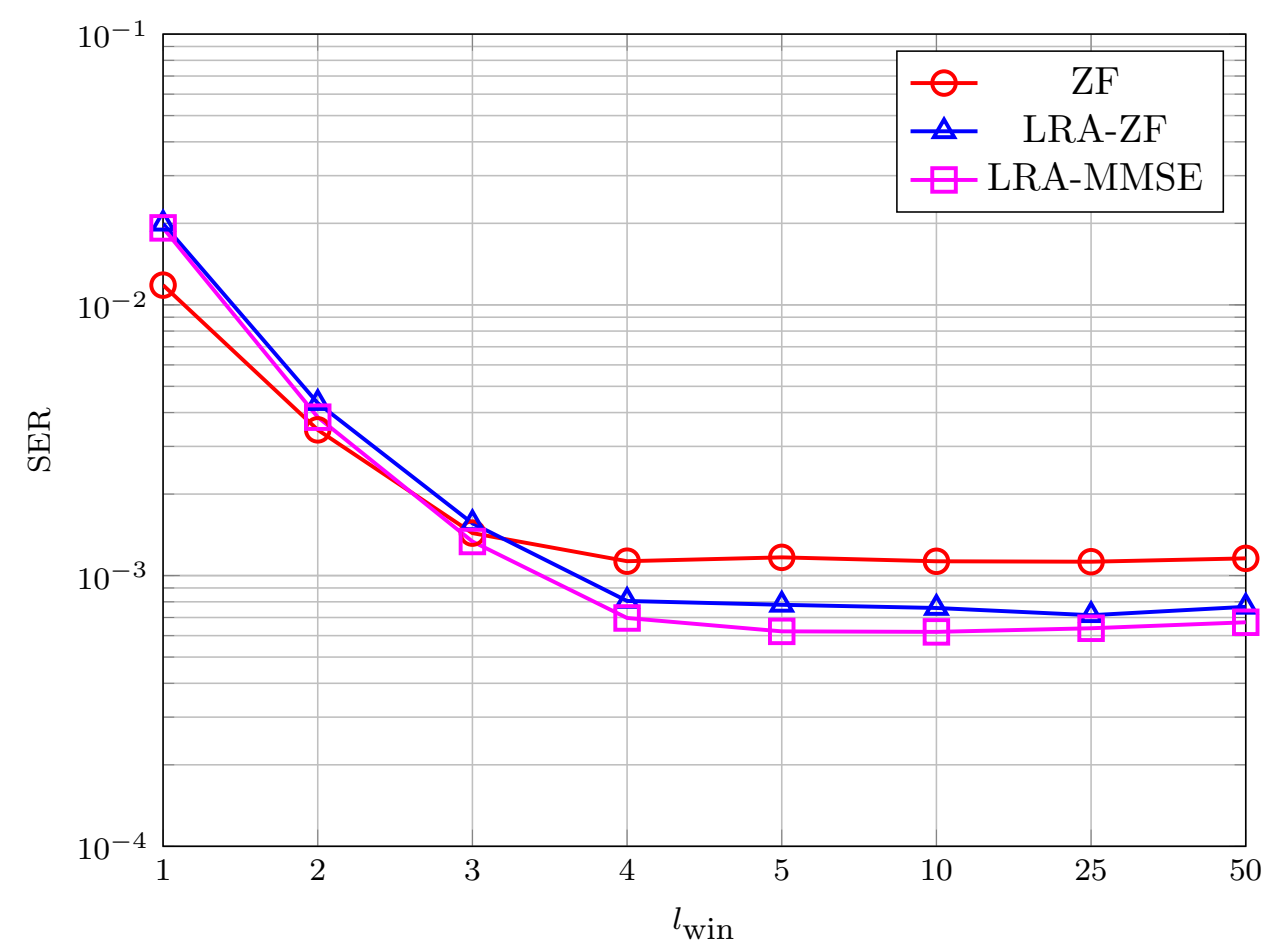

Figure 4.18: $N_{t}=12$ and $N_{r}=64$. SER performance comparison among different window lengths, $M=2$ and SNR $=20 d B$. 
LMMSE channel estimator at low SNR, which is important to ensure low computational complexity and for hardware implementation. In addition, Bayesian and general CRBs on MSE are derived, which give theoretical limits on the performance of the channel estimators. Furthermore, an adaptive technique to estimate the auto-correlation of the channel vector is proposed, which is important for practical use.

In order to achieve low signal processing complexity, the sliding window based receiver for 1-bit oversampled systems has been proposed. The simulation results have shown that the system with short window length can achieve the same SER performance as the system considering the whole transmission block. The sliding window based LRA-ZF and LRA-LMMSE receive filters have also been proposed. It can be seen that a gain of $5 \mathrm{~dB}$ in SNR for the same SER with oversampling of two is achieved over the standard non-oversampled system. 


\section{5 \\ Dynamic-oversampling-rate based System Design}

In the previous chapter, we have seen that oversampling can largely improve the system performance including the MSE of channel estimates and the SER in synchronous mode. For example, the work in [76] has proposed several channel estimation algorithms for 1-bit systems with uniform oversampling. The error floor of MSE at high SNR has been obviously lowered. The work in [67] has shown that oversampling can provide a gain in signal-to-noise ratio (SNR) of about $5 \mathrm{~dB}$ for the same symbol error rate (SER) and achievable rate with a linear zero forcing (ZF) receiver. Analytical bounds on the SER and achievable rate have also been given.

In this chapter, the oversampling technique is investigated in asynchronous large-scale MU-MIMO systems with 1-bit ADCs at the receiver. However, different from uniform oversampling, a dynamic oversampling technique is first proposed in the new system. In the proposed system two rates are introduced, initial sampling rate and signal processing rate. The received signal is initially oversampled at a higher rate and then processed by dimension reduction matrices, which either combine or select samples prior to further signal processing. The criterion of which samples are combined or selected is based on how much information contributes to the desired system designs, namely, maximizing the sum rate or minimizing the MSE of the detected symbols. Simulation results show that the systems with proposed dynamic oversampling outperform the uniformly oversampled system in terms of the achievable sum rate and SER performance without increasing more computational cost.

In section 5.1, the system model of asynchronous 1-bit MU-MIMO is firstly presented. The design criteria of dynamic oversampling are detailed in section 5.2 and section 5.3 introduces the dimension reduction algorithms. Section 5.4 analyzes the proposed scheme based on its convergence and computational complexity. Section 5.5 illustrates and discusses the numerical results. 


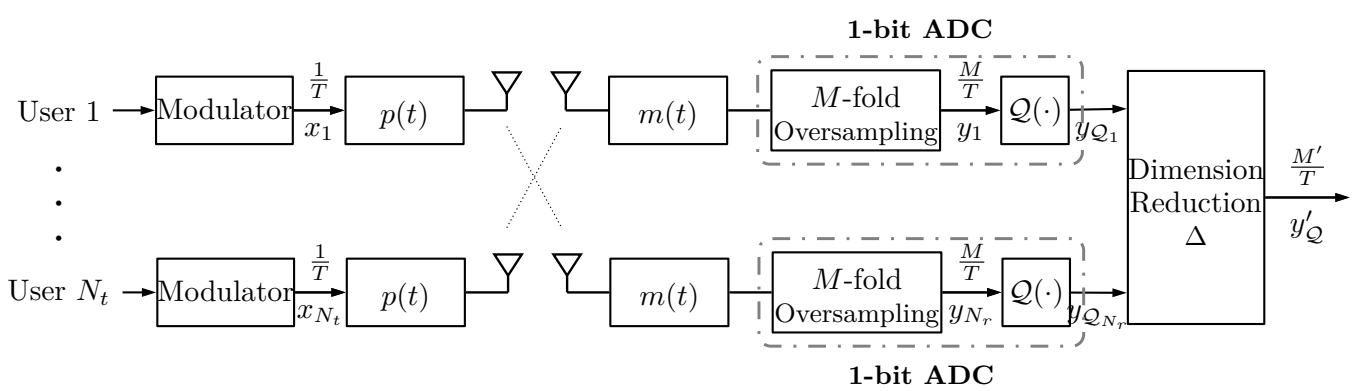

Figure 5.1: MU-MIMO with 1-bit ADCs and dynamic oversampling at the receiver.

\section{1}

\section{System Model}

The overall system model is illustrated with a block diagram in Fig. 5.1 , where at the BS the system is firstly oversampled by a factor of $M$ (initial sampling rate) and downsampled to a factor of $M^{\prime}$ (signal processing rate). The received oversampled signal $\mathbf{y}$ for the single-cell uplink large-scale multiple-antenna system with $N_{t}$ single-antenna users and $N_{r}$ receive antennas $\left(N_{r} \gg N_{t}\right)$ is written as

$$
\mathbf{y}=\mathbf{H} \mathbf{x}+\mathbf{n} .
$$

The vector $\mathbf{x} \in \mathbb{C}^{N N_{t} \times 1}$ contains all transmitted symbols within one block and is arranged as

$$
\mathbf{x}=\left[\begin{array}{llllll}
x_{1,1} & \cdots & x_{N, 1} & x_{1,2} & \cdots & x_{N, N_{t}}
\end{array}\right]^{T},
$$

where $x_{i, j}$ corresponds to the transmitted symbol of terminal $j$ at time instant $i$ and is IID with unit power $E\left[\left|x_{i, j}\right|^{2}\right]=1$. The vector $\mathbf{n} \in \mathbb{C}^{M N N_{r} \times 1}$ is the filtered oversampled noise expressed by

$$
\mathbf{n}=\left(\mathbf{I}_{N_{r}} \otimes \mathbf{G}\right) \mathbf{w}
$$

where $\mathbf{w} \sim \mathcal{C N}\left(\mathbf{0}_{3 M N N_{r}}, \sigma_{n}^{2} \mathbf{I}_{3 M N N_{r}}\right)^{1}$ contains IID complex Gaussian random variables with zero mean and variance $\sigma_{n}^{2}$. The matrix $\mathbf{G} \in \mathbb{R}^{M N \times 3 M N}$ is a Toeplitz matrix described by $(5-4)$, where $m(t)$ represents the impulse response of receive filter at the time instant $t$

$\mathbf{G}=\left[\begin{array}{ccccccc}m(-N T) & m\left(-N T+\frac{1}{M} T\right) & \ldots & m(N T) & 0 & \ldots & 0 \\ 0 & m(-N T) & \ldots & m\left(N T-\frac{1}{M} T\right) & m(N T) & \ldots & 0 \\ \vdots & \vdots & \ddots & \vdots & \vdots & \ddots & \vdots \\ 0 & 0 & \ldots & m(-N T) & m\left(-N T+\frac{1}{M} T\right) & \ldots & m(N T)\end{array}\right]$

${ }^{1}$ Note that the noise samples are described such that each entry of $\mathbf{n}$ has the same statistical properties. Since the receive filter $m(t)$ has a length of $2 M N+1$ samples, $3 M N$ unfiltered noise samples in the noise vector $\mathbf{w}$ need to be considered for the description of an interval of $M N$ samples of the filtered noise $\mathbf{n}$. 
$T$ represents one symbol period. In the block scheme, the initial sampling rate is $M$ and the signal processing rate is $M^{\prime}$, where $M \geq M^{\prime}$. The equivalent channel matrix $\mathbf{H}$ is

$$
\mathbf{H}=\left(\mathbf{H}^{\prime} \otimes \mathbf{I}_{M N}\right) \operatorname{blkdiag}\left(\left[\mathbf{Z}_{1}, \ldots, \mathbf{Z}_{N_{t}}\right]\right)\left(\mathbf{I}_{N N_{t}} \otimes \mathbf{u}\right),
$$

where $\mathbf{H}^{\prime} \in \mathbb{C}^{N_{r} \times N_{t}}$ is the standard channel matrix. The vector $\mathbf{u}$ is employed as an oversampling operator defined as the vector $\mathbf{u}$ with the size of $M \times 1$

$$
\mathbf{u}=\left[\begin{array}{llll}
0 & \cdots & 0 & 1
\end{array}\right]^{T} .
$$

Similar to $\mathbf{G}$, the Toeplitz matrix $\mathbf{Z}_{n_{t}} \in \mathbb{R}^{M N \times M N}$ contains the impulse response of $z(t)$ at different time instants, where $z(t)$ is the convolution of transmit filter $p(t)$ and receive filter $m(t)$, and is given by

$$
\mathbf{Z}_{n_{t}}=\left[\begin{array}{cccc}
z\left(n_{n_{t}}^{d}\right) & z\left(n_{n_{t}}^{d}+\frac{T}{M}\right) & \ldots & z\left(n_{n_{t}}^{d}+N T-\frac{1}{M} T\right) \\
z\left(n_{n_{t}}^{d}-\frac{T}{M}\right) & z\left(n_{n_{t}}^{d}\right) & \ldots & z\left(n_{n_{t}}^{d}+N T-\frac{2}{M} T\right) \\
\vdots & \vdots & \ddots & \vdots \\
z\left(n_{n_{t}}^{d}-N T+\frac{1}{M} T\right) & z\left(n_{n_{t}}^{d}-N T+\frac{2}{M} T\right) & \ldots & z\left(n_{n_{t}}^{d}\right)
\end{array}\right] .
$$

In practical communication systems, the transmission delay is unavoidable due to the different transmission paths of the users to the BS. In this chapter, an asynchronous system is considered by assuming the terminal $n_{t}$ sends its signal to the BS delayed by the time $n_{n_{t}}^{d}$.

Let $\mathcal{Q}(\cdot)$ represents the 1-bit quantization at the receiver, the resulting quantized signal $\mathbf{y}_{\mathcal{Q}}$ is

$$
\mathbf{y}_{\mathcal{Q}}=\mathcal{Q}(\mathbf{y})=\mathcal{Q}(\Re\{\mathbf{y}\})+j \mathcal{Q}(\mathfrak{I}\{\mathbf{y}\}),
$$

where $\mathfrak{R}\{\cdot\}$ and $\mathfrak{I}\{\cdot\}$ get the real and imaginary part, respectively. They are quantized element-wisely to $\{ \pm 1\}$ and scaled to $\left\{ \pm \frac{1}{\sqrt{2}}\right\}$ based on the sign.

The Bussgang theorem [32] implies that the output of the nonlinear quantizer can be decomposed into a desired signal component and an uncorrelated distortion noise $\mathbf{n}_{q}$

$$
\mathbf{y}_{\mathcal{Q}}=\mathbf{A y}+\mathbf{n}_{q},
$$

where $\mathbf{A} \in \mathbb{R}^{M N N_{r} \times M N N_{r}}$ is the linear operator chosen independently from $\mathbf{y}$, obtained as

$$
\mathbf{A}=\mathbf{C}_{\mathbf{y y}_{\mathcal{Q}}} \mathbf{C}_{\mathbf{y}}^{-1}
$$

and the distortion $\mathbf{n}_{q}$ has the following covariance matrix

$$
\mathbf{C}_{\mathbf{n}_{q}}=\mathbf{C}_{\mathbf{y}_{\mathcal{Q}}}-\mathbf{A C}_{\mathbf{y}} \mathbf{A}
$$

The involved $\mathbf{C}_{\mathbf{y y}_{\mathcal{Q}}}$ denotes the cross-correlation between the received signal $\mathbf{y}$ and its quantized form $\mathbf{y}_{\mathcal{Q}}$ 


$$
\mathbf{C}_{\mathbf{y y}_{\mathcal{Q}}}=\sqrt{\frac{2}{\pi}} \mathbf{K C}_{\mathbf{y}}, \quad \text { with } \quad \mathbf{K}=\operatorname{diag}\left(\mathbf{C}_{\mathbf{y}}\right)^{-\frac{1}{2}}
$$

and the auto-correlation matrix $\mathbf{C}_{\mathbf{y}_{\mathcal{Q}}}$ is obtained through the arcsin law [33]

$$
\mathbf{C}_{\mathbf{y}_{\mathcal{Q}}}=\frac{2}{\pi}\left(\sin ^{-1}\left(\mathbf{K C}_{\mathbf{y}}^{R} \mathbf{K}\right)+j \sin ^{-1}\left(\mathbf{K C}_{\mathbf{y}}^{I} \mathbf{K}\right)\right)
$$

where $\mathbf{C}_{\mathbf{y}}$ is calculated as

$$
\begin{aligned}
\mathbf{C}_{\mathbf{y}} & =\mathbf{H} \mathbf{H}^{H}+\mathbf{C}_{\mathbf{n}} \\
& =\mathbf{H H}^{H}+\sigma_{n}^{2}\left(\mathbf{I}_{N_{r}} \otimes \mathbf{G G}^{H}\right) .
\end{aligned}
$$

Based on this decomposition, the signal after the 1-bit quantizer can be written in the following form

$$
\begin{aligned}
\mathbf{y}_{\mathcal{Q}} & =\mathbf{A y}+\mathbf{n}_{q} \\
& =\mathbf{A H x}+\mathbf{A n}+\mathbf{n}_{q} .
\end{aligned}
$$

\section{2}

\section{System Design with Dynamic Oversampling}

Unlike the conventional uniform oversampling technique used in most of the applications, an advanced oversampling technique is devised, named dynamic oversampling, where the system is initially oversampled at a higher rate $M$ and only few samples are selected and further processed. The uniform oversampling can be treated as a special case of dynamic oversampling when $M=M^{\prime}$. In this section, dynamic oversampled systems are derived based on two different design strategies, maximizing the sum rate or minimizing the MSE of detected symbols.

\section{2 .1}

\section{Sum Rate based System Design}

Let us consider $\mathbf{n}^{\prime}=\mathbf{A n}+\mathbf{n}_{q}$ in (5-15) and assume it is Gaussian distributed with the covariance matrix

$$
\mathbf{C}_{\mathbf{n}^{\prime}}=\mathbf{A} \mathbf{C}_{\mathbf{n}} \mathbf{A}+\mathbf{C}_{\mathbf{n}_{q}}
$$

the uplink sum rate lower bound ${ }^{2}$ is then given by

$$
C_{L B}=E\left\{\frac{1}{N} \log _{2} \operatorname{det}\left(\mathbf{I}_{M N N_{r}}+\mathbf{A} \mathbf{H} \mathbf{H}^{H} \mathbf{A C}_{\mathbf{n}^{\prime}}^{-1}\right)\right\}
$$

which can be simplified as

$$
C_{L B}=E\left\{\frac{1}{N} \log _{2} \operatorname{det}\left(\mathbf{C}_{\mathbf{y}_{\mathcal{Q}}} \mathbf{C}_{\mathbf{n}^{\prime}}^{-1}\right)\right\} .
$$

${ }^{2}$ Note that the actual sum rate is difficult to calculate due to the unknown characteristic of the distortion noise $\mathbf{n}_{q}$. Similar to the work in [77], we assume $\mathbf{n}^{\prime}$ is Gaussian distributed. This method minimizes the actual mutual information but simplifies the analysis. 
Assuming that the dimension reduction operation can be mathematically described as a linear transformation with the matrix $\boldsymbol{\Delta}[78]$, the received signal is then reduced to

$$
\mathbf{y}_{\mathcal{Q}}^{\prime}=\Delta \mathbf{y}_{\mathcal{Q}}
$$

where $\Delta$ has the size of $M^{\prime} N N_{r} \times M N N_{r}$. The sum rate lower bound for the dynamic oversampled system is

$$
C_{L B}=E\left\{\frac{1}{N} \log _{2} \operatorname{det}\left(\Delta \mathbf{C}_{\mathbf{y}_{\mathcal{Q}}} \boldsymbol{\Delta}^{H}\left(\boldsymbol{\Delta} \mathbf{C}_{\mathbf{n}^{\prime}} \boldsymbol{\Delta}^{H}\right)^{-1}\right)\right\} .
$$

The optimization problem that corresponds to the design of the optimal $\boldsymbol{\Delta}^{\mathrm{opt}}$ that can obtain the highest achievable sum rate is described as

$$
\boldsymbol{\Delta}^{\mathrm{opt}}=\arg \max _{\boldsymbol{\Delta}} \log _{2} \operatorname{det}\left(\boldsymbol{\Delta} \mathbf{C}_{\mathbf{y}_{\mathcal{Q}}} \boldsymbol{\Delta}^{H}\left(\boldsymbol{\Delta} \mathbf{C}_{\mathbf{n}^{\prime}} \boldsymbol{\Delta}^{H}\right)^{-1}\right) .
$$

Since the determinant is a log-concave function [79] and with the properties of the determinant, (5-21) is simplified as

$$
\boldsymbol{\Delta}^{\mathrm{opt}}=\arg \max _{\boldsymbol{\Delta}} \frac{\operatorname{det}\left(\Delta \mathbf{C}_{\mathbf{y}_{\mathcal{Q}}} \boldsymbol{\Delta}^{H}\right)}{\operatorname{det}\left(\boldsymbol{\Delta} \mathbf{C}_{\mathbf{n}^{\prime}} \boldsymbol{\Delta}^{H}\right)} .
$$

According to [80], the solution of (5-22) is equivalent to the solution of following optimization problem

$$
\boldsymbol{\Delta}^{\mathrm{opt}}=\arg \max _{\boldsymbol{\Delta}} \operatorname{Tr}\left(\boldsymbol{\Delta} \mathbf{C}_{\mathbf{y}_{\mathcal{Q}}} \boldsymbol{\Delta}^{H}\left(\boldsymbol{\Delta} \mathbf{C}_{\mathbf{n}^{\prime}} \boldsymbol{\Delta}^{H}\right)^{-1}\right),
$$

which will be solved in section 5.3 .

\section{2 .2}

\section{Mean Square Error based System Design}

In this system, the reduction matrix should be designed to obtain the least MSE of the detected symbols. In order to reduce the computational cost while performing detection, the sliding window technique is applied.

Similar to (5-19), the dimension reduction operation in each window can be mathematically described as

$$
\mathbf{y}_{\mathcal{Q}_{\text {win }}}^{\prime}=\boldsymbol{\Delta}_{\text {win }} \mathbf{y}_{\mathcal{Q}_{\text {win }}}
$$

where $\boldsymbol{\Delta}_{\text {win }}$ is the reduction matrix with the size of $M^{\prime} l N_{r} \times M l N_{r}$ for each window and $l$ denotes the window length. $\mathbf{y}_{\mathcal{Q}_{\text {win }}} \in \mathbb{C}^{M l N_{r} \times 1}$ represents the received signal in each window. The optimization problem that leads to the optimal linear detector is formulated as

$$
\mathbf{W}_{\text {LRA-MMSE }}=\arg \min _{\mathbf{W}} E\left\{\left\|\mathbf{x}_{\text {win }}-\mathbf{W}^{H} \mathbf{y}_{\mathcal{Q}_{\text {win }}}^{\prime}\right\|_{2}^{2}\right\},
$$

where $\mathbf{W} \in \mathbb{C}^{M^{\prime} l N_{r} \times l N_{t}}$ and the solution is 


$$
\begin{aligned}
\mathbf{W}_{\text {LRA-MMSE }} & =\mathbf{C}_{\mathbf{y}_{\mathcal{Q}_{\text {win }}^{\prime}}^{-1}}^{-1} \mathbf{C}_{\mathbf{y}_{\mathcal{Q}_{\text {win }}}^{\prime} \mathbf{x}_{\text {win }}} \\
& =\left(\boldsymbol{\Delta}_{\text {win }} \mathbf{C}_{\mathbf{y}_{\mathcal{Q}_{\text {win }}}} \boldsymbol{\Delta}_{\text {win }}^{H}\right)^{-1} \boldsymbol{\Delta}_{\text {win }} \mathbf{C}_{\mathbf{y}_{\mathcal{Q}_{\text {win }}} \mathbf{x}_{\text {win }}} .
\end{aligned}
$$

According to (5-12) and (5-13), the involved covariance matrix $\mathbf{C}_{\mathbf{y}_{\mathcal{Q}_{\mathbf{w i n}}}}$ and the cross-correlation matrix $\mathbf{C}_{\mathbf{y}_{\mathcal{Q}_{\text {win }}} \mathbf{x}_{\text {win }}}$ are calculated as

$$
\begin{gathered}
\mathbf{C}_{\mathbf{y}_{\mathcal{Q}_{\text {win }}}}=\frac{2}{\pi}\left(\sin ^{-1}\left(\mathbf{K}_{\text {win }} \mathbf{C}_{\mathbf{y}_{\text {win }}}^{R} \mathbf{K}_{\text {win }}\right)+j \sin ^{-1}\left(\mathbf{K}_{\text {win }} \mathbf{C}_{\mathbf{y}_{\text {win }}}^{I} \mathbf{K}_{\text {win }}\right)\right) \\
\mathbf{C}_{\mathbf{y}_{\mathcal{Q}_{\text {win }}} \mathbf{x}_{\text {win }}}=\sqrt{\frac{2}{\pi}} \mathbf{K}_{\text {win }} \mathbf{H}_{\text {win }}, \text { with } \mathbf{K}_{\text {win }}=\operatorname{diag}\left(\mathbf{C}_{\mathbf{y}_{\text {win }}}\right)^{-\frac{1}{2}}
\end{gathered}
$$

The calculation of $\boldsymbol{\Delta}_{\text {win }}$ leads to the following optimization problem

$$
\boldsymbol{\Delta}_{\text {win }}^{\mathrm{opt}}=\arg \min _{\boldsymbol{\Delta}_{\text {win }}} E\left\{\left\|\mathbf{x}_{\text {win }}-\mathbf{W}_{\text {LRA-MMSE }}^{H} \mathbf{y}_{\mathcal{Q}_{\text {win }}}^{\prime}\right\|_{2}^{2}\right\} .
$$

Inserting (5-26) into (5-29) and expanding the terms, we obtain

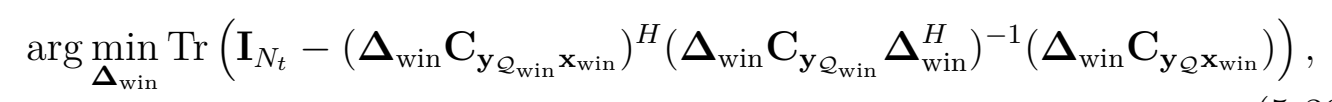

which can be further simplified as

$$
\boldsymbol{\Delta}_{\text {win }}^{\text {opt }}=\arg \max _{\boldsymbol{\Delta}_{\text {win }}} \operatorname{Tr}\left(\boldsymbol{\Delta}_{\text {win }} \mathbf{C}_{\mathbf{y}_{\mathcal{Q}_{\text {win }}} \mathbf{x}_{\text {win }}} \mathbf{C}_{\mathbf{y}_{\mathcal{Q}_{\text {win }}}^{H} \mathbf{x}_{\text {win }}} \boldsymbol{\Delta}_{\text {win }}^{H}\left(\boldsymbol{\Delta}_{\text {win }} \mathbf{C}_{\mathbf{y}_{\mathcal{Q}_{\text {win }}}} \boldsymbol{\Delta}_{\text {win }}^{H}\right)^{-1}\right)
$$

with

$$
\mathbf{C}_{\mathbf{y}_{\mathcal{Q}_{\text {win }}} \mathbf{x}_{\text {win }}} \mathbf{C}_{\mathbf{y}_{\mathcal{Q}_{\text {win }}}^{H} \mathbf{x}_{\text {win }}}^{H}=\frac{2}{\pi} \mathbf{K}_{\text {win }} \mathbf{H}_{\text {win }} \mathbf{H}_{\text {win }}^{H} \mathbf{K}_{\text {win }} .
$$

After obtaining the optimal reduction matrix $\boldsymbol{\Delta}_{\text {win }}^{\text {opt }}$, which will be illustrated in section 5.3, the sliding-window based LRA-MMSE detector for the dynamic oversampled systems is then given by

$$
\mathbf{W}_{\text {LRA-MMSE }}^{\text {opt }}=\left(\boldsymbol{\Delta}_{\text {win }}^{\mathrm{opt}} \mathbf{C}_{\mathbf{y}_{\mathcal{Q}_{\text {win }}}} \boldsymbol{\Delta}_{\text {win }}^{\mathrm{opt} H}\right)^{-1} \boldsymbol{\Delta}_{\text {win }}^{\mathrm{opt}} \mathbf{C}_{\mathbf{y}_{\mathcal{Q}_{\text {win }}} \mathbf{x}_{\text {win }}}
$$

and the detected symbols in one window are

$$
\tilde{\mathbf{x}}_{\text {win }}=\mathbf{W}_{\text {LRA-MMSE }}^{\mathrm{opt}^{H}} \boldsymbol{\Delta}_{\text {win }}^{\mathrm{opt}} \mathbf{y}_{\mathcal{Q}_{\text {win }}}
$$

\section{3}

\section{The Design of the Reduction Matrix}

In this section, design algorithms are presented for the reduction matrix $\Delta$ that operates on the oversampled signal and performs the dynamic oversampling by combining or choosing the samples according to the sum rate or the MSE criteria. In particular, algorithms are presented to solve the optimization problems (5-23) and (5-31), both of which can be generalized as

$$
\boldsymbol{\Delta}^{\mathrm{opt}}=\arg \max _{\boldsymbol{\Delta}} \operatorname{Tr}\left(\boldsymbol{\Delta} \mathbf{C}_{A} \boldsymbol{\Delta}^{H}\left(\boldsymbol{\Delta} \mathbf{C}_{B} \boldsymbol{\Delta}^{H}\right)^{-1}\right) .
$$


Assume that both $\mathbf{C}_{A}$ and $\mathbf{C}_{B}$ have the size of $M N N_{r} \times M N N_{r}$ and $\boldsymbol{\Delta}$ has the size of $M^{\prime} N N_{r} \times M N N_{r}$. The problem (5-35) is known as the ratio trace problem [81]. In the following, two algorithms will be illustrated to solve this problem, the generalized eigenvalue decomposition (GEVD) for obtaining a full complex reduction matrix and the submatrix-level feature selection (SL-FS) for getting a sparse binary matrix.

\subsection{1}

\section{Generalized Eigenvalue Decomposition}

From [81], the problem in (5-35) can be efficiently solved by the GEVD

$$
\mathbf{C}_{A} \delta_{c}=\lambda_{c} \mathbf{C}_{B} \delta_{c}
$$

where $\lambda_{c}$ is the cth largest generalized eigenvalue. The matrix $\boldsymbol{\Delta}^{\mathrm{opt}^{H}}$ is then constituted of the corresponding eigenvectors $\delta_{c}, c=1, \cdots, M^{\prime} N N_{r}$. A rigorous GEVD solution [82] is summarized in Alg. 3. In step $5, \boldsymbol{\Lambda}$ is a diagonal matrix containing all the eigenvalues and the corresponding eigenvectors are stored in $\boldsymbol{\Delta}$.

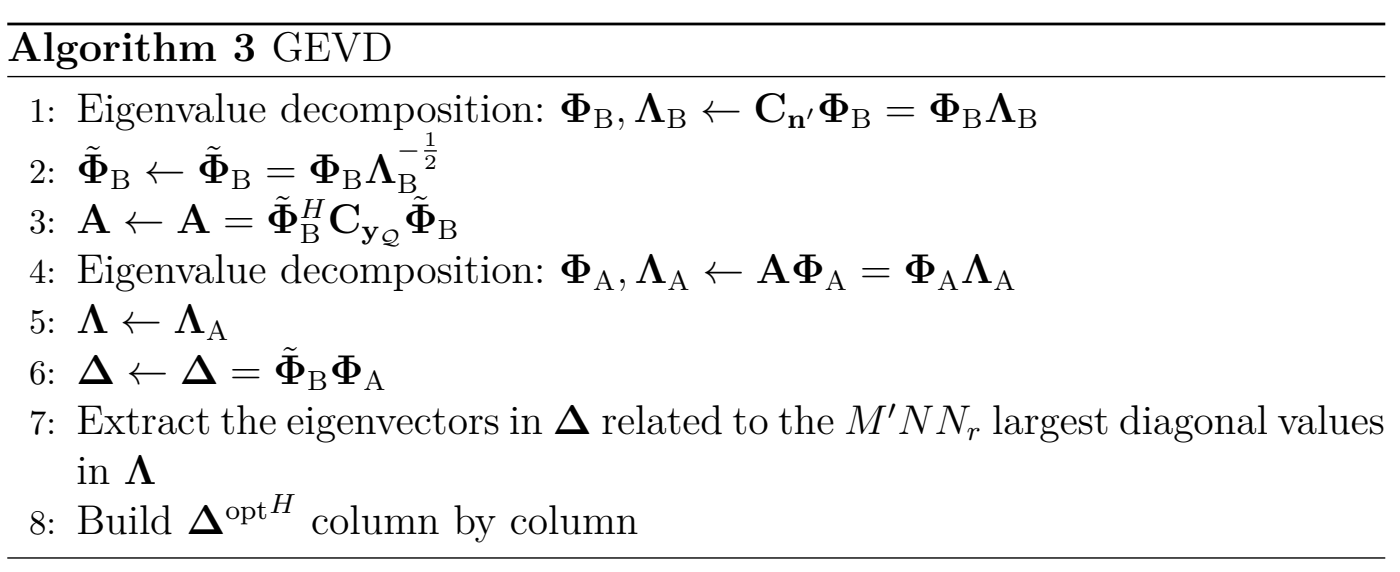

\section{3 .2}

\section{Submatrix-level Feature Selection}

The main drawback of the GEVD algorithm is the high computational cost of the eigenvalue decompositions and full matrix multiplications involved in (5-19) and (5-34). Considering this, a new approach is proposed, in which the reduction matrix is a sparse binary matrix only containing a single one in each row. The advantage of this binary matrix $\boldsymbol{\Delta}_{B}$ is that while multiplying with such matrix only a few data samples are selected. The samples associated with zeros in $\boldsymbol{\Delta}_{B}$ are discarded without the need for arithmetic operations, which can largely reduce the computational cost. The optimization problem (5-35) can be converted as 


$$
\boldsymbol{\Delta}_{B}^{\mathrm{opt}}=\underset{\boldsymbol{\Delta}_{B} \text { is sparse binary }}{\arg \max } \operatorname{Tr}\left(\boldsymbol{\Delta}_{B} \mathbf{C}_{A} \boldsymbol{\Delta}_{B}^{H}\left(\boldsymbol{\Delta}_{B} \mathbf{C}_{B} \boldsymbol{\Delta}_{B}^{H}\right)^{-1}\right) .
$$

However, (5-37) is not easy to be solved due to its combinatorial nature. The simplest but hardest way is to search all possible patterns of $\boldsymbol{\Delta}_{B}$ and select the best one. In large-scale MIMO systems, $\boldsymbol{\Delta}_{B}$ has a large dimensional size, which will consume much computational cost while doing the searching. To alleviate this, with the sparse characteristic of $\boldsymbol{\Delta}_{B}$ it is separated into several short dimensional matrices

$$
\boldsymbol{\Delta}_{B}=\operatorname{blkdiag}\left(\left[\boldsymbol{\Delta}_{B 1}, \boldsymbol{\Delta}_{B 2}, \cdots, \boldsymbol{\Delta}_{B K}\right]\right)
$$

and search the best pattern for each submatrix $\boldsymbol{\Delta}_{B k}(k=1, \cdots, K)$ with the size of $\frac{M^{\prime} N N_{r}}{K} \times \frac{M N N_{r}}{K}$. The choice of $K$ depends on the value of $M^{\prime} N N_{r}$, so that $\frac{M^{\prime} N N_{r}}{K}$ can not be very small nor large. Since a very short dimensional matrix $\boldsymbol{\Delta}_{B k}$ can not largely represent the original matrix $\boldsymbol{\Delta}_{B}$ and a very large dimensional matrix will make the searching complex, there should be a tradeoff $^{3}$. The optimization problem is then reduced to

$$
\boldsymbol{\Delta}_{B_{k}}^{\text {opt }}=\underset{\boldsymbol{\Delta}_{B_{k}}}{\arg \text { is marse binary }} \operatorname{Tr}\left(\boldsymbol{\Delta}_{B_{k}} \mathbf{C}_{A_{k}} \boldsymbol{\Delta}_{B_{k}}^{H}\left(\boldsymbol{\Delta}_{B_{k}} \mathbf{C}_{B_{k}} \boldsymbol{\Delta}_{B_{k}}^{H}\right)^{-1}\right),
$$

where $\mathbf{C}_{A_{k}}, \mathbf{C}_{B_{k}} \in \mathbb{C}^{\frac{M N N_{r}}{K} \times \frac{M N N_{r}}{K}}$ are block diagonal submatrices from $\mathbf{C}_{A}$ and $\mathbf{C}_{B}$, respectively.

In the following, two algorithms are proposed for searching the optimal pattern $\boldsymbol{\Delta}_{B_{k}}^{\mathrm{opt}}$, submatrix-level backward feature selection (SL-BFS) and submatrix-level restricted greedy search (SL-RGS).

\subsubsection{1}

\section{Backward Feature Selection}

Inspired by the feature selection algorithms used in machine learning and statistics [83], the idea of BFS is applied to search for $\boldsymbol{\Delta}_{B_{k}}^{\mathrm{opt}}$. The initialization is an identity matrix and remove the least significant row at each iteration. In the end, the rows contributing to the smallest trace in (5-39) are eliminated. The whole procedure is summarized in Alg. 4.

\subsubsection{2}

\section{Restricted Greedy Search}

The basic idea is that based on the initialized pattern $\Delta_{B_{k}}^{I}$ the best row patterns are sequentially searched from the first until the last row. While searching the $r$ th row pattern, the position of the one is shifted within a predefined small range and select the one contributing to the highest trace in

\footnotetext{
${ }^{3}$ For the system with a large receive antenna array $\left(N_{r} \geq 64\right), K$ can be chosen as $M^{\prime} N$.
} 


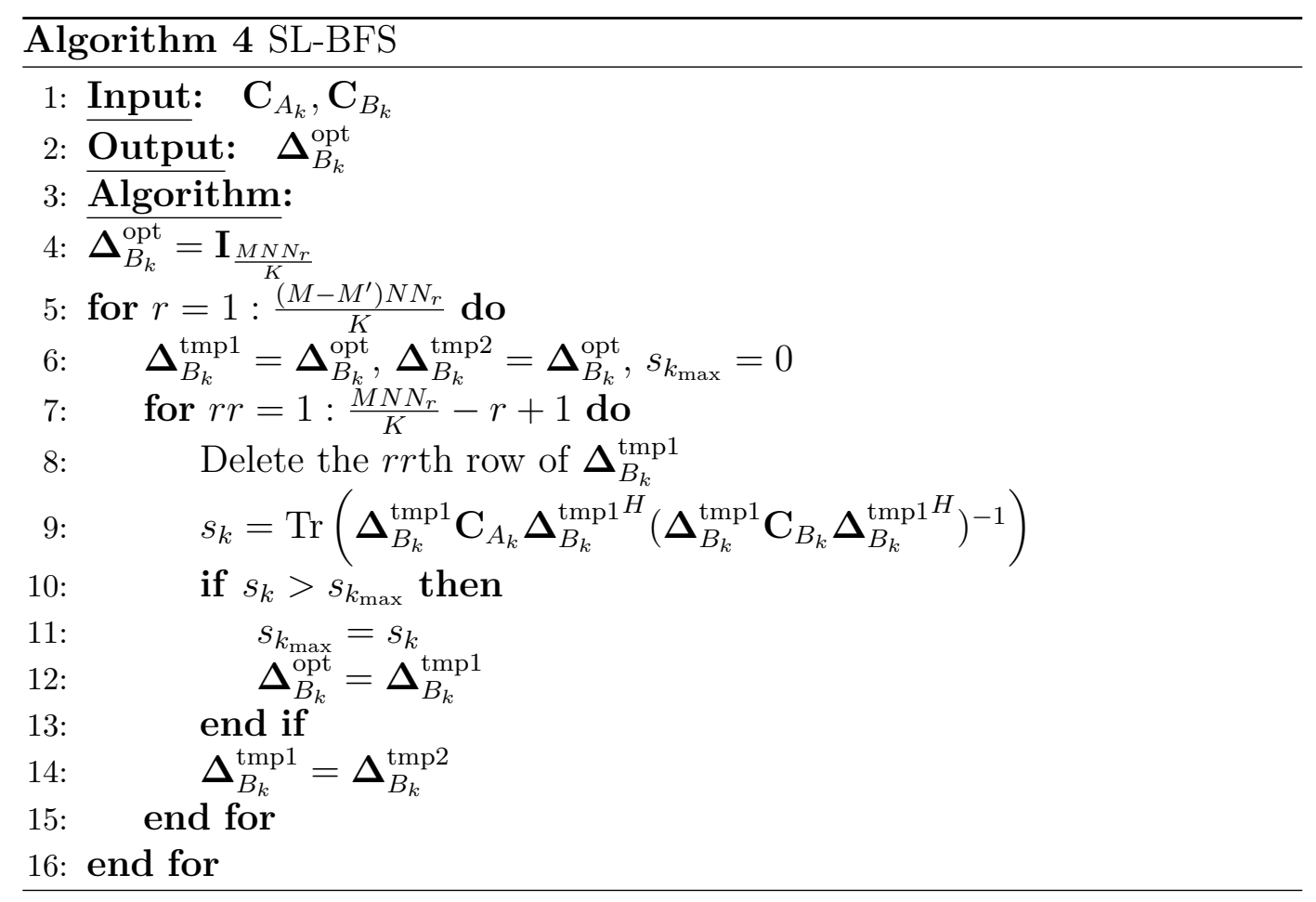

(5-39). While calculating the trace, the rest $\frac{M^{\prime} N N_{r}}{K}-1$ rows are fixed including the first $r-1$ optimized and the rest $\frac{M^{\prime} N N_{r}}{K}-r$ non-optimized rows. The proposed algorithm is described in Alg. 5.

The initialized pattern $\boldsymbol{\Delta}_{B_{k}}^{I}$ is the $k$ th block diagonal submatrix of $\boldsymbol{\Delta}_{B}^{I}$ (as (5-38)). The matrix $\boldsymbol{\Delta}_{B}^{I}$ is the initialized pattern for $\boldsymbol{\Delta}_{B}$ and selected as the pattern for uniform or quasi-uniform ${ }^{4}$ oversampling, i.e.,

$$
\boldsymbol{\Delta}_{B}^{I}=\left[\begin{array}{cccccccccccc}
0 & \cdots & 0 & 1 & 0 & 0 & 0 & 0 & \cdots & 0 & 0 & 0 \\
\vdots \vdots & r_{1} & \vdots & \vdots & \vdots & \vdots & \vdots & \vdots & \vdots & \vdots & \vdots & \vdots \\
0 & \cdots & 0 & 0 & 1 & 0 & 0 & 0 & \cdots & 0 & 0 & 0 \\
\vdots \vdots & & r_{r} & \vdots & \vdots & \vdots & \vdots & \vdots & \vdots & \vdots & \vdots & \vdots \\
0 & \cdots & 0 & 0 & 0 & 0 & 0 & 1 & \cdots & 0 & 0 & 0
\end{array}\right],
$$

where $r_{r}$ denotes the number of zeros before 1 in each row. In the following, examples are taken to illustrate how $\boldsymbol{\Delta}_{B}^{I}$ is chosen. For a system with $M=4$ (or $M=6$ ) and $M^{\prime}=2, \Delta_{B}^{I}$ can be easily found. Since the uniform sampling pattern in one Nyquist interval is $[1,0,1,0]$ (or $[1,0,0,1,0,0]$ for $M=6$ ), $r_{r}=M \frac{r-1}{M^{\prime}}$, where $r$ is the index of the row. However, for the system with $M=3$ (or $M=5$ ) and $M^{\prime}=2$, the quasi-uniform sampling pattern in one Nyquist interval has more alternatives, which can be either $[1,0,1]$ or $[1,1,0]$

${ }^{4}$ When $\frac{M}{M^{\prime}}$ is an integer, $\boldsymbol{\Delta}_{B}^{I}$ is the pattern for uniform oversampling, otherwise it is for quasi-uniform oversampling. 


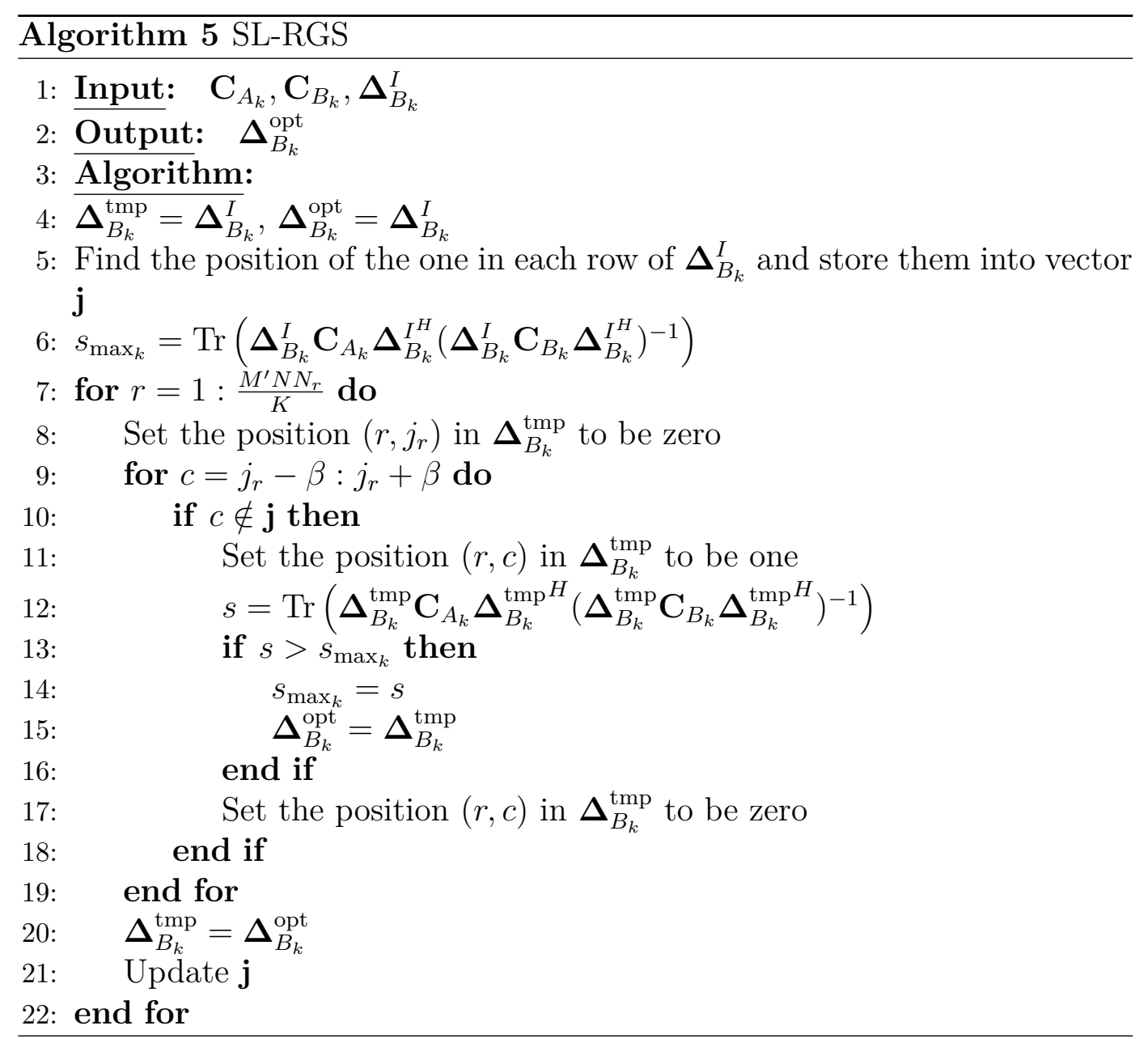

(either $[1,0,1,0,0]$ or $[1,0,0,1,0]$ for $M=5$ ).

$$
r_{r}= \begin{cases}M\left\lfloor\frac{r-1}{M^{\prime}}\right\rfloor, & \text { if } \bmod \left(r-1, M^{\prime}\right)=0 \\ M\left\lfloor\frac{r-1}{M^{\prime}}\right\rfloor+\alpha, & \text { otherwise, }\end{cases}
$$

where $\alpha$ denotes the position of the second one starting from position 0 .

In step $8, j_{r}$ denotes the position of the one at the $r$ th row and is stored in the vector $\mathbf{j} \in \mathbb{R}^{1 \times \frac{M^{\prime} N N_{r}}{K}}$. The parameter $\beta$ in step 9 is the pre-defined range number, which aims to reduce the cost of the search. The possible position of the one is only searched within the range of $2 \beta$ around $j_{r}$.

\section{4}

\section{Analysis of Proposed Scheme}

In this section, the following topics are illustrated: the convergence of the proposed SL-RGS algorithm, comparison of the computational complexities of different reduction algorithms and the power consumption of the proposed scheme at the receiver. The parameters used in this section are $M^{\prime}=2, N=4$, $N_{r}=64, \beta=5$ and $K=8$. 


\section{4 .1}

\section{Convergence of the SL-RGS Algorithm}

Let us consider a sparse dimension reduction submatrix $\boldsymbol{\Delta}_{B_{k}}$ defined in the previous section. The convergence of the proposed RGS algorithm to the unrestricted (or exhaustive searched) GS algorithm is examined under some reasonable assumptions. Note that the exhaustive search for $\boldsymbol{\Delta}_{B_{k}}^{\mathrm{opt}}$ results in $\left(\frac{\left(M-M^{\prime}\right) N N_{r}}{K}\right)^{\frac{M^{\prime} N N_{r}}{K}}$ independent calculations of trace (step 12 in Alg. 5), which is quite complex for practical use.

The proposed RGS algorithm greatly reduces the cost of the search (maximal $(2 \beta)^{\frac{M^{\prime} N N_{r}}{K}}$ independent calculations of trace) and under some conditions can approach the performance of unrestricted GS obtained $\boldsymbol{\Delta}_{B_{k}}^{\mathrm{opt}}$. Assume that the RGS examines a range of patterns characterized by

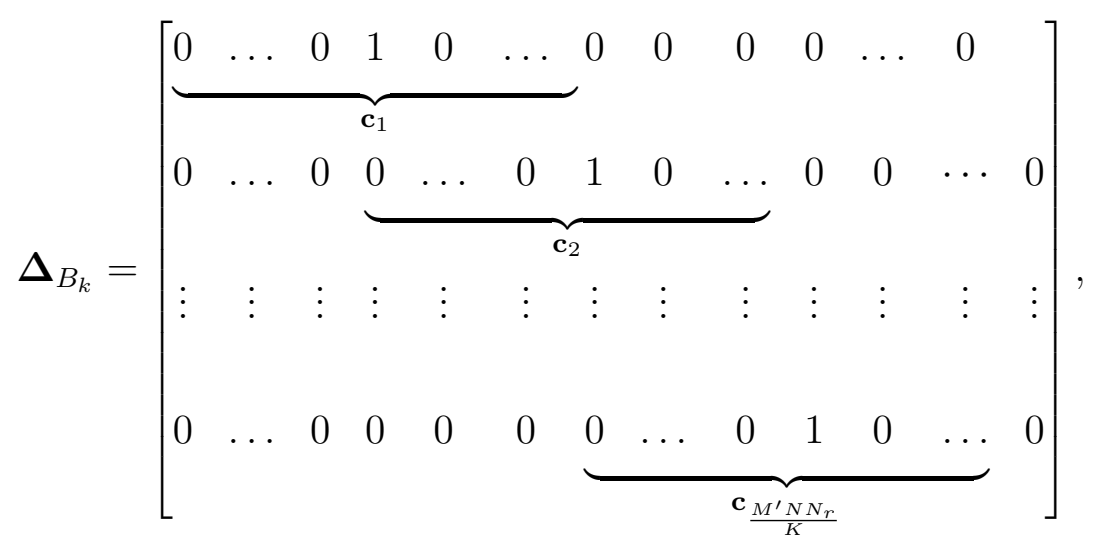

where $\mathbf{c}_{r}=\left[\begin{array}{lllll}0 & \ldots & 0 & 1 & 0\end{array} \ldots .0\right]\left(r=1,2, \ldots, \frac{M^{\prime} N N_{r}}{K}\right)$ is a row vector that has a length of $2 \beta+1$. The trace of the $r$ th optimized $\boldsymbol{\Delta}_{B_{k}}^{r}$ is calculated as

$$
s_{r}=\operatorname{Tr}\left(\boldsymbol{\Delta}_{B_{k}}^{r} \mathbf{C}_{A_{k}} \boldsymbol{\Delta}_{B_{k}}^{r}{ }^{H}\left(\boldsymbol{\Delta}_{B_{k}}^{r} \mathbf{C}_{B_{k}} \boldsymbol{\Delta}_{B_{k}}^{r}{ }^{H}\right)^{-1}\right) .
$$

In the following, an example is taken to illustrate how

$$
s_{r} \rightarrow s_{\text {optimum }}
$$

where $s_{\text {optimum }}$ is the trace obtained by the unrestricted GS with the form as

$$
\boldsymbol{\Delta}_{B_{k}}^{\text {optmum }}=\left[\begin{array}{ccccccccccccc}
0 & 1 & 0 & 0 & 0 & 0 & 0 & 0 & 0 & 0 & 0 & \ldots & 0 \\
0 & 0 & 0 & 0 & 0 & 0 & 0 & 0 & 1 & 0 & 0 & \ldots & 0 \\
0 & \ldots & 0 & 0 & 1 & 0 & 0 & 0 & 0 & 0 & 0 & 0 & 0
\end{array}\right] .
$$

In the RGS algorithm, the initialized pattern $\boldsymbol{\Delta}_{B_{k}}^{I}$ is 


$$
\boldsymbol{\Delta}_{B_{k}}^{I}=\left[\begin{array}{ccccccccccccc}
1 & 0 & 0 & 0 & 0 & 0 & 0 & 0 & 0 & 0 & 0 & \ldots & 0 \\
0 & 0 & 1 & 0 & 0 & 0 & 0 & 0 & 0 & 0 & 0 & \ldots & 0 \\
0 & \ldots & 0 & 0 & 0 & 0 & 0 & 0 & 0 & 0 & 0 & 0 & 1
\end{array}\right]
$$

After the first iteration, we obtain

$$
\boldsymbol{\Delta}_{B_{k}}^{1}=\left[\begin{array}{ccccccccccccc}
0 & 0 & 0 & 1 & 0 & 0 & 0 & 0 & 0 & 0 & 0 & \ldots & 0 \\
0 & 0 & 1 & 0 & 0 & 0 & 0 & 0 & 0 & 0 & 0 & \ldots & 0 \\
0 & \ldots & 0 & 0 & 0 & 0 & 0 & 0 & 0 & 0 & 0 & 0 & 1
\end{array}\right]
$$

After the $\frac{M^{\prime} N N_{r}}{K}$ th iteration, we have

$$
\boldsymbol{\Delta}_{B_{k}{ }_{M^{\prime} N N_{r}}^{K}}=\left[\begin{array}{lllllllllllll}
0 & 0 & 0 & 1 & 0 & 0 & 0 & 0 & 0 & 0 & 0 & \ldots & 0 \\
0 & 0 & 0 & 0 & 0 & 1 & 0 & 0 & 0 & 0 & 0 & \ldots & 0 \\
0 & \ldots & 0 & 0 & 0 & 0 & 0 & 0 & 0 & 1 & 0 & 0 & 0
\end{array}\right] .
$$

Fig. 5.2 shows the corresponding convergence performance, where $r=$ $1,2, \cdots, 64$. It can be seen that although $\Delta_{B_{k}{ }^{K}}^{\frac{M^{\prime} N N_{r}}{K}}$ and $\Delta_{B_{k}}^{\text {optmum }}$ do not have similar patterns the difference $\left(s_{\text {optimum }}-s_{r}\right)$ still decreases as the number of iterations increases until it approaches zero. This indicates that the RGS might lead to a close to optimal result in terms of $s_{r}$, i.e. $s_{r} \rightarrow s_{\text {optimum }}$ provided that the range $2 \beta$ is sufficiently large.

This discussion aims to give insight on how the proposed RGS can obtain satisfactory results provided the range $2 \beta$ is chosen sufficiently large.

\section{4 .2}

\section{Computational Complexity}

\subsubsection{1}

\section{GEVD vs SL-FS}

In the GEVD algorithm, the operations that contribute the most to the computational complexity lie in the eigenvalue decomposition $\left(\mathcal{O}\left(\left(M N N_{r}\right)^{3}\right)\right.$ in [85]) and matrix multiplications. While in the SL-FS algorithms, the opera- 


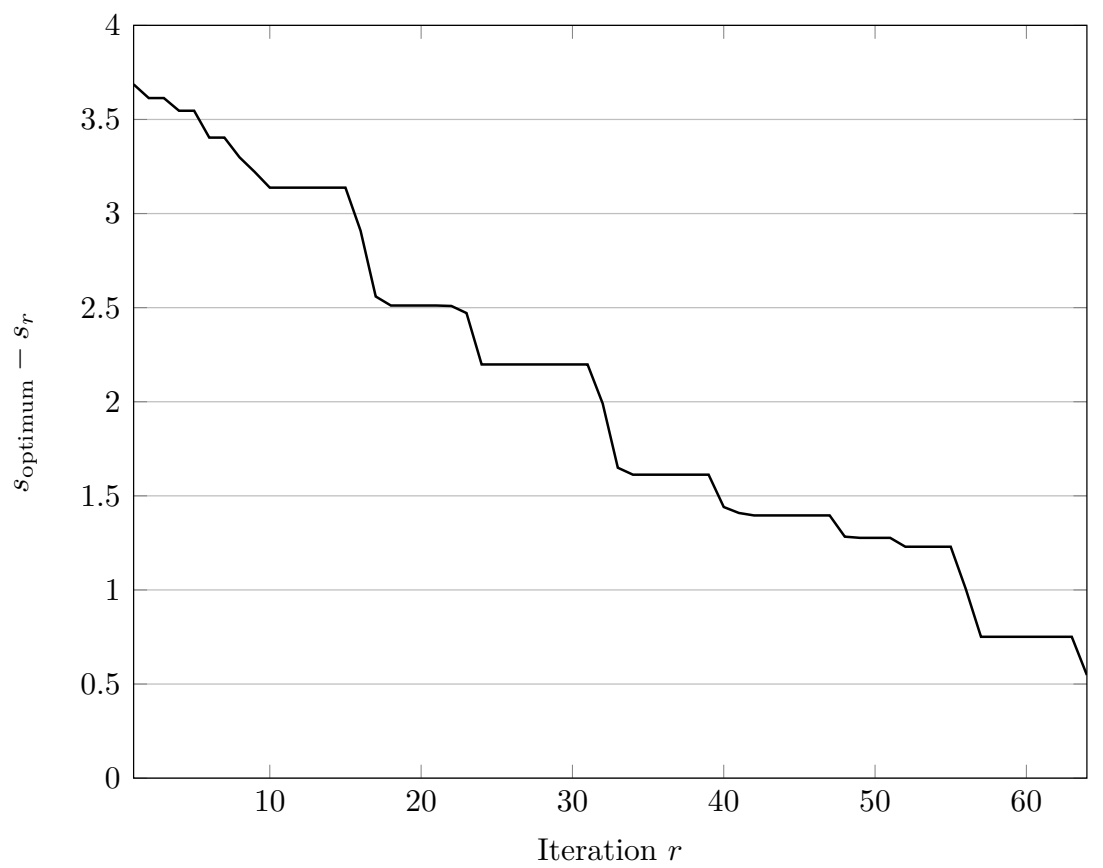

Figure 5.2: Convergence of the proposed SL-RGS to the unrestricted GS algorithm.

tion of trace in each iteration consumes most of the calculations. The computational complexities of the illustrated design algorithms are shown in Table 5.1. The solid lines in Fig. 5.3 represent the computational complexities of three

Table 5.1: Computational complexities of the illustrated matrix design algorithms

\begin{tabular}{|c|c|c|}
\hline GEVD & SL-BFS & SL-RGS \\
\hline $\mathcal{O}\left(6\left(M N N_{r}\right)^{3}\right)$ & $\mathcal{O}\left(\sum_{n=1}^{\frac{\left(M-M^{\prime}\right) N N_{r}}{K}} 2 K\left(\frac{M N N_{r}}{K}-n+1\right)\left(\left(\frac{M N N_{r}}{K}-n\right)^{3}+\right.\right.$ & $\mathcal{O}\left(4 M^{\prime} N N_{r} \beta\left(\left(\frac{M^{\prime} N N_{r}}{K}\right)^{3}\right.\right.$ \\
& $\left.\left.\left(\frac{M N N_{r}}{K}-n\right)^{2} \frac{M N N_{r}}{K}+\left(\frac{M N N_{r}}{K}-n\right)\left(\frac{M N N_{r}}{K}\right)^{2}\right)\right)$ & $\left.\left.+\left(\frac{M^{\prime} N N_{r}}{K}\right)^{2} \frac{M N N_{r}}{K}+\frac{M^{\prime} N N_{r}}{K}\left(\frac{M N N_{r}}{K}\right)^{2}\right)\right)$ \\
\hline
\end{tabular}

matrix design algorithms. It can be seen that the SL-RGS algorithm consumes roughly the same complexity as the GEVD and the SL-BFS algorithm has the highest complexity.

Noticing that for obtaining the whole $\boldsymbol{\Delta}_{B}^{\mathrm{opt}}$, the SL-FS algorithm needs to be repeated for $K$ times in order to obtain all optimal submatrices $\boldsymbol{\Delta}_{B_{k}}^{\mathrm{opt}}$ $(k=1, \cdots, K)$. To further reduce the complexity of the SL-FS algorithm, a simplified version is proposed, in which it is assumed that all optimal submatrices $\boldsymbol{\Delta}_{B_{k}}^{\mathrm{opt}}(k=1, \cdots, K)$ share the same pattern, so that only one submatrix $\boldsymbol{\Delta}_{B_{k}}$ will be optimized by the SL-FS algorithm. The choice of which $\boldsymbol{\Delta}_{B_{k}}$ is optimized is influenced by the minimal trace calculated by its initial pattern, which is shown from step 1 to 4 in Alg. 6. The complexity of the simplified SL-BFS and SL-RGS are shown as the dashed lines in Fig. 5.3. Compared to the standard algorithm, the simplified SL-FS can provide savings of $87.5 \%$ in the computational cost. 


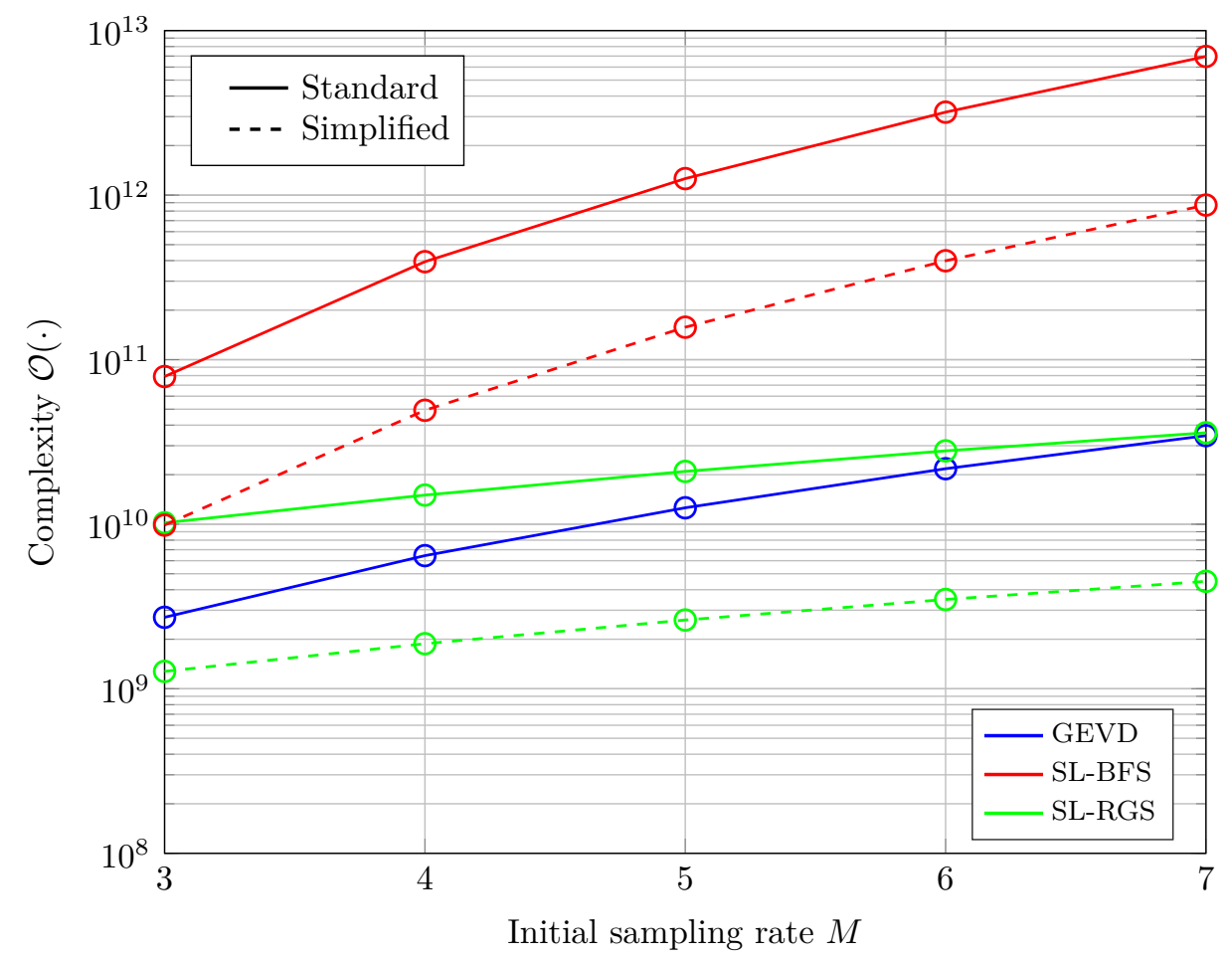

Figure 5.3: Comparison of computational complexities among illustrated matrix design algorithms.

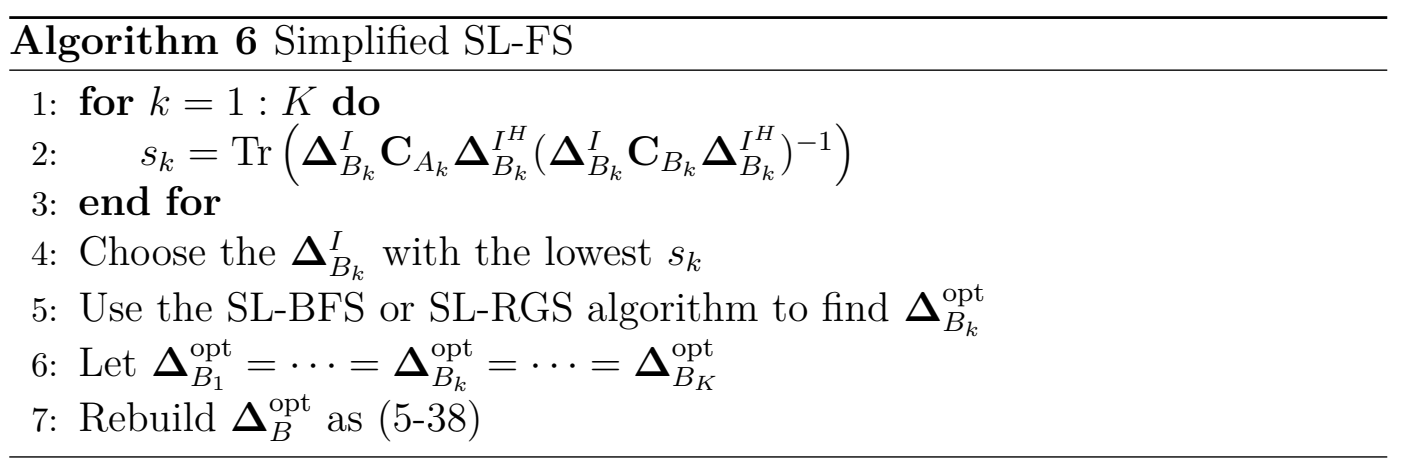

\subsubsection{2}

\section{Uniform vs Dynamic Oversampling}

In the uniform-oversampled system, all received samples are used for the signal processing, which means that there is no need for the pattern design and dimension reduction. The corresponding sliding window based LRA-MMSE detector is a modified version of (5-33), which is

$$
\mathbf{W}_{\text {LRA-MMSE }}^{\text {uni }}=\mathbf{C}_{\mathbf{y}_{\mathcal{Q}_{\text {win }}}^{-1}}^{-\mathbf{C}_{\mathbf{y}_{\mathcal{Q}_{\text {win }}} \mathbf{x}_{\text {win }}}} \text {. }
$$

Table 5.2 shows the computational complexities of different oversampling techniques for obtaining the detected symbols in each window. Considering the whole transmission block including the operation of pattern design, which is obtained only once at the beginning of each transmission, and dimension reduction, which is applied in each window, in dynamic oversampled systems, 
Table 5.2: Computational complexity by using different oversampling techniques in MSE based system

\begin{tabular}{|c|c|c|}
\hline $\begin{array}{c}\text { Uniform } \\
\text { oversampling }\end{array}$ & GEVD based & $\begin{array}{c}\text { SL-FS based } \\
\text { dynamic oversampling }\end{array}$ \\
\hline $\begin{array}{c}\mathcal{O}\left((M N N r)^{3}+(M N N r)^{2} l N t\right. \\
+(M N N r) l N t)\end{array}$ & $\mathcal{O}\left(\left(M^{\prime} N N_{r}\right)^{3}+\left(M^{\prime} N N_{r}\right)^{2}\left(l N t+M N N_{r}\right)\right.$ & $\mathcal{O}\left(\left(M^{\prime} N N r\right)^{3}+\left(M N^{\prime} N N r\right)^{2} l N t\right.$ \\
$\left.+\left(M^{\prime} N N_{r}\right)\left(\left(M N N_{r}\right)^{2}+M N N_{r} l N_{t}+M N N_{r}+l N_{t}\right)\right)$ & $\left.+\left(M^{\prime} N N r\right) l N t\right)$ \\
\hline
\end{tabular}

the total complexity comparisons between uniform and dynamic oversampling techniques are shown in Fig. 5.4. It can be seen that both the standard and simplified SL-RGS based dynamic oversampling technique consume the lowest computational cost among the listed oversampling techniques. Compared to the standard algorithm, the simplified SL-BFS and SL-RGS has saved up to $87.33 \%$ and $63.19 \%$ in the computational cost, respectively. When comparing with the uniform oversampling technique, the GEVD and the simplified SLRGS based dynamic oversampling technique can save up to $54.99 \%$ and $96.84 \%$ in the computational cost, respectively.

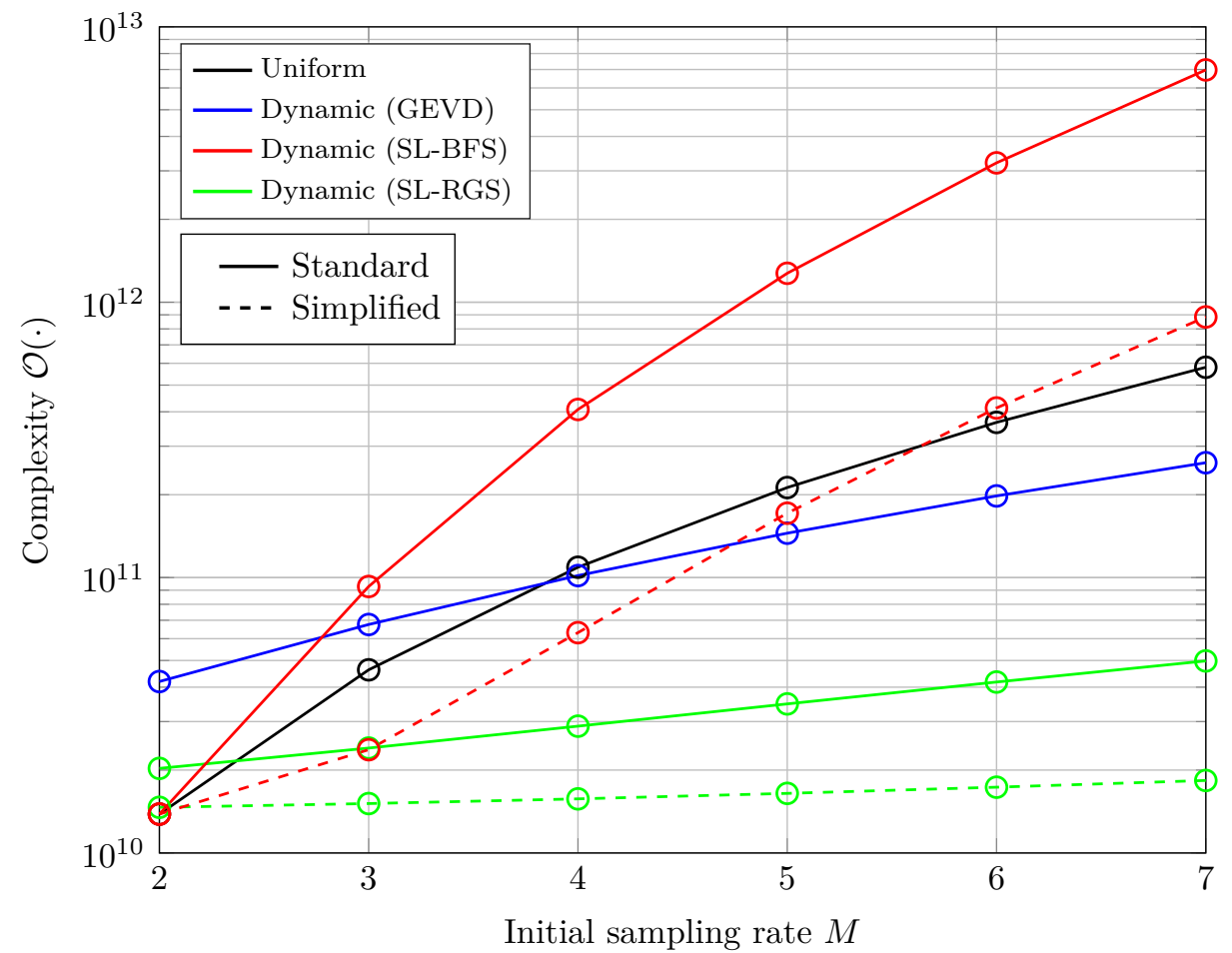

Figure 5.4: Computational complexity comparisons between uniform $\left(M=M^{\prime}\right)$ and dynamic oversampling techniques $\left(M^{\prime}=2\right)$.

\section{5}

\section{Numerical Results}

In this section, an uplink large-scale MIMO system with $N_{r}=64$ and $N_{t}=4$ is considered. The $m(t)$ and $p(t)$ are normalized RRC filters with a roll-off factor of 0.8 and the time delay $n_{n_{t}}^{d}$ of each terminal is uniformly 
distributed between $-T$ and $T$. The channel is assumed to experience block fading. The simulation results presented here are obtained by averaging over 500 independent realizations of the channel matrix $\mathbf{H}^{\prime}$, noise and symbol vectors. The SNR is defined as $10 \log \left(\frac{1}{\sigma_{n}^{2}}\right)$. In the SL-BFS and SL-RGS based dynamic oversampling technique, $\beta=5$ and $K=8$ are used.

\subsection{1}

\section{Sum Rate based System}

In this system, each transmission block contains 4 symbols and Gaussian signaling is considered. Fig. 5.5 shows the sum rate performance of the systems with uniform and GEVD based dynamic oversampling technique, where the latter has much better performance than the former. Fig. 5.6 compares the performance of SL-RGS based dynamic oversampling technique with standard and simplified version. As depicted in the figure, although there is performance degradation of the simplified algorithm the performance gaps between these two versions are still small, especially after $M=4$. Taking the complexity analysis (subsection 5.4.2) into account, it can be concluded that the proposed simplified SL-FS algorithm requires much lower computational cost to approach the performance of the standard SL-FS. Fig. 5.7 compares the performance of the simplified SL-FS algorithms, where both SL-BFS and SL-RGS achieve similar sum rates ${ }^{5}$.

\section{5 .2}

\section{MSE based System}

In this subsection, the proposed oversampling technique is examined in terms of the normalized MSE and SER. The modulation scheme is quadrature phase-shift keying (QPSK). Each transmission block contains 100 symbols. The window length $l$ is chosen as 4 Nyquist-sampled symbols.

Fig. 5.8 and Fig. 5.9 show the performance of normalized MSE and SER as a function of SNR in GEVD based dynamic oversampled systems, respectively. Similar to the Fig. 5.5, the dynamic oversampled system outperforms the uniform-oversampled systems. Combining with the complexity analysis in Fig. 5.4, it can be seen that the dynamic oversampling technique with $M=3$ performs much better than the uniform oversampling technique at the price of approximate similar computational costs.

Furthermore, Fig. 5.10 and Fig. 5.11 show the normalized MSE and SER performance of SL-RGS based dynamic oversampling technique with standard

\footnotetext{
${ }^{5}$ The performance of the standard SL-BFS algorithm is not shown due to the very high computational cost shown in subsection 5.4.2.
} 


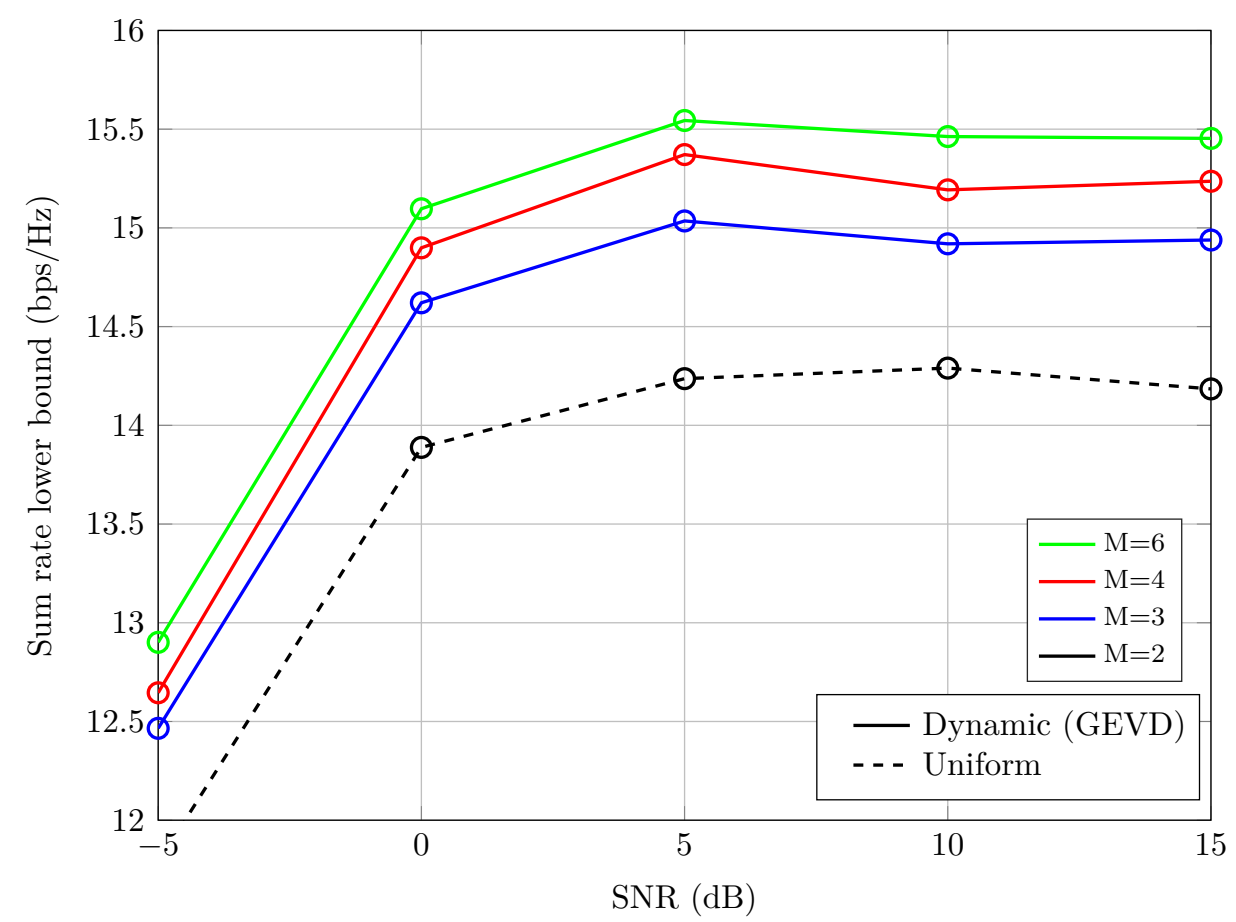

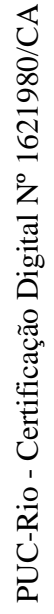

Figure 5.5: Comparison between uniform and GEVD based dynamic oversampling technique with $M^{\prime}=2$.

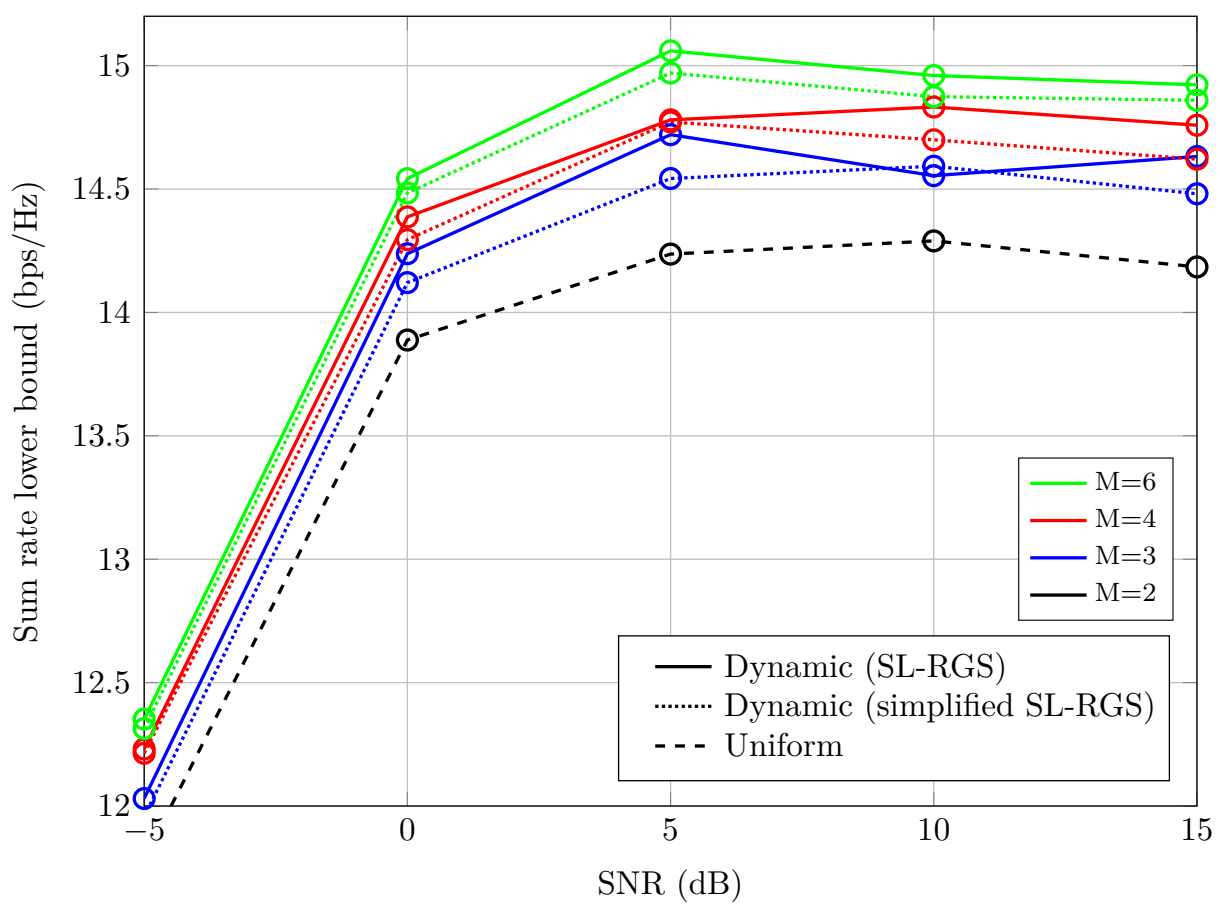

Figure 5.6: Comparison between uniform and SL-RGS based dynamic oversampling technique with $M^{\prime}=2$. 


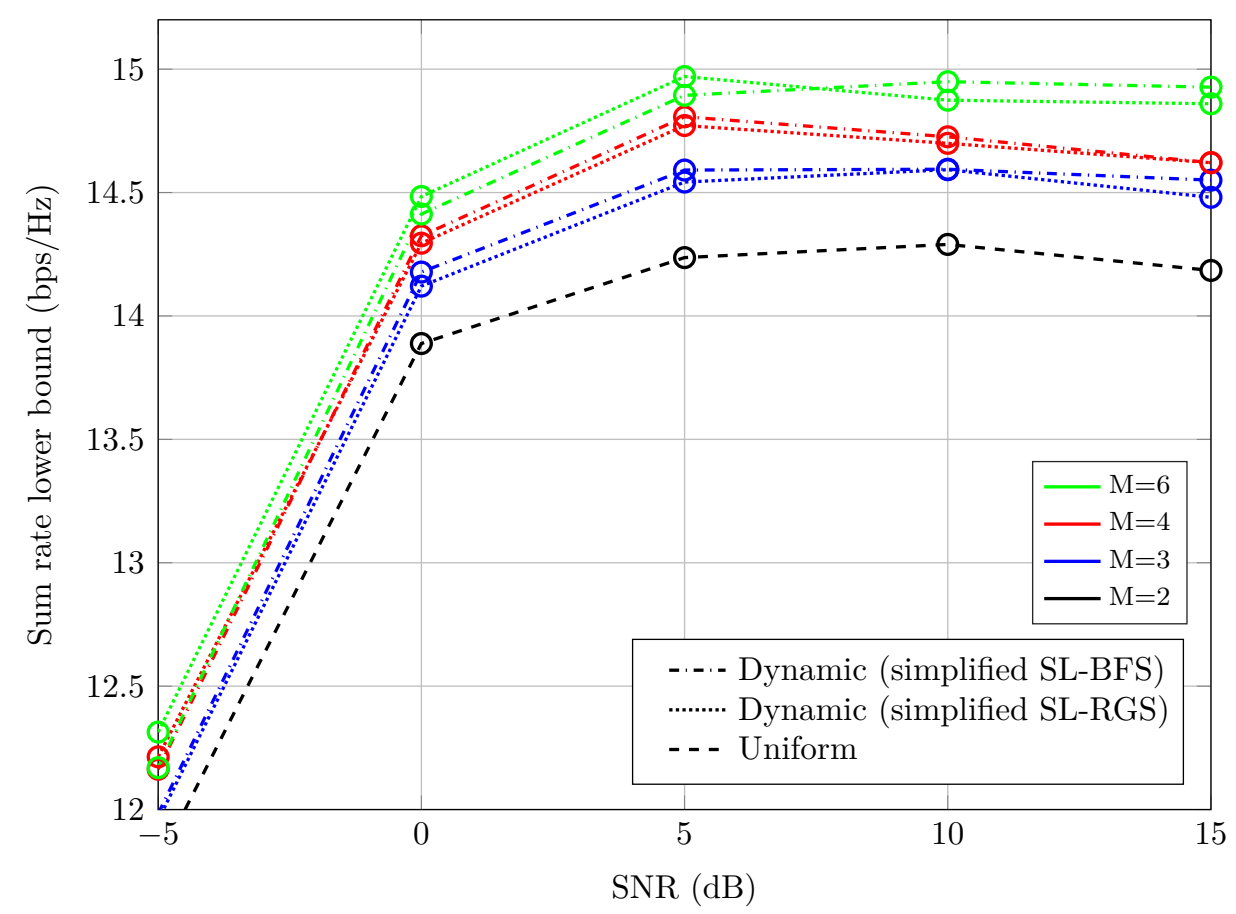

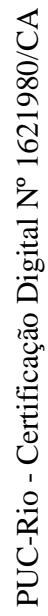

Figure 5.7: Comparison between simplified SL-BFS and simplified SL-RGS based dynamic oversampling technique with $M^{\prime}=2$.

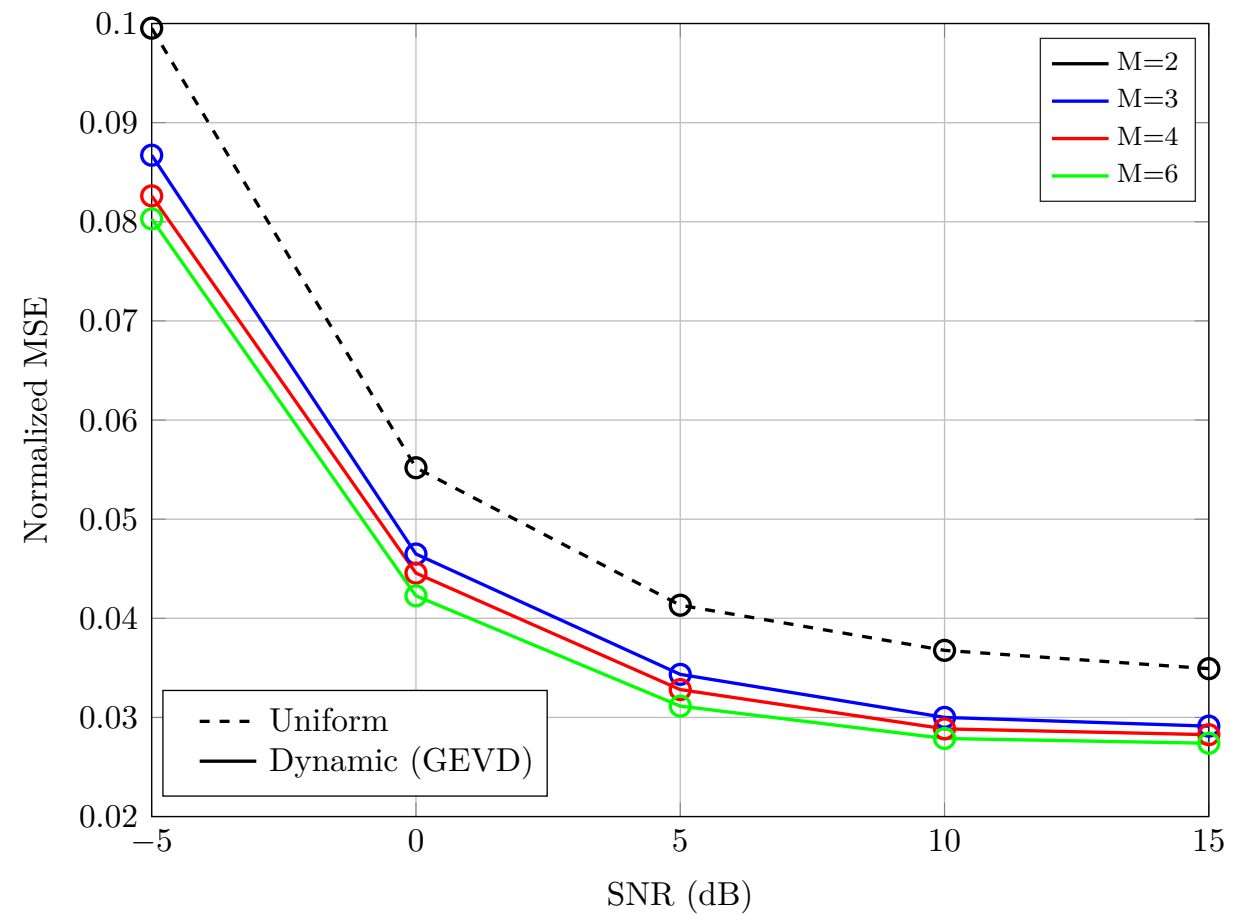

Figure 5.8: Comparison between uniform and GEVD based dynamic oversampling technique with $M^{\prime}=2$. 


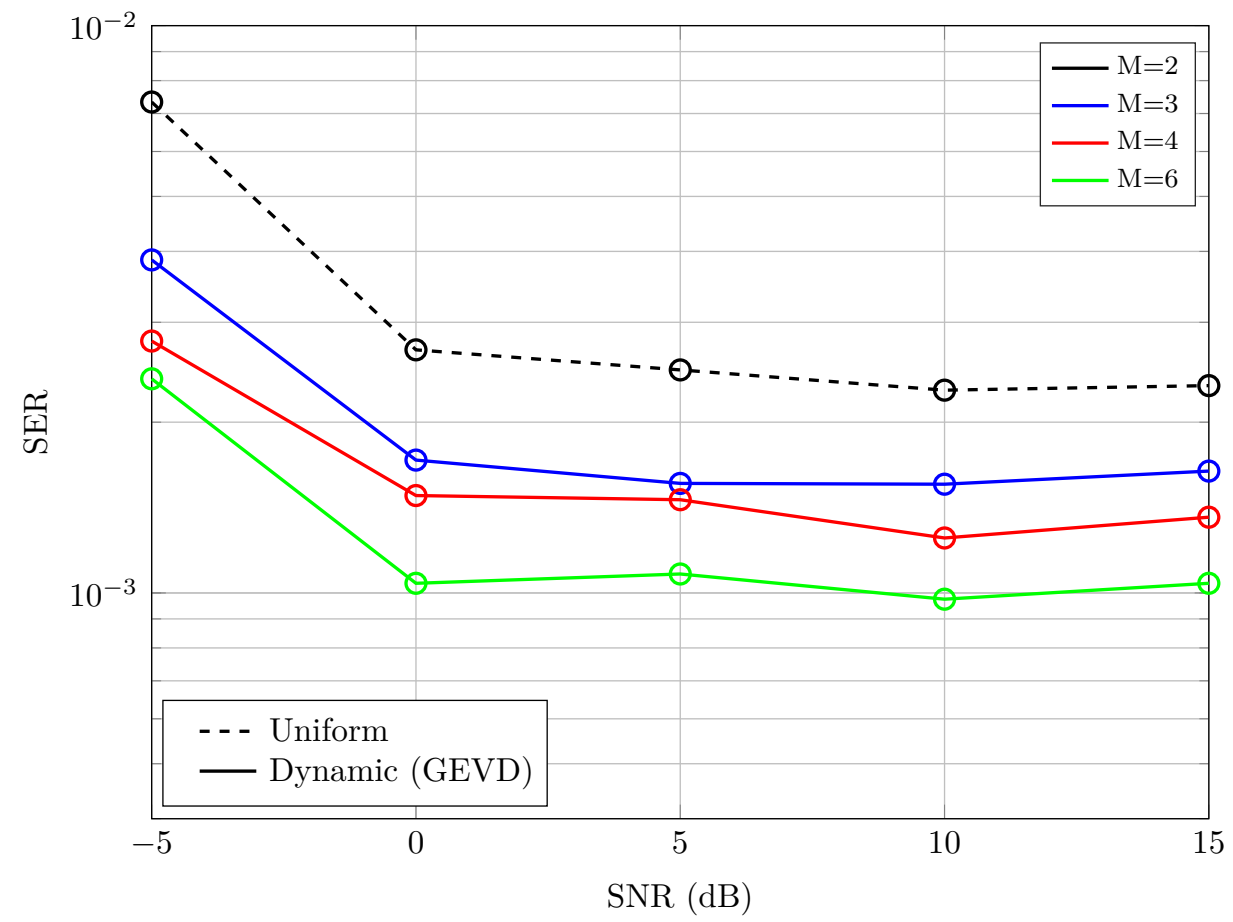

Figure 5.9: Comparison between uniform and GEVD based dynamic oversampling technique with $M^{\prime}=2$.

and simplified version. It can be seen that the performance of two proposed versions have little difference. Combining with the complexity analysis in Fig. 5.4, the simplified SL-RGS based dynamic oversampling technique is more suitable for the practical use since the computational cost is much lower. Moreover, it can also be seen that the dynamic oversampling technique obviously outperforms the uniform oversampling technique. with considering the complexity analysis in Fig. 5.4, it can be concluded that the proposed simplified SL-RGS based dynamic oversampling technique manages to achieve better normalized MSE and SER performance than the uniform oversampling technique with almost the same computational cost. For example, when $M=6$ the complexity of detecting one block of symbols in the simplified SL-RGS based dynamic oversampled system is almost similar with that in the uniformoversampled system but the performance has improved a lot. This reveals the performance advantages of the proposed dynamic oversampling technique. Similar to Fig. 5.7, the SER performance between the simplified SL-BFS and the simplified SL-RGS algorithm is also compared in Fig. 5.12, where both have similar performance. 


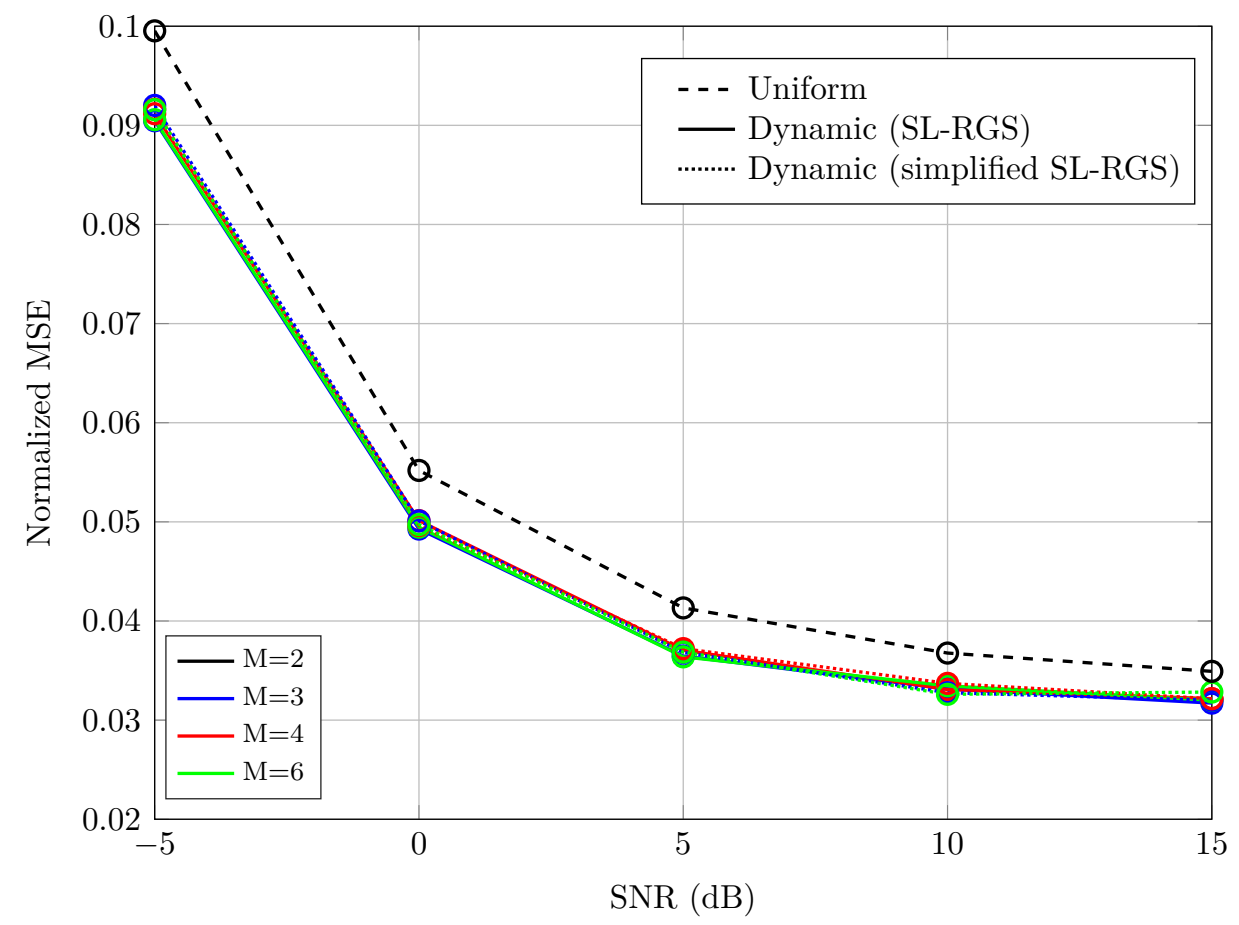

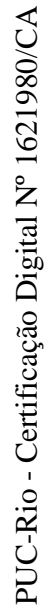

Figure 5.10: Comparison between uniform and SL-RGS based dynamic oversampling technique with $M^{\prime}=2$.

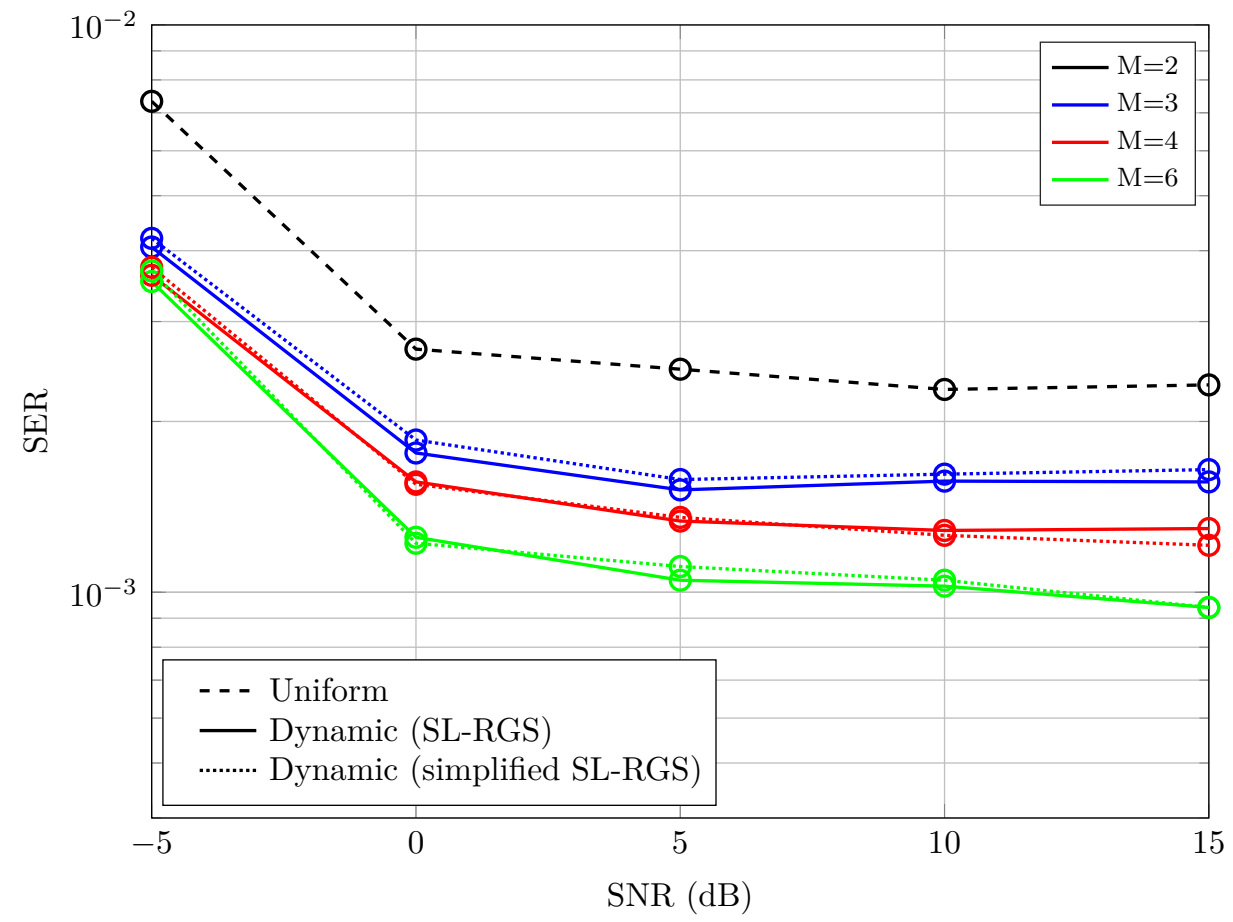

Figure 5.11: Comparison between uniform and SL-RGS based dynamic oversampling technique with $M^{\prime}=2$. 


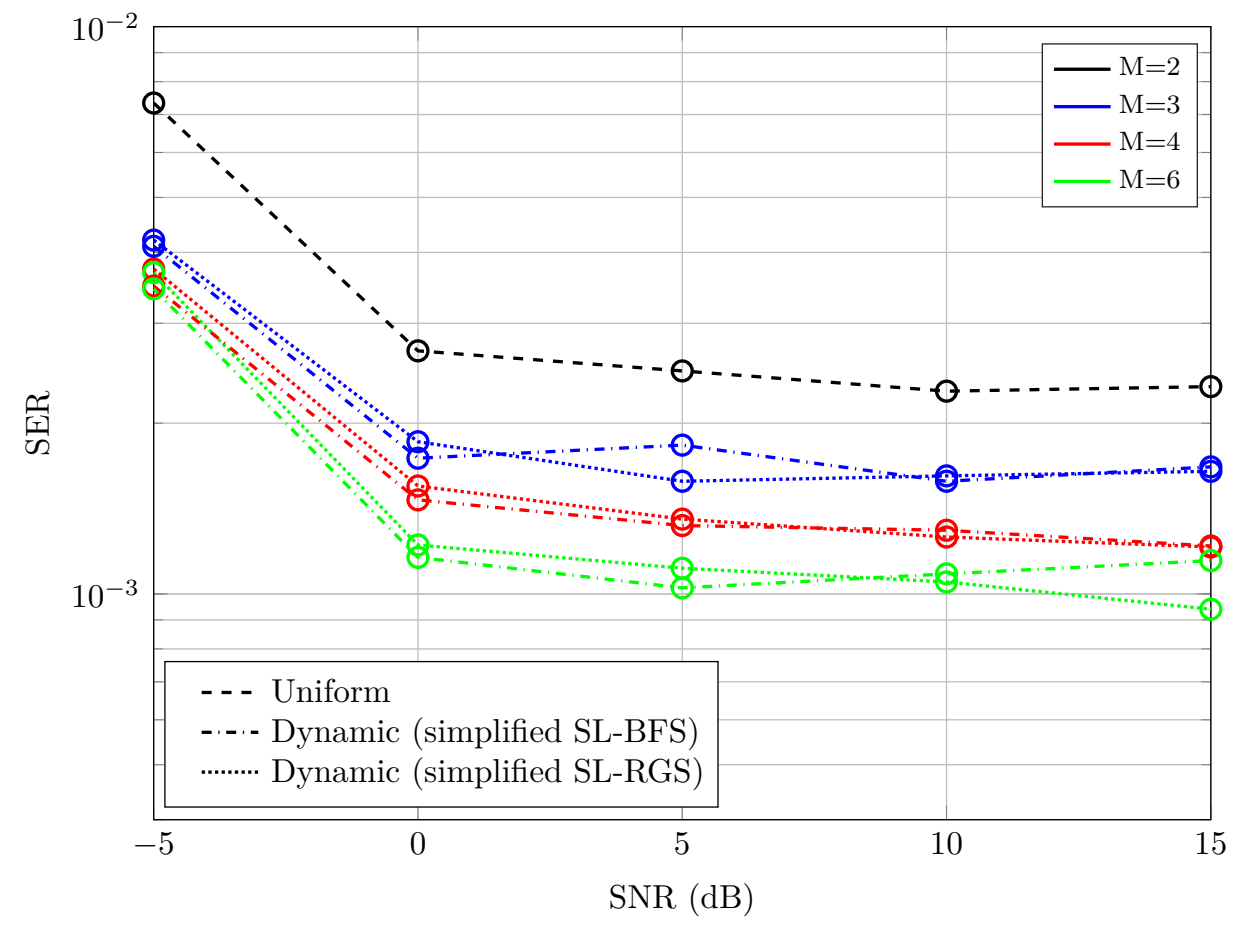

Figure 5.12: Comparison between simplified SL-BFS and simplified SL-RGS based dynamic oversampling technique with $M^{\prime}=2$.

\section{6}

\section{Summary}

This chapter has proposed dynamic oversampling techniques for largescale MU-MIMO systems with 1-bit quantization at the receiver. The designs based on the sum rate and MSE of detected symbols criteria are developed. Two algorithms have been devised for obtaining the dimension reduction matrix, the GEVD for the complex full matrix $\boldsymbol{\Delta}$ and the SL-FS for the sparse binary matrix $\boldsymbol{\Delta}_{B}$. Furthermore, the proposed scheme is analyzed based on the convergence, computational complexity and power consumption at the receiver. Simulation results have shown that the proposed dynamic oversampling techniques outperform the conventional uniform oversampling technique in terms of the computational cost, achievable sum rate and SER performance. 


\section{6}

\section{Conclusions and Future Works}

This thesis has developed novel signal processing techniques for largescale MU-MIMO systems with 1-bit quantization at the receiver that can provide energy-efficient algorithmic solutions for future wireless communication systems.

Chapter 2 has described the technical background on MU-MIMO systems, channel estimation, multiuser detection, precoding technique and lowresolution signal processing related to this thesis. In the section of MU-MIMO, the basic system model is firstly illustrated. Based on the model, sum rate capacity is given for a reliable transmission. While estimating the channel information, LS and LMMSE estimators are presented. The section of multiuser detection has discussed some optimum and sub-optimum detectors. In the section of low-resolution signal processing, the function of ADCs is explained in a mathematical way and some statistical properties of 1-bit ADCs are also presented. The use of oversampling aims at increasing the SQNR to mitigate the quantization error caused by the ADCs. The ADCs' power consumption among different resolutions is also compared.

Chapter 3 has shown the system design for MU-MIMO systems with coarsely quantized signals. Different from prior works on channel estimation in 1-bit ADCs system, low-resolution-aware LMS and RLS adaptive channel estimators are proposed to reduce the computational complexity while obtaining high accuracy. While making the signal detection, an IDD scheme together with an adaptive channel decoder using a quantizer is described. The simulation results have shown a great performance gain after several iterations in terms of BER.

To further improve the system performance, chapter 4 has presented the system design for 1-bit MU-MIMO systems with uniform oversampling technique. Based on different estimation criteria novel linear channel estimators are proposed for the systems sampling at oversampling rate. From the simulation results, it can be seen that there is about $2 \mathrm{~dB}$ SNR advantage with the non-oversampled system in terms of MSE. The LMS adaptive technique is also investigated in such systems. Numerical results have shown that the computational cost can be largely reduced but the MSE performance is almost the 
same as that of the LRA-LMMSE channel estimator, which is very important for systems with low computational complexity and simple hardware implementation. In addition, Bayesian and general CRBs on the MSE are derived, which give theoretical limits on the performance of the channel estimators. Furthermore, an adaptive technique to estimate the auto-correlation matrix of the channel vector is proposed, which is important for practical use. Novel signal detection algorithms are also presented for 1-bit systems sampling at uniform oversampling rate. To reduce the computational complexity caused by extra oversampled samples sliding-window based low-resolution-aware ZF and MMSE detectors have been developed. Simulation results have shown that there is performance gain in SER compared to the standard non-oversampled system.

Chapter 5 has proposed dynamic oversampling techniques for asynchronous large-scale MU-MIMO systems with 1-bit quantization at the receiver. Two system designs are developed based on the sum rate and the MSE of detected symbols criteria. Two algorithms have been devised for obtaining the dimension reduction matrix, the GEVD for the complex full matrix $\Delta$ and the SL-FS for the sparse binary matrix $\boldsymbol{\Delta}_{B}$. Furthermore, the proposed scheme is analyzed based on the convergence, computational complexity and power consumption at the receiver. Simulation results have shown that the proposed dynamic oversampling techniques outperform the conventional uniform oversampling technique in terms of the computational cost, achievable sum rate and SER performance.

Some suggestions for possible future works are:

- Pilot optimization: until now all the works about channel estimation in 1-bit ADCs systems consider the orthogonal pilots. However, due to the large quantization loss this configuration may not be optimal to the system. It would be promising to find out the optimal pilot patterns.

- B-bit ADCs: it is known that the 1-bit ADCs can produce a lot of quantization error, which makes the signal processing even more difficult. It would be interesting to investigate the use of b-bit $(b=2,3)$ ADCs on large-scale MU-MIMO systems including the development of signal detection, channel estimation, oversampling and precoding techniques. 


\section{Bibliography}

[1] DAHLMAN, PARKVALL, S.; BEMING. 3G Evolution: HSPA and LTE for Mobile Broadband. Academic Press, Oct. 2007.

[2] LARSSON, E. G.; EDFORS, O.; TUFVESSON, F. ; MARZETTA, T. L.. Massive MIMO for next generation wireless systems. IEEE Communications Magazine, 52(2):186-195, Feb. 2014.

[3] RUSEK, F.; PERSSON, D.; LAU, B. K.; LARSSON, E. G.; MARZETTA, T. L.; EDFORS, O. ; TUFVESSON, F.. Scaling up MIMO: Opportunities and challenges with very large arrays. IEEE Signal Processing Magazine, 30(1):40-60, Jan. 2013.

[4] ANDREWS, J. G.; BUZZI, S.; CHOI, W.; HANLY, S. V.; LOZANO, A.; SOONG, A. C. K. ; ZHANG, J. C.. What will 5G be? IEEE Journal on Selected Areas in Communications, 32(6):1065-1082, Jun. 2014.

[5] WALDEN, R. H.. Analog-to-digital converter survey and analysis. IEEE Journal on Selected Areas in Communications, 17(4):539-550, Apr. 1999.

[6] JACOBSSON, S.; DURISI, G.; COLDREY, M.; GUSTAVSSON, U. ; STUDER, C.. Throughput analysis of massive MIMO uplink with low-resolution ADCs. IEEE Transactions on Wireless Communications, 16(6):4038-4051, Jun. 2017.

[7] MO, J.; HEATH, R. W.. Capacity analysis of one-bit quantized MIMO systems with transmitter channel state information. IEEE Transactions on Signal Processing, 63(20):5498-5512, Oct. 2015.

[8] SAXENA, A. K.; FIJALKOW, I. ; SWINDLEHURST, A. L.. Analysis of one-bit quantized precoding for the multiuser massive MIMO downlink. IEEE Transactions on Signal Processing, 65(17):4624-4634, Sep. 2017.

[9] USMAN, O. B.; JEDDA, H.; MEZGHANI, A. ; NOSSEK, J. A.. MMSE precoder for massive MIMO using 1-bit quantization. In: IEEE INTERNATIONAL CONFERENCE ON ACOUSTICS, SPEECH AND SIGNAL PROCESSING (ICASSP), p. 3381-3385, Mar. 2016. 
[10] LI, Y.; TAO, C.; SECO-GRANADOS, G.; MEZGHANI, A.; SWINDLEHURST, A. L. ; LIU, L.. Channel estimation and performance analysis of one-bit massive MIMO systems. IEEE Transactions on Signal Processing, 65(15):4075-4089, Aug. 2017.

[11] STÖCKLE, C.; MUNIR, J.; MEZGHANI, A. ; NOSSEK, J. A.. Channel estimation in massive MIMO systems using 1-bit quantization. In: IEEE 17TH INTERNATIONAL WORKSHOP ON SIGNAL PROCESSING ADVANCES IN WIRELESS COMMUNICATIONS (SPAWC), p. 1-6, Jul. 2016.

[12] JEON, Y.; LEE, N.; HONG, S. ; HEATH, R. W.. One-bit sphere decoding for uplink massive MIMO systems with one-bit ADCs. IEEE Transactions on Wireless Communications, 17(7):4509-4521, Jul. 2018.

[13] STUDER, C.; DURISI, G.. Quantized massive MU-MIMO-OFDM uplink. IEEE Transactions on Communications, 64(6):2387-2399, Jun. 2016.

[14] MOLLÉN, C.; CHOI, J.; LARSSON, E. G. ; HEATH, R. W.. One-bit ADCs in wideband massive MIMO systems with OFDM transmission. In: IEEE INTERNATIONAL CONFERENCE ON ACOUSTICS, SPEECH AND SIGNAL PROCESSING (ICASSP), p. 3386-3390, Mar. 2016.

[15] MOLLÉN, C.; CHOI, J.; LARSSON, E. G. ; HEATH, R. W.. Uplink performance of wideband massive MIMO with one-bit ADCs. IEEE Transactions on Wireless Communications, 16(1):87-100, Jan. 2017.

[16] JACOBSSON, S.; DURISI, G.; COLDREY, M. ; STUDER, C.. Massive MU-MIMO-OFDM downlink with one-bit DACs and linear precoding. In: IEEE GLOBAL COMMUNICATIONS CONFERENCE, Dec. 2017.

[17] ZHANG, J.; DAI, L.; LI, X.; LIU, Y. ; HANZO, L.. On low-resolution ADCs in practical 5G millimeter-wave massive MIMO systems. IEEE Communications Magazine, 56(7):205-211, Jul. 2018.

[18] MO, J.; SCHNITER, P.; PRELCIC, N. G. ; HEATH, R. W.. Channel estimation in millimeter wave MIMO systems with one-bit quantization. In: 48TH ASILOMAR CONFERENCE ON SIGNALS, SYSTEMS AND COMPUTERS, p. 957-961, Nov. 2014. 
[19] MO, J.; HEATH, R. W.. High SNR capacity of millimeter wave MIMO systems with one-bit quantization. In: INFORMATION THEORY AND APPLICATIONS WORKSHOP (ITA), Feb. 2014.

[20] DE LAMARE, R. C.. Massive MIMO systems: Signal processing challenges and future trends. URSI Radio Science Bulletin, 2013(347):8-20, Dec. 2013.

[21] TSE, D.; VISWANATH, P.. Fundamentals of wireless communication. Cambridge university press, 2005.

[22] FLORDELIS, J.; RUSEK, F.; TUFVESSON, F.; LARSSON, E. G. ; EDFORS, O.. Massive MIMO performance-TDD versus FDD: What do measurements say? IEEE Transactions on Wireless Communications, 17(4):2247-2261, Apr. 2018.

[23] VISHWANATH, S.; JINDAL, N. ; GOLDSMITH, A.. Duality, achievable rates, and sum-rate capacity of gaussian MIMO broadcast channels. IEEE Transactions on Information Theory, 49(10):2658-2668, Oct. 2003.

[24] GOLDSMITH, A.; JAFAR, S. A.; JINDAL, N. ; VISHWANATH, S.. Capacity limits of MIMO channels. IEEE Journal on Selected Areas in Communications, 21(5):684-702, Jun. 2003.

[25] VAN DE BEEK, J. .; EDFORS, O.; SANDELL, M.; WILSON, S. K. ; BORJESSON, P. O.. On channel estimation in OFDM systems. In: IEEE 45TH VEHICULAR TECHNOLOGY CONFERENCE. COUNTDOWN TO THE WIRELESS TWENTY-FIRST CENTURY, volumen 2, p. 815-819, 1995.

[26] MOLISCH, A.. Wireless Communications. Wiley - IEEE. Wiley, 2010.

[27] YANG, S.; HANZO, L.. Fifty years of MIMO detection: The road to large-scale MIMOs. IEEE Communications Surveys Tutorials, 17(4):1941-1988, 2015.

[28] WALDEN, R. H.. Analog-to-digital converter survey and analysis. IEEE Journal on Selected Areas in Communications, 17(4):539-550, Apr. 1999.

[29] LE, B.; RONDEAU, T.; REED, J. ; BOSTIAN, C.. Analog-to-digital converters. Signal Processing Magazine, IEEE, 22:69 - 77, 122005. 
[30] MURMANN, B.. ADC performance survey 1997-2020. 2014.

[31] OPPEnhEIM, A.; SCHAFER, R.. Discrete-Time Signal Processing (3rd Edition). Pearson, Aug. 2009.

[32] BUSSGANG, J. J.. Crosscorrelation functions of amplitudedistorted Gaussian signals. Res. Lab. Electron., Massachusetts Inst. Technol., (216):14, Mar. 1952.

[33] JACOVITTI, G.; NERI, A.. Estimation of the autocorrelation function of complex Gaussian stationary processes by amplitude clipped signals. IEEE Transactions on Information Theory, 40(1):239-245, Jan. 1994.

[34] SINGH, J.; PONNURU, S. ; MADHOW, U.. Multi-gigabit communication: the ADC bottleneck1. In: 2009 IEEE INTERNATIONAL CONFERENCE ON ULTRA-WIDEBAND, p. 22-27, Sep. 2009.

[35] WÜBBEN, D.; BOHNKE, R.; KÜHN, V. ; KAMMEYER, K. .. MMSE extension of V-BLAST based on sorted QR decomposition. In: IEEE 58TH VEHICULAR TECHNOLOGY CONFERENCE. VTC 2003-FALL, volumen 1, p. 508-512, 2003.

[36] W. K. WAI; C. Y. TSUI ; CHENG, R. S.. A low complexity architecture of the V-BLAST system. In: IEEE WIRELESS COMMUNICATIONS AND NETWORKING CONFERENCE. CONFERENCE RECORD, volumen 1, p. 310-314, 2000.

[37] BOHNKE, R.; WÜBBEN, D.; KÜHN, V. ; KAMMEYER, K. .. Reduced complexity MMSE detection for BLAST architectures. In: GLOBECOM '03. IEEE GLOBAL TELECOMMUNICATIONS CONFERENCE, volumen 4, p. 2258-2262, 2003.

[38] HASSIBI, B.. An efficient square-root algorithm for BLAST. In: IEEE INTERNATIONAL CONFERENCE ON ACOUSTICS, SPEECH, AND SIGNAL PROCESSING. PROCEEDINGS, volumen 2, p. II737-II740, 2000.

[39] WOLNIANSKY, P. W.; FOSCHINI, G. J.; GOLDEN, G. D. ; VALENZUELA, R. A.. V-BLAST: an architecture for realizing very high data rates over the rich-scattering wireless channel. In: URSI INTERNATIONAL SYMPOSIUM ON SIGNALS, SYSTEMS, AND ELECTRONICS. CONFERENCE PROCEEDINGS, p. 295-300, 1998. 
[40] ChIN, W. H.; COnStantinides, A. G. ; WARD, D. B.. Parallel multistage detection for multiple antenna wireless systems. Electronics Letters, 38(12):597-599, Jun. 2002.

[41] DE LAMARE, R. C.; SAMPAIO-NETO, R.. Minimum mean-squared error iterative successive parallel arbitrated decision feedback detectors for DS-CDMA systems. IEEE Transactions on Communications, 56(5):778-789, May 2008.

[42] VITERBO, E.; BOUTROS, J.. A universal lattice code decoder for fading channels. IEEE Transactions on Information Theory, 45(5):16391642, Jul. 1999.

[43] H. YAO; WORNELL, G. W.. Lattice-reduction-aided detectors for MIMO communication systems. In: IEEE GLOBAL TELECOMMUNICATIONS CONFERENCE, GLOBECOM., volumen 1, p. 424-428, 2002.

[44] WÜBBEN, D.; BOHNKE, R.; KÜHN, V. ; KAMMEYER, K. .. Nearmaximum-likelihood detection of MIMO systems using MMSEbased lattice- reduction. In: 2004 IEEE INTERNATIONAL CONFERENCE ON COMMUNICATIONS, volumen 2, p. 798-802, 2004.

[45] FRICKE, J. C.; SANDELL, M.; MIETZNER, J. ; HOEHER, P. A.. Impact of the Gaussian approximation on the performance of the probabilistic data association MIMO decoder. EURASIP Journal on Wireless Communications and Networking, 2005(5):145035, Dec. 2005.

[46] P. TAN; RASMUSSEN, L. K.. The application of semidefinite programming for detection in CDMA. IEEE Journal on Selected Areas in Communications, 19(8):1442-1449, Aug. 2001.

[47] JOHAM, M.; UTSCHICK, W. ; NOSSEK, J. A.. Linear transmit processing in MIMO communications systems. IEEE Transactions on Signal Processing, 53(8):2700-2712, Aug. 2005.

[48] WEINGARTEN, H.; STEINBERG, Y. ; SHAMAI, S. S.. The capacity region of the gaussian multiple-input multiple-output broadcast channel. IEEE Transactions on Information Theory, 52(9):3936-3964, Sep. 2006.

[49] TOMLINSON, M.. New automatic equaliser employing modulo arithmetic. Electronics Letters, 7(5):138-139, Mar. 1971. 
[50] MULLER, R. R.; GUO, D. ; MOUSTAKAS, A. L.. Vector precoding for wireless MIMO systems and its replica analysis. IEEE Journal on Selected Areas in Communications, 26(3):530-540, Apr. 2008.

[51] ZU, K.; DE LAMARE, R. C. ; HAARDT, M.. Multi-branch TomlinsonHarashima precoding design for MU-MIMO systems: Theory and algorithms. IEEE Transactions on Communications, 62(3):939-951, Mar. 2014.

[52] XIONG, Y.; ZHANG, Z.; WEI, N.; LI, B. ; CHEN, Y.. Performance analysis of uplink massive MIMO systems with variable-resolution ADCs using MMSE and MRC detection. Transactions on Emerging Telecommunications Technologies, 30(5):3549, Jan. 2019.

[53] STEIN, M. S.; BAR, S.; NOSSEK, J. A. ; TABRIKIAN, J.. Performance analysis for channel estimation with 1-bit adc and unknown quantization threshold. IEEE Transactions on Signal Processing, 66(10):2557-2571, May 2018.

[54] C. RISI, D. P.; LARSSON, E. G.. Massive MIMO with 1-bit ADC. https://arxiv.org/abs/1404.7736, 2014.

[55] CHOI, J.; MO, J. ; HEATH, R. W.. Near maximum-likelihood detector and channel estimator for uplink multiuser massive MIMO systems with one-bit ADCs. IEEE Transactions on Communications, 64(5):2005-2018, May 2016.

[56] ALEXANDER, T. S.. Adaptive signal processing: theory and applications. Springer Science \& Business Media, 2012.

[57] DE LAMARE, R. C.. Adaptive and iterative multi-branch MMSE decision feedback detection algorithms for multi-antenna systems. IEEE Transactions on Wireless Communications, 12(10):5294-5308, Oct. 2013.

[58] WANG, X.; POOR, H. V.. Iterative (turbo) soft interference cancellation and decoding for coded CDMA. IEEE Transactions on Communications, 47(7):1046-1061, Jul. 1999.

[59] UCHOA, A. G. D.; HEALY, C. T. ; DE LAMARE, R. C.. Iterative detection and decoding algorithms for MIMO systems in blockfading channels using LDPC codes. IEEE Transactions on Vehicular Technology, 65(4):2735-2741, Apr. 2016. 
[60] HU, X.; ElefTheriOU, E.; ARNOLD, D. M. ; DHOLAKIA, A.. Efficient implementations of the sum-product algorithm for decoding LDPC codes. In: IEEE GLOBAL TELECOMMUNICATIONS CONFERENCE, volumen 2, p. 1036-1036E vol.2, San Antonio, TX, USA, Nov. 2001.

[61] ZHANG, X.; SIEGEL, P. H.. Quantized iterative message passing decoders with low error floor for LDPC codes. IEEE Transactions on Communications, 62(1):1-14, Jan- 2014.

[62] ALVARADO, A.; NUNEZ, V.; SZCZECINSKI, L. ; AGRELL, E.. Correcting suboptimal metrics in iterative decoders. In: IEEE INTERNATIONAL CONFERENCE ON COMMUNICATIONS, p. 1-6, Dresden, Germany, Jun. 2009.

[63] GALLAGER, R.. Low-density parity-check codes. IRE Transactions on Information Theory, 8(1):21-28, 1962.

[64] GILBERT, E. N.. Increased information rate by oversampling. IEEE Transactions on Information Theory, 39(6):1973-1976, Nov. 1993.

[65] $\mathrm{KOCH}, \mathrm{T}$.; LAPIDOTH, A.. Increased capacity per unit-cost by oversampling. In: IEEE 26TH CONVENTION OF ELECTRICAL AND ELECTRONICS ENGINEERS IN ISRAEL, p. 684-688, Nov. 2010.

[66] HÄLSIG, T.; LANDAU, L. ; FETTWEIS, G.. Information rates for faster-than-Nyquist signaling with 1-bit quantization and oversampling at the receiver. In: IEEE 79TH VEHICULAR TECHNOLOGY CONFERENCE (VTC SPRING), May 2014.

[67] ÜÇÜNCÜ, A. B.; YILMAZ, A. O.. Oversampling in one-bit quantized massive MIMO systems and performance analysis. IEEE Transactions on Wireless Communications, 17(12):7952-7964, Dec. 2018.

[68] SHAO, Z.; LANDAU, L. T. N. ; DE LAMARE, R. C.. Channel estimation using 1-bit quantization and oversampling for large-scale multiple-antenna systems. In: IEEE INTERNATIONAL CONFERENCE ON ACOUSTICS, SPEECH AND SIGNAL PROCESSING (ICASSP), p. 46694673, May 2019.

[69] SHAO, Z.; LANDAU, L. T. N. ; DE LAMARE, R. C.. Sliding window based linear signal detection using 1-bit quantization and oversampling for large-scale multiple-antenna systems. In: IEEE STA- 
TISTICAL SIGNAL PROCESSING WORKSHOP (SSP), p. 183-187, Jun. 2018.

[70] COLERI, S.; ERGEN, M.; PURI, A. ; BAHAI, A.. Channel estimation techniques based on pilot arrangement in OFDM systems. IEEE Transactions on Broadcasting, 48(3):223-229, Sep. 2002.

[71] SCHLÜTER, M.; DÖRPINGHAUS, M. ; FETTWEIS, G. P.. Bounds on channel parameter estimation with 1-bit quantization and oversampling. In: IEEE 19TH INTERNATIONAL WORKSHOP ON SIGNAL PROCESSING ADVANCES IN WIRELESS COMMUNICATIONS (SPAWC), p. 1-5, Jun. 2018.

[72] STEIN, M.; MEZGHANI, A. ; NOSSEK, J. A.. A lower bound for the fisher information measure. IEEE Signal Processing Letters, 21(7):796799, Jul. 2014.

[73] H. L. VAN TREES, K. L. B.; TIAN, Z.. Detection Estimation and Modulation Theory, Part I: Detection, Estimation, and Filtering Theory, Detection Estimation and Modulation Theory. Wiley, 2013.

[74] STEIN, M. S.. Performance analysis for time-of-arrival estimation with oversampled low-complexity 1-bit a/d conversion. In: IEEE INTERNATIONAL CONFERENCE ON ACOUSTICS, SPEECH AND SIGNAL PROCESSING (ICASSP), p. 4491-4495, Mar. 2017.

[75] CLERCKX, B.; KIM, G. ; KIM, S.. Correlated fading in broadcast MIMO channels: Curse or blessing? In: IEEE GLOBAL TELECOMMUNICATIONS CONFERENCE, Nov. 2008.

[76] SHAO, Z.; LANDAU, L. T. N. ; DE LAMARE, R. C.. Channel estimation for large-scale multiple-antenna systems using 1-bit ADCs and oversampling. IEEE Access, 8:85243-85256, 2020.

[77] MEZGHANI, A.; NOSSEK, J. A.. Capacity lower bound of MIMO channels with output quantization and correlated noise. In: PROCEEDINGS IEEE INTERNATIONAL SYMPOSIUM INFORMATION THEORY, Boston, USA, Jul. 2012.

[78] DE LAMARE, R. C.; SAMPAIO-NETO, R.. Adaptive reduced-rank processing based on joint and iterative interpolation, decimation, and filtering. IEEE Transactions on Signal Processing, 57(7):2503-2514, Jul. 2009. 
[79] BOYD, S.; VANDENBERGHE, L.. Convex optimization. Cambridge university press, 2004.

[80] WANG, H.; YAN, S.; XU, D.; TANG, X. ; HUANG, T.. Trace ratio vs. ratio trace for dimensionality reduction. In: IEEE CONFERENCE ON COMPUTER VISION AND PATTERN RECOGNITION, Jun. 2007.

[81] JIA, Y.; NIE, F. ; ZHANG, C.. Trace ratio problem revisited. IEEE Transactions on Neural Networks, 20(4):729-735, Apr. 2009.

[82] GHOJOGH, B.; KARRAY, F. ; CROWLEY, M.. Eigenvalue and generalized eigenvalue problems: Tutorial, 2019.

[83] DEVYVER, P.; KITTLER, J.. Pattern Recognition: A Statistical Approach. Prentice-Hall, 1982.

[84] ÜÇÜNCÜ, A. B.; YILMAZ, A. O.. Performance analysis of faster than symbol rate sampling in 1-bit massive MIMO systems. In: IEEE INTERNATIONAL CONFERENCE ON COMMUNICATIONS (ICC), p. 1-6, Paris, May 2017.

[85] TREFETHEN, L.; BAU, D.. Numerical Linear Algebra. SIAM: Society for Industrial and Applied Mathematics, Apr 1997. 
A

\section{Proof of the linear transformation in (3-3)}

The Bussgang theorem in (2-32) gives us an insight about the calculation of cross-correlation of a Gaussian signal before and after the nonlinear operation. Assume that $\mathbf{y}_{Q}$ in (3-2) can be reformulated as the following linear form

$$
\mathbf{y}_{Q}=\mathbf{A y}+\mathbf{n}_{q},
$$

where $\mathbf{A}$ is the linear operator and $\mathbf{n}_{q}$ is the quantization error. The crosscorrelation of the input and output of the quantizer is given by

$$
\mathbf{C}_{\mathbf{y}_{Q} \mathbf{y}}=E\left\{\mathbf{y}_{Q} \mathbf{y}^{H}\right\}=E\left\{\left(\mathbf{A y}+\mathbf{n}_{q}\right) \mathbf{y}^{H}\right\}=\mathbf{A C}_{\mathbf{y}}
$$

where we have assumed $\mathbf{n}_{q}$ is independent of $\mathbf{y}$ and the resulting crosscorrelation $E\left\{\mathbf{n}_{q} \mathbf{y}^{H}\right\}$ is zero. According to (2-32) we obtain

$$
\mathbf{A}=\sqrt{\frac{2}{\pi}} \operatorname{diag}\left(\mathbf{C}_{\mathbf{y}}\right)^{-\frac{1}{2}} .
$$

The auto-correlation of $\mathbf{y}_{Q}$ is calculated as

$$
\mathbf{C}_{\mathbf{y}_{Q}}=E\left\{\mathbf{y}_{Q} \mathbf{y}_{Q}^{H}\right\}=\mathbf{A C}_{\mathbf{y}} \mathbf{A}+\mathbf{C}_{\mathbf{n}_{q}}
$$

and the resulting $\mathbf{C}_{\mathbf{n}_{q}}$ is

$$
\mathbf{C}_{\mathbf{n}_{q}}=E\left\{\mathbf{n}_{q} \mathbf{n}_{q}^{H}\right\}=\mathbf{C}_{\mathbf{y}_{Q}}-\mathbf{A} \mathbf{C}_{\mathbf{y}} \mathbf{A} .
$$




\section{B}

\section{Proof of (4-22)}

Recall the optimization problem

$$
\mathbf{W}_{\mathrm{LMMSE}}=\underset{\mathbf{W}}{\arg \min } E\left\{\left\|\mathbf{h}^{\prime}-\mathbf{W}_{\mathbf{Q}_{p}}\right\|^{2}\right\} .
$$

Taking the partial derivative with respect to $\mathbf{W}^{H}$, we obtain

$$
\frac{\partial E\left\{\left\|\mathbf{h}^{\prime}-\mathbf{W}_{\mathcal{Q}_{p}}\right\|^{2}\right\}}{\partial \mathbf{W}^{H}}=-E\left\{\mathbf{h}^{\prime} \mathbf{y}_{\mathcal{Q}_{p}}^{H}\right\}+\mathbf{W} E\left\{\mathbf{y}_{\mathcal{Q}_{p}} \mathbf{y}_{\mathcal{Q}_{p}}^{H}\right\} .
$$

Inserting (4-14) into (B-2), the LMMSE filter becomes

$$
\begin{aligned}
\mathbf{W}_{\text {LMMSE }} & =E\left\{\mathbf{h}^{\prime} \mathbf{y}_{\mathcal{Q}_{p}}^{H}\right\} E\left\{\mathbf{y}_{\mathcal{Q}_{p}} \mathbf{y}_{\mathcal{Q}_{p}}^{H}\right\}^{-1} \\
& =\left(E\left\{\mathbf{h}^{\prime} \mathbf{h}^{\prime H}\right\} \tilde{\boldsymbol{\Phi}}_{p}^{H}+E\left\{\mathbf{h}^{\prime} \tilde{\mathbf{n}}_{p}^{H}\right\}\right) \mathbf{C}_{\mathbf{y}_{\mathcal{Q}_{p}}^{-1}} .
\end{aligned}
$$

Since $\mathbf{h}^{\prime}$ is uncorrelated with $\mathbf{n}_{p}$ and $\mathbf{n}_{q}[10]$, we have

$$
E\left\{\mathbf{h}^{\prime} \tilde{\mathbf{n}}_{p}^{H}\right\}=E\left\{\mathbf{h}^{\prime}\left(\mathbf{A}_{p} \mathbf{n}_{p}+\mathbf{n}_{q}\right)^{H}\right\}=\mathbf{0} .
$$

The resulting LRA-LMMSE channel estimator is given by

$$
\hat{\mathbf{h}}_{\text {LRA-LMMSE }}^{\prime}=\mathbf{R}_{\mathbf{h}^{\prime}} \tilde{\boldsymbol{\Phi}}_{p}^{H} \mathbf{C}_{\mathbf{y}_{\mathcal{Q}_{p}}^{-1}}^{-\mathbf{y}_{\mathcal{Q}_{p}}}
$$




\section{C \\ Proof of (4-28)}

Defining $\epsilon(n)=\overline{\mathbf{h}}^{n_{r}}(n)-\mathbf{h}^{\prime n_{r}}$ and inserting it into (4-27), we obtain

$$
\begin{aligned}
\epsilon(n+1) & =\epsilon(n)+\mu \tilde{\boldsymbol{\Phi}}_{p}^{n_{r}}(n)^{H}\left(\mathbf{y}_{\mathcal{Q}_{p}}^{n_{r}}(n)-\tilde{\boldsymbol{\Phi}}_{p}^{n_{r}}(n) \overline{\mathbf{h}}^{n_{r}}(n)\right) \\
& =\epsilon(n)+\mu \tilde{\boldsymbol{\Phi}}_{p}^{n_{r}}(n)^{H} \mathbf{y}_{\mathcal{Q}_{p}}^{n_{r}}(n)-\mu \tilde{\boldsymbol{\Phi}}_{p}^{n_{r}}(n)^{H} \tilde{\boldsymbol{\Phi}}_{p}^{n_{r}}(n)\left(\epsilon(n)+\mathbf{h}^{\prime n_{r}}\right) \\
& =\left(\mathbf{I}-\mu \tilde{\boldsymbol{\Phi}}_{p}^{n_{r}}(n)^{H} \tilde{\boldsymbol{\Phi}}_{p}^{n_{r}}(n)\right) \epsilon(n)+\mu \tilde{\boldsymbol{\Phi}}_{p}^{n_{r}}(n)^{H}\left(\mathbf{y}_{\mathcal{Q}_{p}}^{n_{r}}(n)-\tilde{\boldsymbol{\Phi}}_{p}^{n_{r}}(n) \mathbf{h}^{\prime_{r}}\right) .
\end{aligned}
$$

Taking the expected value from $\epsilon(n+1)$, we have

$$
E\{\epsilon(n+1)\}=\left(\mathbf{I}-\mu E\left\{\tilde{\boldsymbol{\Phi}}_{p}^{n_{r}}(n)^{H} \tilde{\mathbf{\Phi}}_{p}^{n_{r}}(n)\right\}\right) E\{\epsilon(n)\} .
$$

With the eigenvalue decomposition $E\left\{\tilde{\boldsymbol{\Phi}}_{p}^{n_{r}}(n)^{H} \tilde{\mathbf{\Phi}}_{p}^{n_{r}}(n)\right\}=\mathbf{Q} \Gamma \mathbf{Q}^{H},(\mathrm{C}-2)$ can be written as

$$
\begin{aligned}
\mathbf{Q}^{H} E\{\epsilon(n+1)\} & =\mathbf{Q}^{H}\left(\mathbf{I}-\mu \mathbf{Q} \boldsymbol{\Gamma} \mathbf{Q}^{H}\right) E\{\epsilon(n)\} \\
& =(\mathbf{I}-\mu \boldsymbol{\Gamma}) \mathbf{Q}^{H} E\{\epsilon(n)\},
\end{aligned}
$$

where $\mathbf{Q}$ is a unitary matrix and $\boldsymbol{\Gamma}$ is a diagonal matrix, whose diagonal entries are the eigenvalues of $E\left\{\tilde{\boldsymbol{\Phi}}_{p}^{n_{r}}(n)^{H} \tilde{\boldsymbol{\Phi}}_{p}^{n_{r}}(n)\right\}$. With $\mathbf{u}(n)=\mathbf{Q}^{H} E\{\epsilon(n)\}$, (C-3) is then

$$
\mathbf{u}(n+1)=(\mathbf{I}-\mu \boldsymbol{\Gamma}) \mathbf{u}(n) .
$$

Decoupling the matrix form into individual elements we get

$$
\begin{aligned}
u_{n_{t}}(n+1) & =\left(1-\mu \gamma_{n_{t}}\right) u_{n_{t}}(n) \\
& =\left(1-\mu \gamma_{n_{t}}\right)^{\tau-l_{\mathrm{win}}+1} u_{n_{t}}(1), \quad n_{t}=1, \ldots, N_{t} .
\end{aligned}
$$

In order for the LRA-LMS to converge, we must have

$$
\left|1-\mu \gamma_{n_{t}}\right|<1
$$

The stability condition is then given by

$$
0<\mu<\frac{2}{\gamma_{\max }}
$$

where $\gamma_{\max }$ is the largest eigenvalue of $E\left\{\tilde{\boldsymbol{\Phi}}_{p}^{n_{r}}(n)^{H} \tilde{\boldsymbol{\Phi}}_{p}^{n_{r}}(n)\right\}$. 
D

\section{Proof of Lemma 1}

The bias of the adaptive estimator $\hat{\mathbf{R}}_{\mathbf{h}^{\prime}}$ is firstly examined. The expected value of $\hat{\mathbf{h}}^{\prime}(n)$ in $(4-31)$ is

$$
E\left\{\hat{\mathbf{h}}^{\prime}(n)\right\}=E\left\{\left(\mathbf{x}_{p}^{\prime T}(n) \otimes \mathbf{I}_{N_{r}} \otimes \mathbf{Z}^{\prime} \mathbf{u}\right)^{+} \mathbf{y}_{\mathcal{Q}}(n)\right\} .
$$

From the Bussgang theorem (4-30) can be decomposed as

$$
\begin{aligned}
\mathbf{y}_{\mathcal{Q}}(n) & =\mathcal{Q}\left(\left(\mathbf{x}_{p}^{\prime T}(n) \otimes \mathbf{I}_{N_{r}} \otimes \mathbf{Z}^{\prime} \mathbf{u}\right) \mathbf{h}^{\prime}+\mathbf{n}(n)\right) \\
& =\mathbf{A}_{p}^{\prime}(n)\left(\left(\mathbf{x}_{p}^{\prime T}(n) \otimes \mathbf{I}_{N_{r}} \otimes \mathbf{Z}^{\prime} \mathbf{u}\right) \mathbf{h}^{\prime}+\mathbf{n}(n)\right)+\mathbf{n}_{q}(n),
\end{aligned}
$$

where $\mathbf{A}_{p}^{\prime}(n)$ is the linear operator and $\mathbf{n}_{q}(n)$ is the statistically equivalent quantizer noise. Substituting (D-2) into (D-1) and with $\boldsymbol{\Phi}^{\prime}(n)=\left(\mathbf{x}^{T}(n) \otimes\right.$ $\left.\mathbf{I}_{N_{r}} \otimes \mathbf{Z}^{\prime} \mathbf{u}\right)$, we obtain

$$
\begin{aligned}
E\left\{\hat{\mathbf{h}}^{\prime}(n)\right\} & =E\left\{\boldsymbol{\Phi}^{\prime}(n)^{+}\left(\mathbf{A}_{p}^{\prime}(n)\left(\boldsymbol{\Phi}^{\prime}(n) \mathbf{h}^{\prime}+\mathbf{n}(n)\right)+\mathbf{n}_{q}(n)\right)\right\} \\
& =E\left\{\boldsymbol{\Phi}^{\prime}(n)^{+} \mathbf{A}_{p}^{\prime}(n) \boldsymbol{\Phi}^{\prime}(n) \mathbf{h}^{\prime}\right\}+E\left\{\boldsymbol{\Phi}^{\prime}(n)^{+} \mathbf{A}_{p}^{\prime}(n) \mathbf{n}(n)\right\}+E\left\{\boldsymbol{\Phi}^{\prime}(n)^{+} \mathbf{n}_{q}(n)\right\} .
\end{aligned}
$$

Since $\boldsymbol{\Phi}^{\prime}(n)$ and $\mathbf{n}(n)$ are uncorrelated and $E\{\mathbf{n}(n)\}=\mathbf{0}$, we have

$$
E\left\{\boldsymbol{\Phi}^{\prime}(n)^{+} \mathbf{A}_{p}^{\prime}(n) \mathbf{n}(n)\right\}=\mathbf{0} .
$$

Similarly,

$$
E\left\{\boldsymbol{\Phi}^{\prime}(n)^{+} \mathbf{n}_{q}(n)\right\}=\mathbf{0} .
$$

Equation (D-3) can be further simplified as

$$
E\left\{\hat{\mathbf{h}}^{\prime}(n)\right\}=E\left\{\boldsymbol{\Phi}^{\prime}(n)^{+} \mathbf{A}_{p}^{\prime}(n) \boldsymbol{\Phi}^{\prime}(n)\right\} \mathbf{h}^{\prime} .
$$

The matrix $\mathbf{A}_{p}^{\prime}(n)$ depends on $\mathbf{R}_{\mathbf{h}^{\prime}}$ such that the expectation in (D-6) can be different from the identity matrix especially for channels without normalization, which verifies that (4-31) has an unknown bias [73]. With the analysis above, it is concluded that the adaptive estimator $\hat{\mathbf{R}}_{\mathbf{h}^{\prime}}$ is also biased, which shows that the estimation procedures together with the proposed LRA channel estimators are biased. 